\title{
Flora of kurgans in the forest steppe zone in Ukraine
}

\author{
BARBARA SUDNIK-WÓJCIKOWSKA \\ IVAN IVANOVYCH MOYSIYENKO
}

СуднІК-ВойциКОВСьКА Б., МойсІєнКО І.І., 2010: Флора курганів лісостепової зони України. Чорноморськ. бот. ж., Т. 6, № 2: 162-199.

Представлені результати вивчення флористичного різноманіття флори курганів лісостепової зони проведені на території Кіровоградської, Полтавської та Черкаської областей. Всього досліджено 25 курганів понад 3 м висотою на площі 12092,4 км². Досліджена флора курганів Лісостепу виявилась багатшою на види порівняно 33 смугами степовою зони і налічуе 460 видів судинних рослин. Кількість видів на одному кургані варіюе від 85 до 159, а в середньому становить 107,5. Як і в Понтичному різнотравно- та багаторізнотравнозлаковому степу у флорі курганів лісостепу переважають гемікриптофіти $(39,4 \%)$, тоді як в південніших степових смугах на курганах домінують терофіти. Також більш чисельними виявились фанерофіти (10,0\%). Короткоживучі (одно-, дво- трирічні) види складають $33,1 \%$ флори курганів лісостепової зони. Більшість 3 них належить до синантропних видів, зокрема є бур'янами, що проникли 3 оточуючих полів. В цілому на курганах в лісостепу виявлено 112 антропофітів. Археофіти та кенофіти складають $13,7 \%$ та 7,6\% флори курганів, відповідно.

Загалом на курганах представлені види, що мають оптимум трапляння у складі 20 синтаксонів вищого рангу. Види асоційовані з степовими синтаксонами (FestucoBrometea, Festucetea vaginatae, Polygono-Artemisietea та Galietalia veri) складають 49,3\% дослідженої флори. Як і у флорі курганів справжніх та пустельних степів в лісостепу переважають види класів Festuco-Brometea та Stellarietea mediae, що вказує на напівнатуральний характер флори курганів. Також у флорі курганів лісостепу представлені види 5 класів дерев'янистої та чагарникової рослинності: QuercoFagetea, Urtico-Sambucetea, Robinietea та Salicetea purpureae.

Загалом на курганах у флорі лісостепової зони виявлено 47 раритетних видів. Дослідження проведені в лісостеповій зоні підтверджують, що вони є рефугіумом степової флори. Вони можуть відігравати значну роль у відновлені природного рослинного покриву. Тому, кургани повинні охоронятися і як пам'ятники археології, і як пам'ятки природи.

Ключові слова: кургани, рефугіум степової флори, флористичне різноманіття, лісостеп, охорона курганів, Полтавська, Черкаська та Кіровоградська області, Украӥна

SudNiK-WóJCikowsKa B. MoysiyenKo I.I., 2010: Flora of kurgans in the forest steppe zone in Ukraine. Chornomors'k. bot. z., Vol. 6, № 2: 162-199.

The results of study of floristic diversity of kurgans in the forest steppe zone in Poltava, Cherkasy and Kirovograd Regions, are presented. Twenty-five kurgans of more than $3 \mathrm{~m}$ high distributed over an area approx. 12092,4 $\mathrm{km}^{2}$, were surveyed. Being estimated at 460 species, the investigated kurgan flora contains more species than the flora of the barrows in each of the 3 steppe zones. The number of species on a single kurgan varied from 85 to 159, with 107,5 in average. As in the Pontic herb(-rich) grass steppe zone, hemicryptophytes dominated in the flora of the kurgans $(39,4 \%)$ in the forest steppe zone, whereas therophytes prevailed in the barrow flora in the steppe zones southwards. Phanerophytes were more numerous as well $(10,0 \%)$. Short-living plants (one, two or three years old) constituted $33,1 \%$ of the kurgan flora in the forest steppe zone. Most of them are synanthropic species, e.g. weeds from the surrounding fields. The total number of alien species (anthropophytes) reached 112. Archaeophytes and kenophytes comprised 13,7\% and $7,6 \%$ of the kurgan flora.

A total of 20 syntaxa of a higher rank were represented in all the kurgans studied. Species associated with steppe syntaxa such as: Festuco-Brometea, Festucetea vaginatae, 
Polygono-Artemisietea and Galietalia veri comprised as much as $49,3 \%$ of the kurgan flora. As in the desert and grass steppes, species belonging to classes Festuco-Brometea and Stellarietea mediae were predominant in the forest steppe as well, which also confirmed the semi-natural character of the kurgan flora. A total of 5 classes of tree and shrub communities were represented on the kurgans in the forest steppe zone: QuercoFagetea, Urtico-Sambucetea, Robinietea and Salicetea purpureae.

The number of species of particularly high floristic value was estimated at 47 . The investigations carried out in the forest steppe zone confirm that kurgans constitute refugia for the steppe flora. They could play an important role in the local restoration of the natural plant cover. Therefore, they should be put under protection both as archeological and nature monuments.

Key words: barrows, refugia of steppe flora, floristic diversity, forest steppe, protection of kurgans, Poltava, Cherkasy, Kirovograd Region, Ukraine

СудНІК-ВоЙЦИКОВСКАЯ Б., МОЙСИЕНКО И.И., 2010: Флора курганов лесостепной зоны Украины. Черноморск. бот. ж., Т. 6, № 2: 162-199.

Представлены результаты изучения флористического разнообразия флоры курганов лесостеповой зоны проведенные на територии Кировоградской, Полтавской и Черкасской областей. Всего исследовано 25 курганов более 3 м высотой на площади

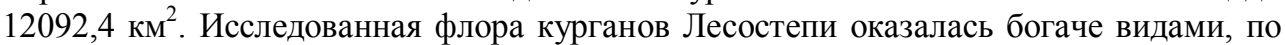
сравнению с 3 полосами степной зоны и насчитывает 460 видов сосудистых растений. Количество видов на одном кургане варирует от 85 до 159, а в среднем систавляет 107,5. Как и в Понтичной разнотравно- и богаторазнотравнозлаковой степи во флоре курганов лесостепи преобладают гемикриптофиты $(39,4 \%)$, тогда как в болем южных степных полосах на курганах доминируют терофиты. Также более многочисленными оказались фанерофиты (10,0\%). Краткоживущие (одно-, двутрехлетние) виды составляют $33,1 \%$ флоры курганов лесостеповой зоны. Большинство из них принадлежит к синантропным видам, в частности сорнякам, которые проникли с окружающих полей. В целом на курганах в лесостепи выявлено 112 антропофитов. Археофіти и кенофіти составляют 13,7\% и 7,6\% флоры курганов, соответственно.

В целом на курганах представлены виды, имеющие оптимум встречаемости в составе 20 синтаксонов высокого ранга. Виды ассоциированные со степными синтаксонами (Festuco-Brometea, Festucetea vaginatae, Polygono-Artemisietea та Galietalia veri) составлябт 49,3\% осследованний флоры. Как и во флоре курганов настояних и пустынных степей в лесостепи преобладают виды класов Festuco-Brometea и Stellarietea mediae, что вказывает на полуестественный характер флоры курганов. Также во флоре курганов лесостепи представлены виды 5 класов древесной и кустарниковой растительности: Querco-Fagetea, Urtico-Sambucetea, Robinietea и Salicetea purpureae.

В целом на курганах во флоре лесостепной зоны выявлено 47 раритетных видов. Исследования проведенные в лесостепной зоне подтверждают, что они являются рефугиумом степной флоры. Они могут играть значительную роль при востановлении природного растительного покрова. Поэтому, курганы должны охранятся и как памятники археологии, и как памятки природы.

Ключевые слова: курганы, рефугиум степной флоры, флористическое разнообразие, лесостепь, охрана курганов, Полтавская, Черкасская и Кировоградская области, Украина.

\section{Introduction}

The present work is the last one in the series of publications dealing with the biodiversity of kurgan flora in the steppe and forest steppe zones of Ukraine. Earlier papers concerned the flora of kurgans located southwards: desert steppe - the west and central Pontic steppe zone [MOYSIYENKO, SUDNIK-WÓJCIKOWSKA 2006], grass steppe zone - ,poor forbs" - the west Pontic grass steppe zone [SUDNIK-WÓJCIKOWSKA, MOYSIYENKO 2006], and "rich forbs" - the Pontic herb(-rich) grass steppe zone [MOYSIYENKO, SUDNIK-WÓJCIKOWSKA 2009].

The aim of this study was to assess the richness and specific characters of the flora of kurgans within the forest steppe zone. 


\section{Study area}

Kurgans investigated in the forest steppe zone are located in Poltava, Cherkasy (Cherkassy) and Kirovograd Regions. The forest steppe zone is an irregular strip that extends from the Balkan Peninsula to Changan Mountain [ЛАВРенко та ін., 1991]. In Ukraine, it is part of the East European forest steppe province and covers about 34\% of the country's territory [МАРИНИЧ, ШИЩЕНКО, 2003]. The above mentioned zone runs approximately evenly with a parallel of latitude and occupies the whole central part of Ukraine. The forest steppe belt is about $1100 \mathrm{~km}$ long and $200-300 \mathrm{~km}$ wide. It covers the Volhynia-Podilia Upland, the Dnieper Upland, the Dnieper Lowland and the western part of the Central Russian Upland. The forest steppe zone is intersected by a number of rivers: Dniester, Dnieper, Pivdenny Bug and Seversky Donets. The density of river network decreases from west to east. Erosional landforms dominate in the landscape, so the river valleys have an asymmetric shape. The zone is dissected by a number of balkas, deeply notched valleys, erosion ravines. Flat watersheds often have suffosion soil falldowns [ГЕОБОТАНІЧНЕ РАЙОНУВАННЯ..., 1977, ДІДУХ, ШЕЛЯГ-СОСОНКО, 2003; МАРИНИЧ, ШИЩЕНКО, 2003].

The forest steppe zone is characterized by a temperate climate with a warm summer and a moderately cold winter. The climate becomes more continental towards the east. The average January temperatures range from 15 to $-5^{0} \mathrm{C}$, while the average July temperatures vary from 18 to $20^{\circ} \mathrm{C}$. The mean annual precipitation is usually $550-750 \mathrm{~mm}$ in the west and $450 \mathrm{~mm}$ in the east, which slightly exceeds evaporation. Humidity is close to optimal. In summer the rain comes in downpours [ПРИРОДА..., 1984].

The main soils of the belt are deep black chernozems, leached black soils, degraded black soils or grey forest soils as well as parabrown soils. Sandy soils as well as solonetz-like black soils, meadow soils in combination with solonetz and solontschak soils are on river terraces, whereas mud and alluvial soils are found within river valleys [ПРИРОДА..., 1986].

The forest steppe zone is a macromosaic of forests, mainly on leached black soils, and meadow steppe on deep black soils. Among forest communities, deciduous forests dominate, especially oak forests with Quercus robur. Other tree species such as: Fagus sylvatica (in the west), Carpinus betulus (in the centre), Acer sp.div. Tilia cordata and Fraxinus excelsior are found alongside the oak. Alluvial forests occur in river valleys on sandy soils. Pine forests as well as Betula and Populus tremula kolka forests grow along river valleys [ГЕОБОТАНІЧНЕ РАЙОНУВАННЯ..., 1977]. According to the nomenclature proposed in publication Map of the natural vegetation of Europe [MAP..., 2000] the investigated kurgans are located in 4 subzones (Fig. 1): F41 - East Polish-Ukrainian lime-pedunculate oak-hornbeam forests; F44 - Podolian-Moldavian thermophilous hornbeam-pedunculate oak forests; L3 - MoldavianUkrainian meadow steppes alternating with hornbeam-pedunculate oak forests in the south with Tatarian maple-pedunculate oak forests; L4 - South Sarmatian meadow steppes alternating with salt vegetation on solonetz soils and pedunculate oak forests.

In these areas the steppe has been ploughed up, destroyed and is now largely under cultivation. The meadow steppe was originally rich in species. Nowadays, remnants of the steppe have been preserved within nature reserves and areas useless for agriculture, e.g. on slopes of river terraces, balkas, ravines, especially with rock outcrops.

The fine-grained deep black soils and leached black soils that formed under the steppe, which is rich in species, are characterized by a high humus content and are one of the most fertile soils in the world. At the same time the forest steppe has been intensively exploited by man for a long time. About $80 \%$ of the steppe is cultivated. The climate and soil conditions are most favourable for the cultivation of wheat, corn, buckwheat and sugar beet.

A total of 25 kurgans (F1-F25) were investigated (Fig. 1). Most of them (18) are located on the right bank of the Dnieper. GPS data - coordinates of barrows are presented in Table 1. 


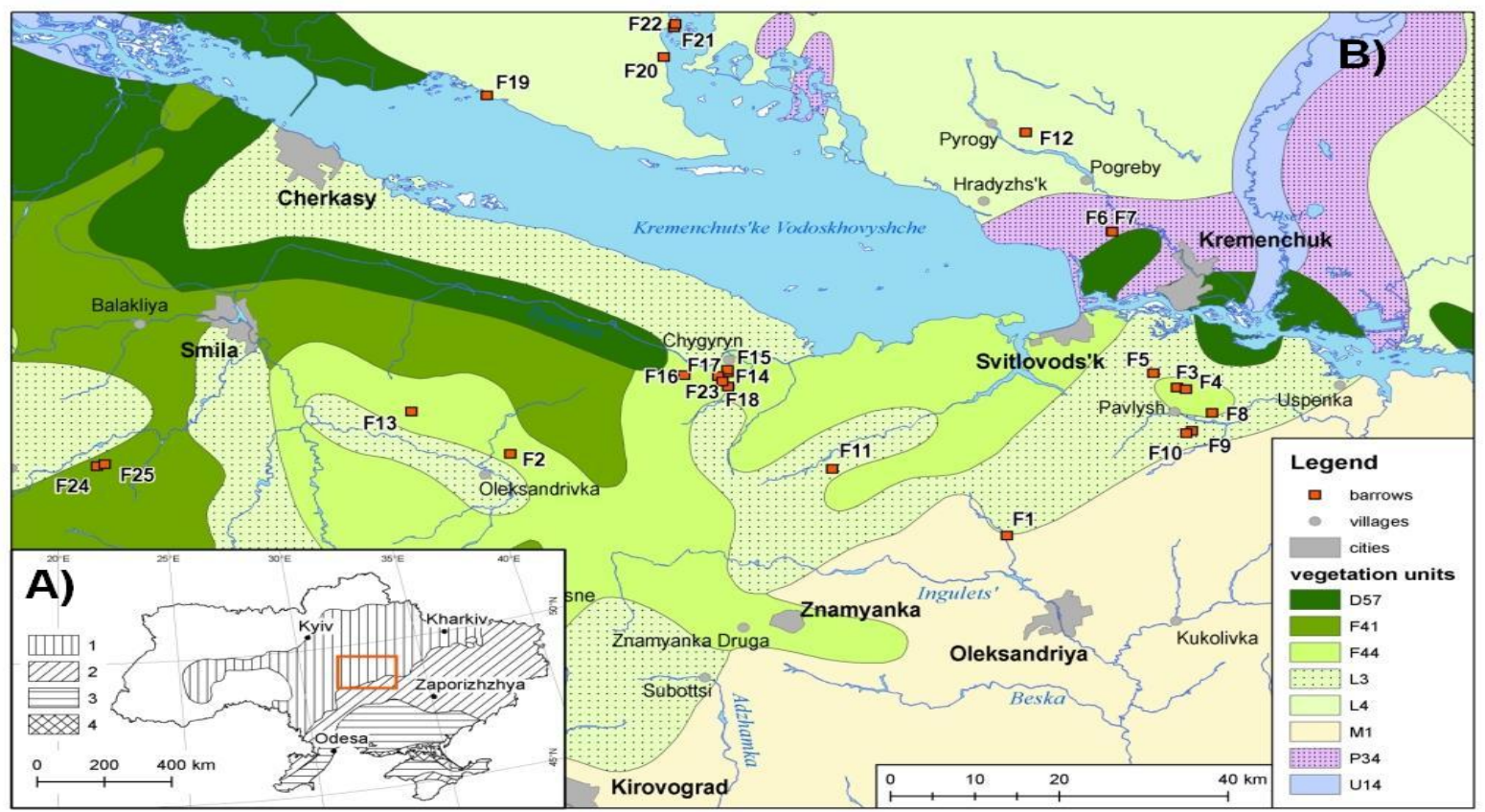

Fig. 1. A) The distribution of the investigated kurgans in the Poltava, Cherkasy and Kirovograd Regions; B) The location of the investigated area and the various types of steppes and forest steppe in Ukraine (according to Map of the natural vegetation of Europe, Bohn et al. 2000): D57 - South European xerophytic herb- and grass-rich pine and oak-pine forests on shallow carbonate soils with steppe plants; F41 - East Polish-Ukrainian lime-pedunculate oakhornbeam forests; F44 - Podolian-Moldavian thermophilous hornbeam-pedunculate oak forests; L3 Moldavian-Ukrainian meadow steppes alternating with hornbeam-pedunculate oak forests in the south with Tatarian maple-pedunculate oak forests; L4 - South Sarmatian meadow steppes alternating with salt vegetation on solonetz soils and pedunculate oak forests; M1 - west and central Pontic herb-rich grass steppe; P34 - West and East Pontic salt meadows in combination with halophytic communities on solontschak and halophytic steppe communities; U14 - Pontic hardwood alluvial forests in combination with poplar and willow alluvial forests, partly with alder carrs.

Рис. 1. А) Розташування курганів в Полтавській, Черкаській та Кіровоградській областях; В) Розміщення території дослідження відносно різних типів степів в Україні (Map of the natural vegetation of Europe, Bohn et al. 2000): D57 - Південноєвропейсыкі ксерофітні різнотравні і багато злакові соснові та дубово-соснові ліси на неглибоких карбонатних грунтах зі степовими рослинами; F41 - Українські Східно-Подільські вапнові черешчатодубово - грабові ліси; F44 - Подільсько-Молдавські термофільні грабовочерешчатодубові ліси; L3 - Молдавсько-Українські лучні степи, що чергуються 3 грабово черешчатодубовими лісами, а в південній частині 3 татарськокленово - черещатодубовими лісами; L4 Південносарматські лучні степи, що чергуються 3 галофітною рослинністю на засолених грутнах та черещатодубовими лісами; М1 - Західно- та Центральнопонтичні багаторізнотравно злакові степи; Р34 Західно- та Центральнопонтичні залолені луки, що комбінуються 3 галофітними угрупованнями на солончаках і галофітизованими степовими угрупованнями; U14 - Понтичні алювіальні листяні ліси, що комбінуються 3 тополево-вербовими алювіальними лісами, 3 домішкою вільхових.

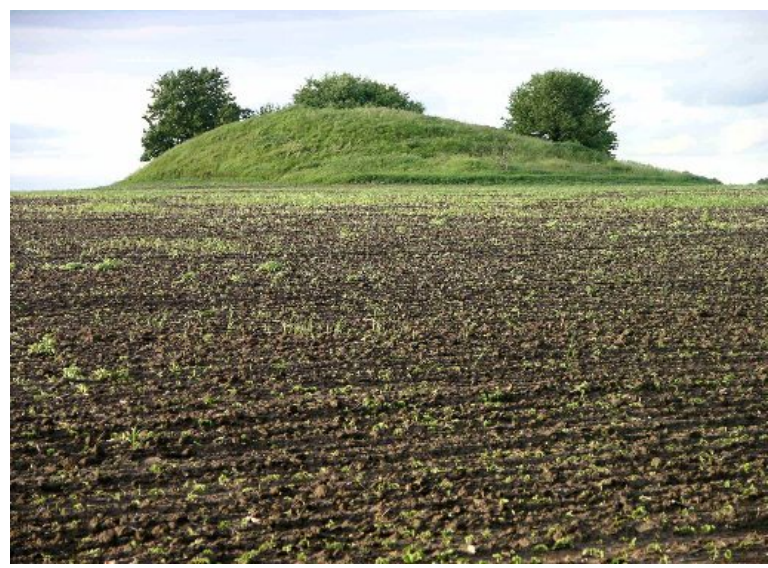

A)

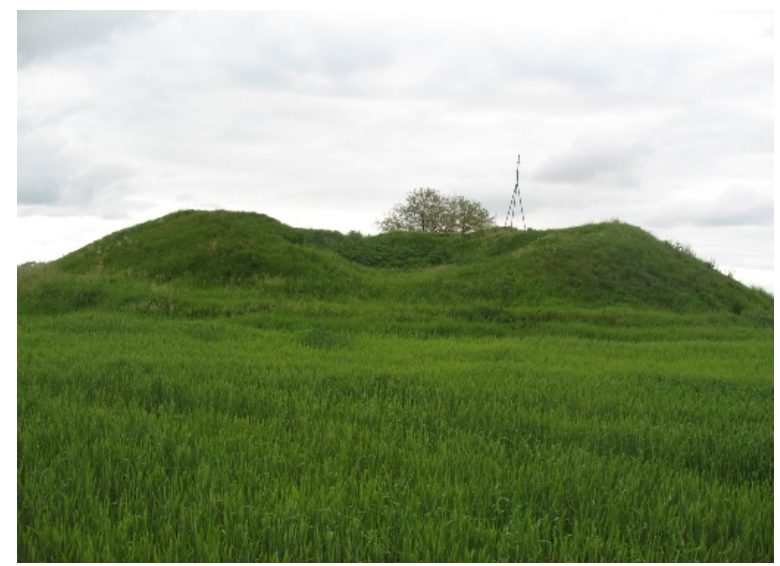

B)

Fig. 8. Different kinds of kurgans: A) typical kurgan, B) "maidan".

Рис. 8. Різні виду курганів: А) типовий курган, В) “майдан”. 
Location and size of the investigated kurgans in the forest steppe zone in Poltava, Cherkasy and Kirovograd Regions

Табиця 1

Локалізація та розміри досліджених курганів в лісостеповій зоні на території Полтавської, Черкаської та Кіровоградської областей

\begin{tabular}{|c|c|c|c|c|c|}
\hline $\begin{array}{l}\text { Code of the } \\
\text { kurgan }\end{array}$ & $\begin{array}{c}\text { Location } \\
\text { (nearest village) }\end{array}$ & $\begin{array}{l}\text { Longitude } \\
\text { (E) }\end{array}$ & $\begin{array}{l}\text { Latitude } \\
\text { (N) }\end{array}$ & $\begin{array}{c}\text { Height of } \\
\text { kurgan }(\mathrm{m})\end{array}$ & $\begin{array}{c}\text { Diameter } \\
\text { of kurgan } \\
(\mathrm{m})\end{array}$ \\
\hline \multicolumn{6}{|c|}{ Kirovograd Region } \\
\hline \multicolumn{6}{|c|}{ Aleksandriya District } \\
\hline F1 & Dibrova & $33^{0} 03^{\prime} 02.3^{\prime \prime}$ & $48^{0} 47^{\prime} 17.7^{\prime \prime}$ & 6 & 70 \\
\hline F2 & Nova Osota & $32^{0} 17^{\prime} 10.6^{\prime \prime}$ & $48^{0} 59^{\prime} 21.4^{\prime \prime}$ & 4 & 70 \\
\hline \multicolumn{6}{|c|}{ Poltava Region } \\
\hline \multicolumn{6}{|c|}{ Kremenchuk District } \\
\hline F3 & Pidgirne - Pavlysh & $33^{0} 22^{\prime} 32.7^{\prime \prime}$ & $48^{0} 57^{\prime} 18.1^{\prime \prime}$ & 7 & 60 \\
\hline F4 & Pidgirne - Pavlysh & $33^{0} 23^{\prime} 21.7^{\prime \prime}$ & $48^{0} 57^{\prime} 04.0^{\prime \prime}$ & 3 & 45 \\
\hline F5 & Bilets`kivka - Burty & $33^{0} 20^{\prime} 34.3^{\prime \prime}$ & $48^{0} 58^{\prime} 43.8^{\prime \prime}$ & 3 & 40 \\
\hline F6 & Yalyntsi, „3 Braty“ & $33^{0} 19^{\prime} 43.3^{\prime \prime}$ & $49^{0} 10^{\prime} 37.7^{\prime \prime}$ & 4 & 55 \\
\hline F7 & Yalyntsi, ,3 Braty“ & $33^{0} 19^{\prime} 47.4^{\prime \prime}$ & $49^{0} 10^{\prime} 36.5^{\prime \prime}$ & 3,5 & 50 \\
\hline \multicolumn{6}{|c|}{ Kirovograd Region } \\
\hline \multicolumn{6}{|c|}{ Onufriivka District } \\
\hline F8 & Onufriivka - Pavlysh & $33^{0} 25^{\prime} 19.6^{\prime \prime}$ & $48^{0} 54^{\prime} 51.3^{\prime \prime}$ & 6 & 60 \\
\hline F9 & Pavlysh - Kamburliivka & $33^{0} 23^{\prime} 00.0^{\prime \prime}$ & $48^{0} 53^{\prime} 38.4^{\prime \prime}$ & 4,5 & 50 \\
\hline F10 & Pavlysh - Kamburliivka & $33^{0} 22^{\prime} 25.2^{\prime \prime}$ & $48^{0} 53^{\prime} 29.8^{\prime \prime}$ & 7,5 & 65 \\
\hline \multicolumn{6}{|c|}{ Poltava Region } \\
\hline \multicolumn{6}{|c|}{ Svitlovods'k District } \\
\hline F11 & Fedorky & $32^{0} 47^{\prime} 44.2^{\prime \prime}$ & $48^{0} 54^{\prime} 38.8^{\prime \prime}$ & 5 & 50 \\
\hline \multicolumn{6}{|c|}{ Poltava Region } \\
\hline \multicolumn{6}{|c|}{ Globyne District } \\
\hline F12 & Ustymivka & $33^{0} 13^{\prime} 38.7^{\prime \prime}$ & $49^{0} 19^{\prime} 36.6^{\prime \prime}$ & 6.5 & 60 \\
\hline \multicolumn{6}{|c|}{ Cherkasy Region } \\
\hline \multicolumn{6}{|c|}{ Kamyanka District } \\
\hline F13 & Kamyanka - Grushkivka & $32^{0} 08^{\prime} 30.7^{\prime \prime}$ & $49^{\circ} 03^{\prime} 48.5^{\prime \prime}$ & 4,5 & 80 \\
\hline \multicolumn{6}{|c|}{ Cherkasy Region } \\
\hline \multicolumn{6}{|c|}{ Chyhyryn District } \\
\hline F14 & Chygyryn & $32^{0} 39^{\prime} 44.7^{\prime \prime}$ & $49^{0} 03^{\prime} 33.9^{\prime \prime}$ & 4 & 55 \\
\hline F15 & Chygyryn & $32^{0} 39^{\prime} 48.6^{\prime \prime}$ & $49^{\circ} 03^{\prime} 47.3^{\prime \prime}$ & 4 & 40 \\
\hline F16 & Chygyryn - Subotiv & $32^{0} 35^{\prime} 32.8^{\prime \prime}$ & $49^{0} 03^{\prime} 50.3^{\prime \prime}$ & 3,5 & 38 \\
\hline F17 & Chygyryn - Subotiv & $32^{0} 38^{\prime} 44.9^{\prime \prime}$ & $49^{\circ} 03^{\prime} 22.2^{\prime \prime}$ & 4 & 45 \\
\hline F18 & Cherneche & $32^{0} 39^{\prime} 29.6^{\prime \prime}$ & $49^{0} 02^{\prime} 26.7^{\prime \prime}$ & 6 & 60 \\
\hline \multicolumn{6}{|c|}{ Cherkasy Region } \\
\hline \multicolumn{6}{|c|}{ Chornobai District } \\
\hline F19 & Prydniprovs`ke, „Ostryuka” & $32^{0} 22^{\prime} 16.8^{\prime \prime}$ & $49^{0} 28^{\prime} 34.5^{\prime \prime}$ & 7,5 & 65 \\
\hline F20 & Lyashchivka & $32^{0} 40^{\prime} 15.5^{\prime \prime}$ & $49^{0} 29^{\prime} 44.9^{\prime \prime}$ & 5 & 45 \\
\hline F21 & Lyashchivka & $32^{0} 41^{\prime} 54.2^{\prime \prime}$ & $49^{0} 32^{\prime} 02.4^{\prime \prime}$ & 3,5 & 40 \\
\hline F22 & Lyashchivka & $32^{0} 42^{\prime} 05.7^{\prime \prime}$ & $49^{0} 32^{\prime} 18.2^{\prime \prime}$ & 5 & 40 \\
\hline \multicolumn{6}{|c|}{ Cherkasy Region } \\
\hline \multicolumn{6}{|c|}{ Smila District } \\
\hline F23 & Kutsivka & $31^{\circ} 39^{\prime} 13.8^{\prime \prime}$ & $49^{\circ} 02^{\prime} 54.0^{\prime \prime}$ & 6 & 55 \\
\hline F24 & Kutsivka & $31^{0} 37^{\prime} 11.8^{\prime \prime}$ & $49^{0} 02^{\prime} 37.6^{\prime \prime}$ & 5,5 & 65 \\
\hline F25 & Kutsivka & $31^{0} 37^{\prime} 57.5^{\prime \prime}$ & $49^{\circ} 02^{\prime} 42.1^{\prime \prime}$ & 6 & 50 \\
\hline
\end{tabular}

\section{Material and methods}

In the present work the same methods as those used in an earlier study [MOYSIYENKO, SUDNIK-WÓJCIKOWSKA 2006, 2009, SUDNIK-WÓJCIKOWSKA, MOYSIYENKO 2006] were applied. The following criteria were used to select the 25 kurgans: 
- kurgans more than $3 \mathrm{~m}$ high were chosen;

- good state of preservation of kurgans;

- the state of preservation of the plant cover; it was assumed that the presence of typical steppe species, such tuft grasses as Festuca valesiaca, Koeleria cristata and Stipa capillata or Bothriochloa ischaemum, Cleistogenes bulgarica was indicative of a relatively good condition of plant cover.

The flora of 5 microhabitats within 25 kurgans was investigated. The data were compiled in Table A (Appendix 1) which contained the following additional information about each taxon: its occurrence and abundance (estimated according to a 3-point scale: 1 sporadic, 2 - infrequent, 3 - common) in particular microhabitats within the kurgans investigated, species life form, its status in the historical-geographical classification, and origin in the case of alien species. Floristic analysis was conducted and the specific characters of the kurgan flora within forest steppe zone was determined. A five-grade scale was used to assess the frequency category of the species (see Fig. 3). The flora of 5 microhabitats within kurgans will be analysed in greater detail in another publication.

The species nomenclature follows S.L. MOSYAKIN, M.M. FEDORONCHUK [1999], Latin names of syntaxa are given according to В.А. СоломАХА [1996], Б.М. МиРКИН, Л.Г. НАУмОВА [1998], and W. MATUSZKIEWICZ [2001].

\section{Biodiversity of the kurgan flora}

\section{Results}

A total of 460 species of vascular plants were reported from 25 kurgans in the forest steppe zone (Appendix 1). The one additional species at the bottom of Table A was found on the kurgan in this zone which was not investigated in the present study (coordinates of this kurgan: Cherkasy Region, Chygyryn District, v. Medvedivka (N 52 10 '48.01', E $\left.32^{0} 21^{\prime} 18.71^{\prime \prime}\right)$. The number of species on particular kurgans ranged from 85 to $159,107,5$ on average. The kurgan flora in the forest steppe was richer in species than the flora of the barrows in each of the earlier investigated steppe zones (the desert steppe zone - 305 species [MOYSIYENKO, SUDNIK-WÓJCIKOWSKA 2006], in the west Pontic grass steppe zone - 352 [SUDNIK-WÓJCIKOWSKA, MOYSIYENKO 2006], and in the Pontic herb(-rich) grass steppe zone - 439 species [MOYSIYENKO, SUDNIK-WÓJCIKOWSKA 2009]). The species belonged to 256 genera and 51 families. The following families were represented by the greatest number of taxa: Asteraceae, Poaceae, Fabaceae, Lamiaceae, Caryophyllaceae, Rosaceae, Scrophulariaceae, Brassicaceae, Ranunculaceae, Apiaceae, Boraginaceae, Polygonaceae, Chenopodiaceae, Euphorbiaceae, Cyperaceae, Violaceae (Fig. 2). Compared with the flora of kurgans located in the other zones the role of the Caryophyllaceae and Ranunculaceae families increased. Genera represented by the highest number of taxa were, as follows: Veronica (15), Euphorbia (8), Carex (7), Potentilla (7), Salvia (7), Vicia (7), Viola (7), Artemisia (6), Trifolium (6).

About 191 species $(41,0 \%$ of the total kurgan flora) with the first (I) frequency class (Fig. 3) were considered sporadic or accidental (on 1-2 kurgans only). The group of common species recorded on 20-25 of the studied kurgans (frequency class V) made up about 4,8\% of the flora: Elytrigia repens (25), Koeleria cristata (25), Poa angustifolia (25), Convolvulus arvensis (24), Conyza canadensis (24), Chenopodium album (24), Artemisia absinthium (23), Euphorbia virgata (23), Linaria vulgaris (23), Bromopsis inermis (22), Falcaria vulgaris (22), Festuca valesiaca (22), Melandrium album (22), Salvia nemorosa (22), Setaria glauca (22), Sisymbrium polymorphum (22), Lactuca serriola (21), Medicago falcata (21), Achillea setacea (20), Agrimonia eupatoria (20), Gypsophila paniculata (20), Verbascum phoeniceum (20). 


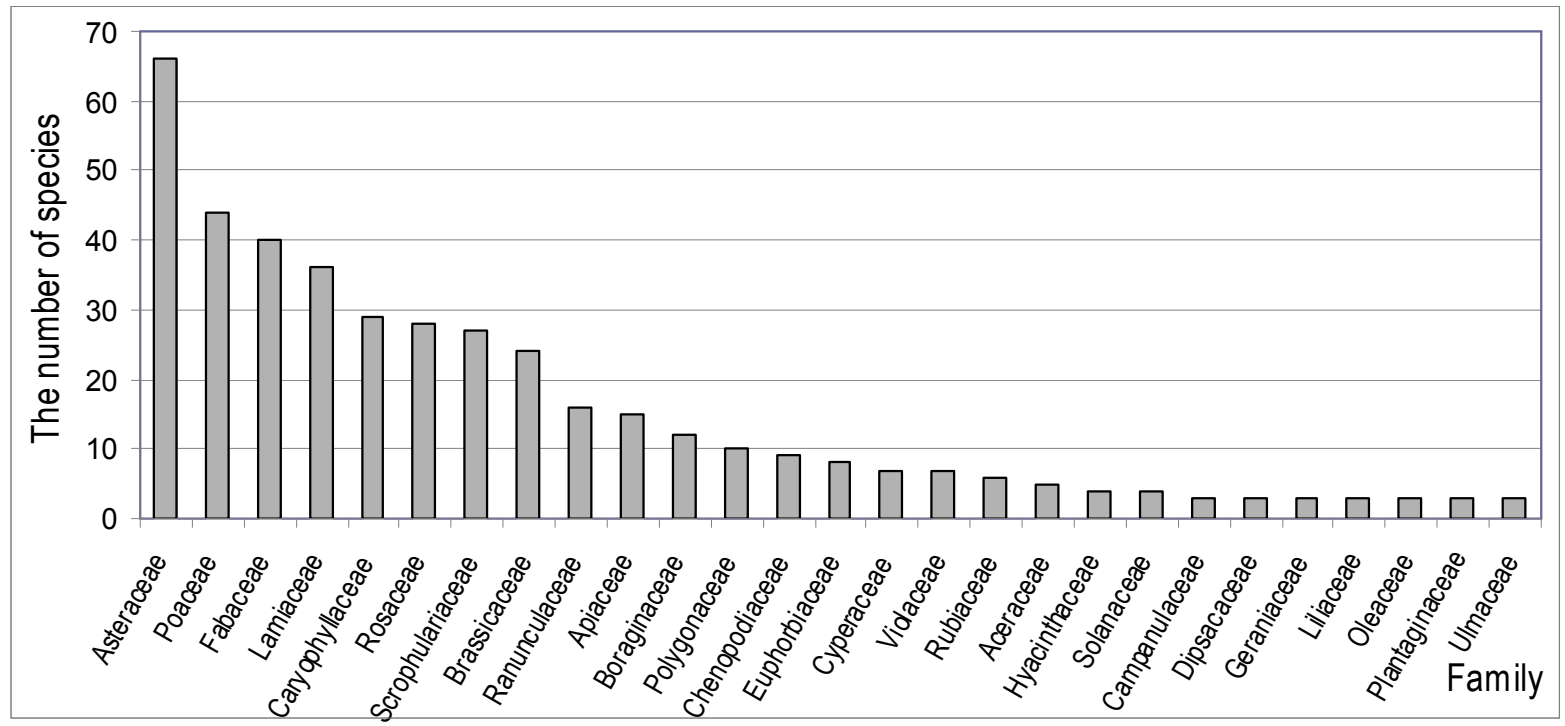

Fig. 2. The most important families (in terms of species number) in the total flora of kurgans in the forest steppe zone.

Рис. 2. Найбільш представлені (за кількістю видів) у флорі курганів лісостепової зони родини.

The total abundance of every species within the kurgans theoretically ranged from 0 up to 375 (a 3-grade scale was used to estimate the abundance of species, and 5 microhabitats within the 25 kurgans studied were taken into account: $3 \times 5 \times 25=375$ ). The abundance of only a few species exceeded 200, i.e.: Poa angustifolia (265) and Elytrigia repens (231). The abundance of 9 species ranged from 150 to 200: Convolvulus arvensis (198), Koeleria cristata (189), Festuca valesiaca (178), Euphorbia virgata (170), Salvia nemorosa (168), Medicago falcata (163), Sisymbrium polymorphum (156), Bromopsis inermis (151), Stipa capillata (150). At the same time, the above taxa were the most frequently encountered species.

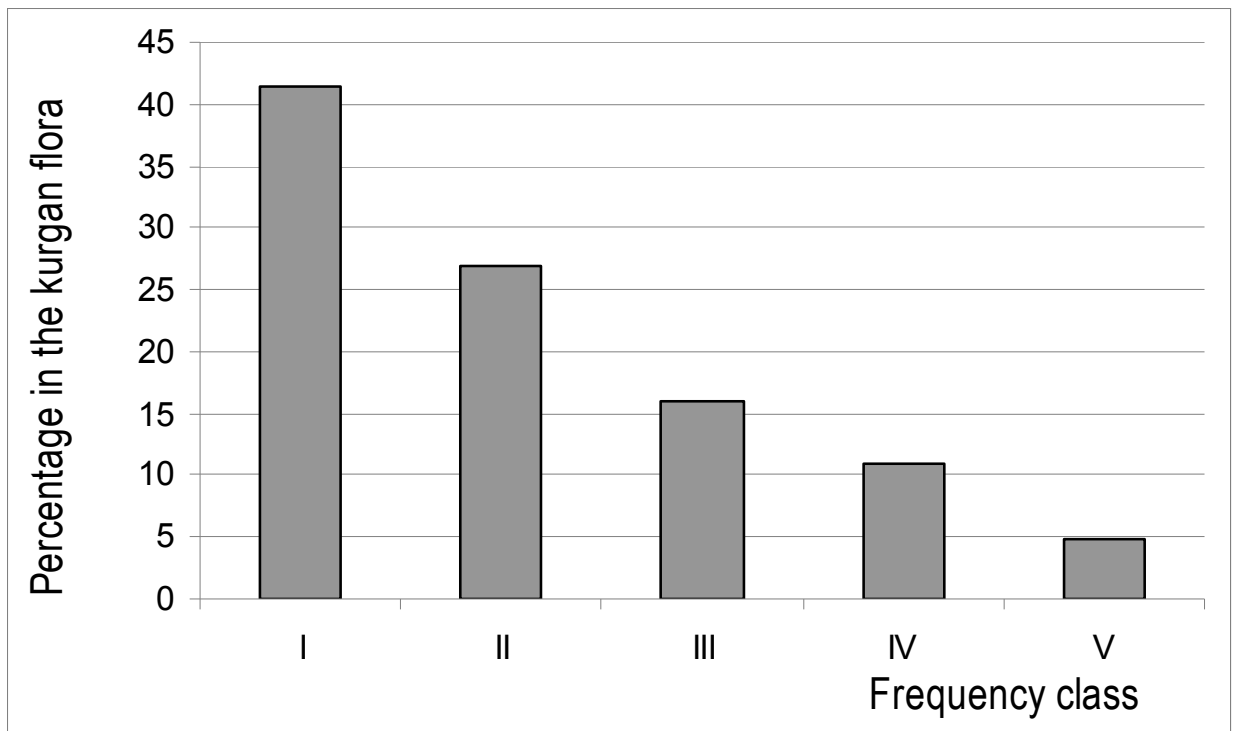

Fig. 3. Subdivision of the flora of kurgans in the forest steppe zone by class (the total number of species in each category is indicated at the top of the bar). Frequency classes: I - sporadic (1-2 kurgans), II - rare (36), III - relatively frequent (7-12), IV - frequent (13-19), common (20-25).

Рис. 3. Розподіл флори курганів лісостепової зони за класами частоти трапляння (на верхівці стовпчика вказана абсолютна кількість видів у кожній категорії). Класи частоти трапляння: I спорадично (1-2 кургани), II - рідко (3-6), III - досить часто (7-12), IV - часто (13-19), Звичайно (2025). 


\section{Spectrum of life forms}

The spectrum of life forms in the flora of kurgans in the forest steppe zone corresponded to that of the flora of the adjacent steppe zone. As well as in the Pontic herb(rich) grass steppe zone, hemicryptophytes were the dominating group of species $(39,4 \%)$. The second largest group were therophytes $(33,1 \%)$, which usually dominated in the west Pontic grass steppe zone (43,2\%), as well as in the desert steppe zone (46,5\%). Most of the therophytes ( 81 from 152), are alien species e.g. many weeds from the surrounding fields.

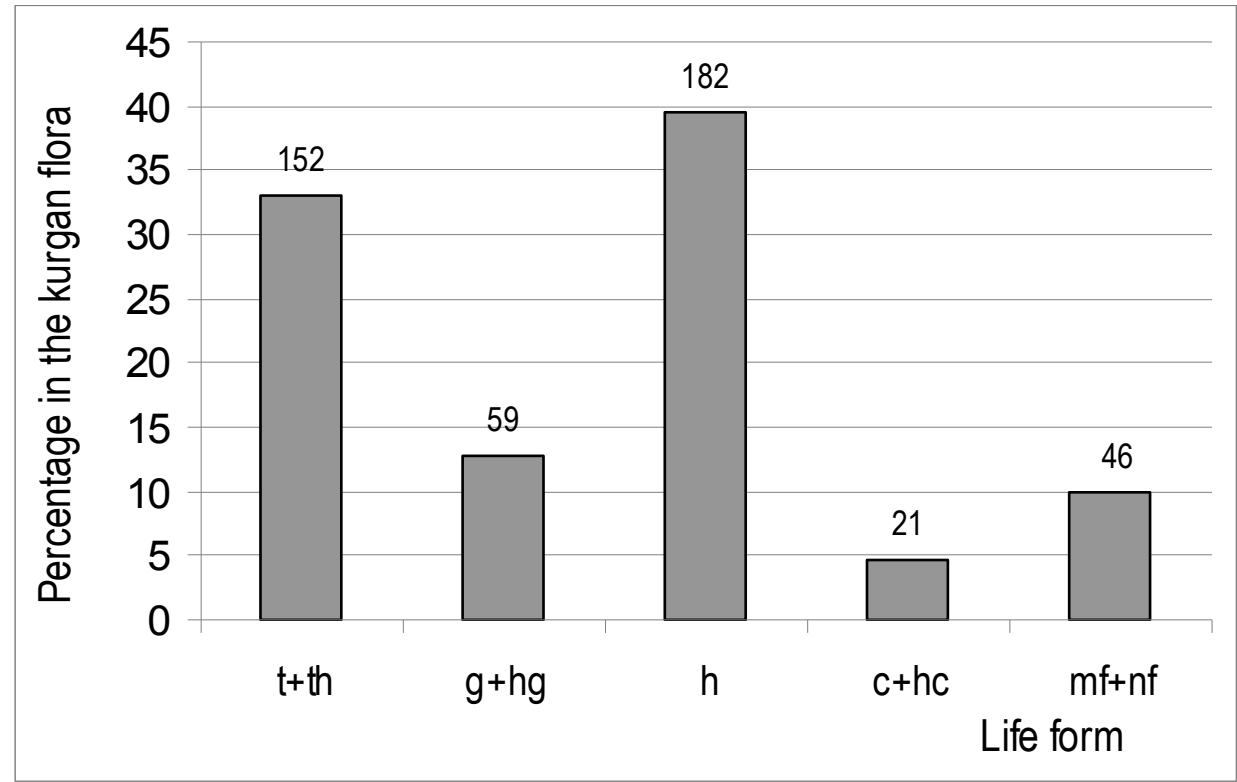

Fig. 4. Spectrum of life forms in the flora of kurgans in the forest steppe zone (for abbreviations see Appendix 1). The absolute number of species in each category is indicated at the top of the bar.

Рис. 4. Спектр життєвих форм флори курганів Лісостепу (прийняті скорочення дивись в Додатку 1). На верхівці стовпчика вказана абсолютна кількість видів у кожній категорії.

In the desert steppe zone phanerophytes comprised only $4 \%$ of the flora of kurgans, in the west Pontic grass steppe zone: 4,8\%. Their role in the flora of the barrows increased towards the north: $8,7 \%$ in the Pontic herb(-rich) grass steppe zone and 10,0\% in the forest steppe zone. The following species of native phanerophytes which were absent on the kurgans investigated in the zones further south were recorded: Chamaecytisus ruthenicus, Ch. lindemannii, Sambucus racemosa, Spiraea hypericifolia. In the case of the forest steppe zone "new" alien phanerophytes included: Amelanchier ovalis, Caragana arborescens, Cotinus coggygria, Ptelea trifoliata, Syringa vulgaris. Other life forms played a smaller role in the flora of the barrows: in the forest steppe zone geophytes comprised $12,9 \%$ of the kurgan flora, and chamaephytes $-4,6 \%$.

\section{Spectrum of socio-ecological groups}

The flora of kurgans was distinguished by a wide sociological range. It included the representatives of at least 20 syntaxa of higher ranks (Fig. 5). As in kurgans of the 3 steppe zones, species representing communities of the Festuco-Brometea and Stellarietea mediae classes had the biggest share concerning complex groups of steppe grasslands and synanthropic communities, $57,3 \%$, (i.e. $37,5 \%$, and $19,8 \%$ respectively). Such domination reflects the semi-natural and anthropogenic character of the kurgan flora.

Species associated with various steppe syntaxa: Festuco-Brometea, Festucetea vaginatae, Polygono-Artemisietea and Galietalia veri comprised as much as $49,3 \%$ of the kurgan flora $(37,5 \% ; 4,4 \% ; 1,5 \% ; 5,9 \%$ respectively). 


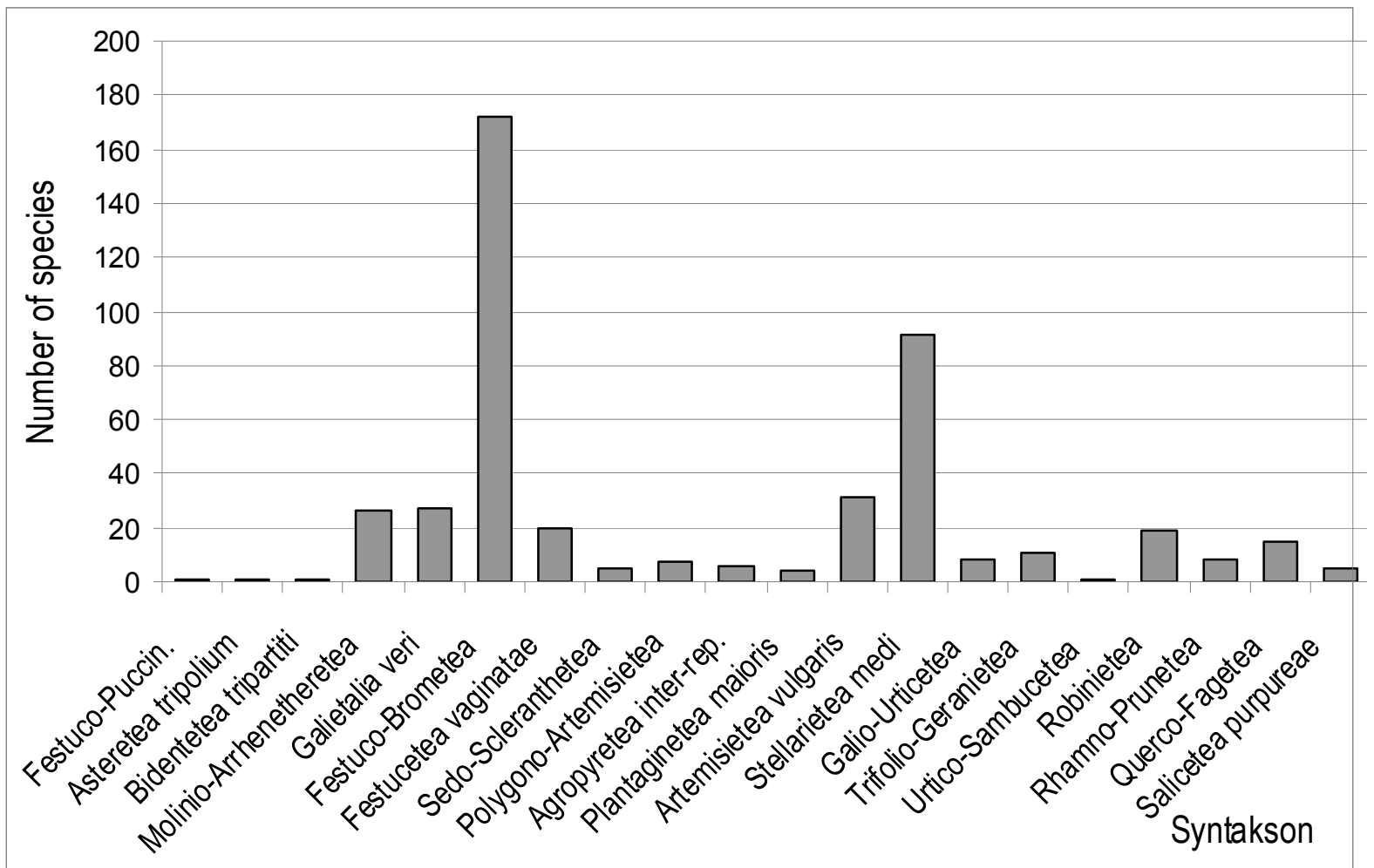

Fig. 5. The number of species from the syntaxa represented in the kurgan flora in the forest steppe zone (the order of syntaxa is not random - syntaxa given in a gradient from natural to synanthropic). For abbreviations see Appendix 1.

Рис. 5. Кількість видів в синтаксонах представлених на курганах в лісостеповій зоні (порядок синтаксонів не випадковий - в градієнті від натуральних до синантропних). Прийняті скорочення дивись в Додатку 1.

Species representing synanthropic syntaxa (with the exception of Stellarietea mediae), such as classes: Artemisietea vulgaris and Agropyretea intermedio-repentis as well as the Plantaginetalia majoris order $(6,8 \%, 1,3 \%, 0,9 \%$ respectively) constituted $28,8 \%$ of the kurgan flora.

Under more favourable soil moisture conditions, species belonging to the classes Molinio-Arrhenatheretea (except Galietalia veri) and Bidentetea were found growing at the foot of the kurgans, mostly on their northern side. However, their proportion in the flora of kurgans was estimated at $5,7 \%$ and $0,2 \%$ respectively.

Species associated with forest and scrub communities were a slightly better represented in the flora of kurgans in the forest steppe zone than in the Pontic herb(-rich) grass steppe zone and much better than in the grass steppe and desert steppe zones. A higher number of syntaxa which were represented by a big number of species were recorded. A total of 5 classes of tree and shrub communities were represented on the kurgans in the forest steppe zone: Querco-Fagetea, Urtico-Sambucetea, Robinietea and Salicetea purpureae. Species associated with the above syntaxa constituted 10,3\% of the flora of kurgans. The plant communites from the above classes were usually found at the foot of the kurgans. Other syntaxa of higher ranks, including halophyte communities (Asteretea tripolii, FestucoPuccinellietea) were poorly represented on the kurgans.

\section{Spectrum of species groups in the historical-geographical classification of plants}

The spectrum of synanthropic species groups in the flora of kurgans in the forest steppe zone (Tab. 2, Fig. 6) corresponded basically with the spectra for the earlier investigated 3 steppe zones. 


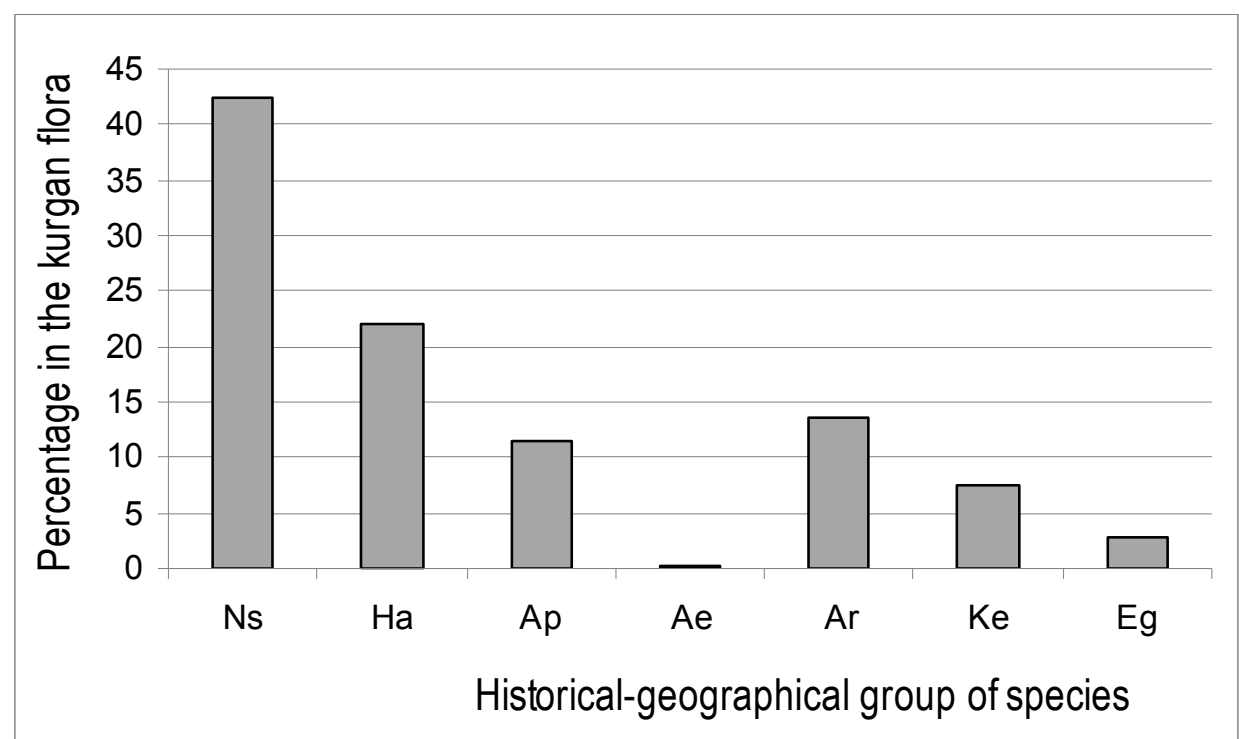

Fig. 6. Historical-geographical classification of the total flora of kurgans in the forest steppe zone. For abbreviations see Appendix 1 and Table 2.

Рис. 6. Географічно-історична класифікація флори курганів лісостепової зони. Прийняті скорочення дивись в Додатку 1 та Таблиці 2.

Native species accounted for $76,1 \%$ of the flora of kurgans in the investigated zone whereas non-synanthropic plants comprised $42,4 \%$ of the kurgan flora. The most frequently occurring species (frequency class V) were: Agrimonia eupatoria, Festuca valesiaca, Gypsophila paniculata, Koeleria cristata, Salvia nemorosa, Sisymbrium polymorphum, Verbascum phoeniceum. The percentage of native and non-synanthropic species in the flora of kurgans was similar as in the case of 2 steppe zones: in the Pontic herb(-rich) grass steppe zone $74,2 \%$ and $41,4 \%$, in the desert steppe zone: $77,2 \%$ and $40,9 \%$ respectively. Differences were bigger in the case of the west Pontic grass steppe zone: $70,8 \%$ and $39,5 \%$, respectively.

Depending on the level of transformation of the habitats into which they penetrate, apophytes can be subdivided into 3 groups: hemiapophytes $(22,0 \%$ of the total flora) and euapophytes + apophytes-oekiophytes $(11,7 \%)$ (Fig. 6). With respect to frequency, hemiapophytes distinctly predominated over euapophytes; 14 hemiapophytes belonged to frequency class V (Achillea setacea, Bromopsis inermis, Euphorbia virgata, Falcaria vulgaris, Medicago falcata, Poa angustifolia, but only 8 euapophytes represented the above frequency class (Chenopodium album, Convolvulus arvensis, Elytrigia repens, Linaria vulgaris, Melandrium album). The abundance of hemiapophytes is twice as high as that of euapophytes (3077 and 1391 respectively).

The total number of species of alien origin (anthropophytes) amounted to 110 ( 80 of them are therophytes). They comprised $23,9 \%$ of the flora of kurgans in the forest steppe zone and represented mainly families such as: Asteraceae (18 species), Brassicaceae (13), Poaceae (11), Fabaceae (7), Rosaceae (7) and Lamiaceae (7). Archaeophytes dominated among alien species $(13,7 \%$ of the total flora of kurgans; the abundance of 63 species of archaeophytes was estimated at 1222). The most frequently occurring archaeophytes (frequency class V) were: Artemisia absinthium, Lactuca seriola, Setaria glauca; frequency class IV: Ballota nigra, Capsella bursa-pastoris, Carduus nutans, Cichorium intybus, Descurainia sophia, Fallopia convolvulus, Lappula squarrosa, Lathyrus tuberosus, Setaria viridis, Sisymbrium loeselii, Viola arvensis. Kenophytes were less numerous (34 species; 7,4\%) and their total abundance was estimated to be 485 . Only 1 species of kenophytes represented frequency class V (Conyza canadensis). 
The number and abundance of species in groups of historical-geographical classification of the flora of kurgans in the forest steppe zone

Таблиця 2

Кількість і рясність видів в групах географічно-історичної класифікації флори курганів лісостепової зони

\begin{tabular}{|c|c|c|c|c|}
\hline & \multicolumn{2}{|c|}{$\begin{array}{l}\text { Species in historical- } \\
\text { geographical group }\end{array}$} & \multicolumn{2}{|c|}{$\begin{array}{l}\text { Total abundance of } \\
\text { species in historical- } \\
\text { geographical group }\end{array}$} \\
\hline & Number & $\%$ & Number & $\%$ \\
\hline Indigenous species: & 350 & 76,1 & 8714 & 83,3 \\
\hline Non-synanthropes (Ns) & 195 & 42,4 & 4219 & 40,3 \\
\hline Apophytes: & 155 & 33,7 & 4495 & 43,0 \\
\hline - Hemiapophytes (Ha) & 101 & 22,0 & 3077 & 29,4 \\
\hline - Euapophytes (Ap) & 53 & 11,5 & 1391 & 13,3 \\
\hline - Oekiophytes (Ae) & 1 & 0,2 & 27 & 0,3 \\
\hline Anthropophytes: & 110 & 23,9 & 1750 & 16,7 \\
\hline Archaeophytes (Ar) & 63 & 13,7 & 1222 & 11,7 \\
\hline Kenophytes (Ke) & 34 & 7,4 & 485 & 4,6 \\
\hline Ergasiophygophytes (Eg) & 13 & 2,8 & 43 & 0,4 \\
\hline Total flora & 460 & 100 & 10464 & 100 \\
\hline
\end{tabular}

The following species were included in frequency class IV: Amaranthus retroflexus, Armeniaca vulgaris. Ergasiophygophytes are a group of species which escaped from the cultivated fields surrounding the kurgans and became temporarily established on the barrows. They, however, made up only $2,8 \%$ of the total flora of kurgans.

The synanthropization index of the kurgan flora estimated from the proportion of apophyte and anthropophyte species in the flora of kurgans was more or less the same in the three steppe zones studied: $57,6 \%$ (in the desert steppe zone: $59,1 \%$, in the grass steppe zone: 60,5\%, and in the Pontic herb(-rich) grass steppe zone: 59,0\%). It is interesting to note that the percentage of the particular groups of synanthropic species was comparable in the three steppe zones.

However, some differences appeared when the geographical origin of the species was analyzed. It should be noted that the number of anthropophytes of various origins changed with the south-north direction: the share of Mediterranean-European species increased; in the desert steppe zone the contribution of Mediterranean-(Eur)asian species was much higher than that of Asian species. In the case of the grass steppe the differences in the proportion of the above two species groups were less pronounced, whereas in the Pontic herb(-rich) grass steppe as well as forest steppe zone Asian species played a more important role.

\section{Floristic values of the kurgans and the protection of the plant cover}

Similar problems exist concerning the conservation of the flora of kurgans in the case of the forest steppe zone and the steppe zones. The process of overgrowing of kurgans with trees and shrubs is much more strongly marked in the forest steppe than in the steppe zones. Shrubs and groups of trees occur more frequently and abundantly in this zone. They displace light-loving steppe species.

In the forest steppe zone we noted kurgans completely overgrown with forest, e.g. within a Scythian earthwork „Motroninskoye”, near Kholodnyi Yar and Melniki village (Chyhyryn District, Cherkasy Region). The steppe vegetation has been entirely destroyed on these kurgans. The forest has existed here for centuries. Partially overgrown kurgans are more often observed. Trees and shrubs are found growing at the foot and on the northern slopes of the kurgans. Steppe species are very rare in places densely overgrown with forests or shrubs 


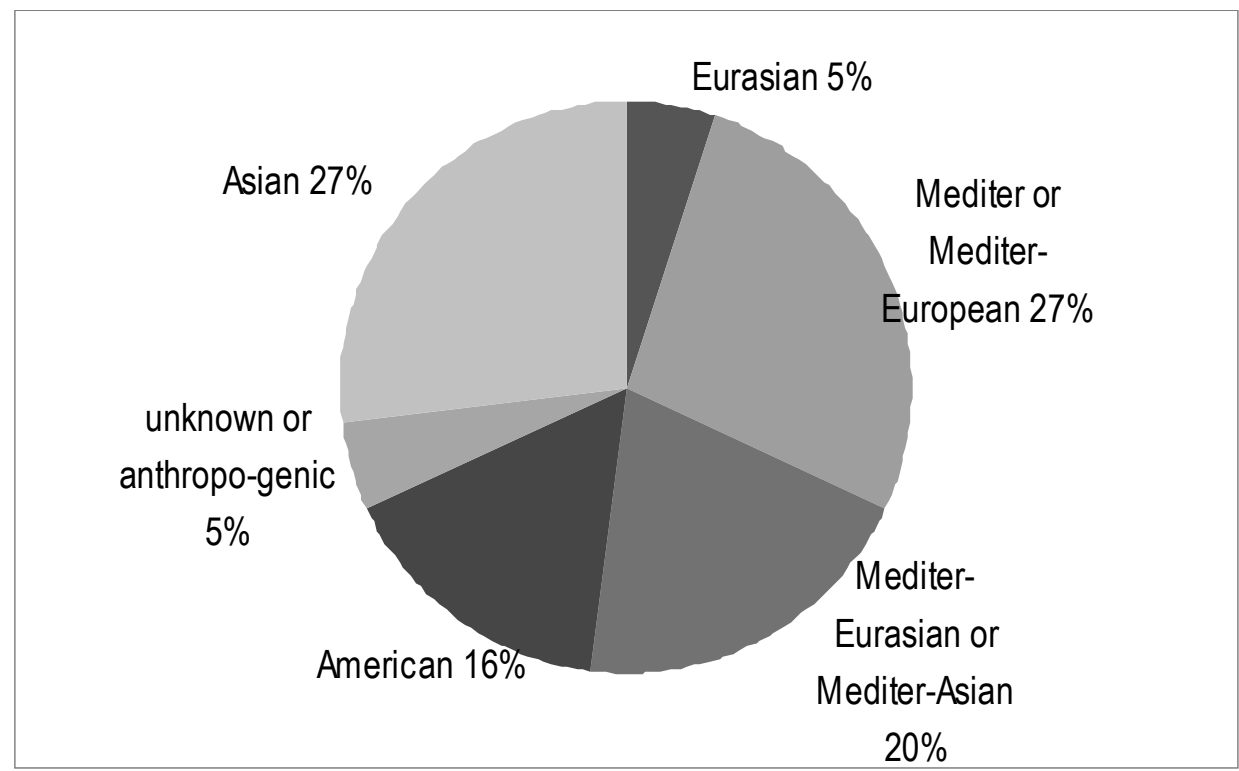

Fig. 7. Origin of the alien flora of kurgans in the forest steppe zone.

Рис. 7. Походження адвентивного елементу флори курганів лісостепової зони.

In the past the forest steppe zone was mainly inhabited by sedentary tribes (but penetrated by nomadic populations too) and the land was utilized agriculturally much earlier than the areas of southern Ukraine. These two factors had a significant effect on the flora of kurgans in the forest steppe. Special type of kurgans (ukr. "maidan") occur locally here (Fig. 8). We did not find any of these structures in the desert steppe zone and in the proper steppe zone during the course of our investigations. In the Middle Ages these kurgans were dug up and the soil enriched with saltpetre was gained from the central part of "maidans". Saltpetre was used by kozaks in production of gun-powder. Now, in these types of kurgans a characteristic deep "crater" may be observed. In the vicinity of these kurgans - piles of soil (ukr. "usy") - the remains of used soil from the inside of "maidans"is deposited. Changes in the shape of these kurgans also had an influence on the species composition of these structures.

The other problems concerning the anthropogenic transformation of the flora of kurgans were similar in the case of the forest steppe and the steppe zones. These problems were discussed in more detail in our earlier publications [МОЙСІєНКО, СУднІКВОЙцИКОВСЬКА, 2008, MOYSIYENKO, SUDNIK-WÓJCIKOWSKA, 2006, 2009; SUDNIKWóJCIKOWSKA, MOYSIYENKO, 2006]. The data presented below refer only to sozophytes recorded on the kurgans in the forest steppe.

Native species consist $76 \%$ of floristic list in the Appendix 1; among them 47 species are especially interesting (Fig. 9): Astragalus dasyanthus, Eremogone rigida, Linaria biebersteinii listed in "Plants of Ukraine in the 1997 IUCN - Red List of Threatened Plants" [Мосякін, 1999], also Astragalus dasyanthus from the "Europeaen Red List" [Червона книга..., 1996], Adonis vernalis, Pulsatilla pratensis, Stipa capillata, and also Astragalus dasyanthus - the "Red Data Book of Ukraine" [ЧЕРВОНА КНИГА..., 2009], Anthemis tinctoria (as Anthemis subtinctoria), Asyneuma canescens, Hyacinthella leucophaea, Iris pumila, Salvia austriaca, Salvia betonicaefolia, Vinca herbacea - the "Red Data List of Poltava Region" [БАЙРАК, СТЕЦЮК, 2005]", Amygdalus nana, Anemone sylvestris, Carex stenophylla, Cerasus fruticosa, Ephedra distachya, Goniolimon tataricum, Limonium tomentellum subsp. alutaceum (as L. alutaceum) Muscari neglectum, and also Hyacinthella leucophaea - the "Red Data List of Kirovograd Region" [ЗАПОВІДНІ.., 1999] and Allium flavescens, A. guttatum, Anchusa pseudoochroleuca, Aster bessarabicus, Astragalus varius, 
Carex supina, Cephalaria uralensis, Cleistogenes bulgarica, Elisanthe viscosa, Eremogone micradenia, Euphorbia kaleniczenkoi, Euphorbia subtilis, Galatella villosa, Helictotrichon pubescens, Hesperis tristis, Hieracium virosum, Iris hungarica, Jurinea calcarea, J. salicifolia, Lathyrus pannonicus, Linum hirsutum, Peucedanum alsaticum, Phlomis pungens, Salvia nutans, Seseli tortuosum, Thymus dimorphus, and also Amygdalus nana, Anemone sylvestris, Cerasus fruticosa, Iris pumila, Muscari neglectum, Salvia austriaca, Vinca herbacea - the "Red Data List of Cherkasy Region" [ШЕВчик та ін., 2006].

It appears that a considerable number of steppe species belongs to the above mentioned group of species of high conservation value [МОЙСІєНКО, СуднікВОЙЦИКОВСЬКА, 2008; MOYSIYENKO, SUDNIK-WÓJCIKOWSKA, 2006, 2009; SUDNIKWÓJCIKOWSKA, MOYSIYENKO, 2006]. This may be due to many reasons. The investigated kurgans in the forest steppe zone occurred over a much larger geographic area, i.e. within three regions, whereas the kurgans in the desert steppe zone within one region only, and in the grass steppe (Pontic herb-rich grass steppe and west Pontic grass steppe) within two regions. As a result, three local red lists of species were used in the case of the forest steppe, and 1-2 lists in the case of the steppe zone. In addition, a considerable number of steppe species, among others species typical of southern steppe areas, are included in the local floristic lists of species in the forest steppe zone, although the conditions prevailing within this zone are not optimal for this group of species. The flora of kurgans in the forest steppe is characterized by a higher proportion of sozophytes: from 2 to 21 species, 6,8 on average. The most valuable kurgans in terms of rare species were: F1 (13 species), F2 (12), F13 (21), F19 (15). Among the kurgans investigated in all the zones, kurgan F13 situated near Kamienka supported the highest number of rare species. The data pertaining to rare, valuable and protected species of plants on the kurgans in the forest steppe provide an additional argument that the kurgans in this zone should be under protection not only as archaeological sites but also as nature monuments (as in the case of the steppe zone).

Acknowledgements:

The study was supported by the Ministry of Science and Higher Education in Poland: grant NN 304081835 (2008-2011).

\section{References}

БАЙРАК О.М., СтЕцЮК Н.О. Атлас рідкісних і зникаючих рослин Полтавщини // Полтава: Верстка, 2005. $248 \mathrm{c}$.

ГЕОБОТАНІчнЕ РАЙОНУВАНня Української РСР / За ред. А.І. Барбарича // Київ: Наук. думка, 1977. - 305 с.

ДідУх Я.П., ШЕЛЯГ-СОСОНКО Ю.Р. Геоботанічне районування України та суміжних територій // Укр. Ботан. журн. - 2003. - Т. 60, № 1. - С. 6-17.

ЗАПОВІДНІ КУТОЧКИ КІРОВОГРАДСьКОї ЗЕМЛІ / Андрієнко Т.Л., Терещенко П.С., Клєстов М.Л. та ін. (під заг. ред. д.б.н. Т.Л. Андрієнко). - Київ: Арктур-А, 1999. - 240 с.

ЛАВРЕНКО Е.Н., КАРАМЫШЕВА З.В., НИКУЛИНА Р.И. Степи ЕвразИи. - Л.: Из-во «Наука», 1991. - 146 с.

МиРКИн Б.М., НАУмОВА Л.Г. Наука о растительности. - Уфа: Гилем, 1998. - 412 с.

МАРИнич О.М., ШищЕНКО П.Г. Фізична географія України. - Київ: Знання, 2003. - 479 с.

МосякІн С.Л. Рослини України у Світовому Червоному списку // Укр. ботан. журн. - 1999. - Т. 56, № 1. C. $79-88$.

Мойсієнко I.І., СуднІк-ВойциковсьКА Б. Созофіти у флорі курганів - рефугіумів степової флори на півдні України // Заповідна справа в Україні. - Т.14, Вип. 1. - 2008. - С.16-24.

ПриРодА Украинской ССР. Климат / Бабиченко В.Н., Барабаш М.Б., Логвинов К.Т. и др. (Отв. ред. Логинов К.Т., Щербань М.Г.) - К.: Наукова думка, 1984.-232 с.

ПриРОдА Украинской ССР. Почвы / Вернандер Н.Б., Гоголев И.Н., Ковалишин Д.И. и др. (Отв. ред. Н.Б.Вернандер, Д.А.Тютюнник). - К.: Наукова думка, 1986. - 214 с.

СоломаХА В.А. Синтаксономія рослинності України // Укр. фітоцен. зб. - 1996. - Сер. А, вип. 4 (5). $120 \mathrm{c}$.

ШЕВЧик В.Л., КУЗЕМКО А.А., ЧОРНА Г.А. Список рідкісних видів судинних рослин, що підлягають охороні в межах Черкаської області // Заповідна справа в Україні. - 2006. - Т. 12, № 1. - С. 11-17.

ЧЕРВОНА КНИГА України / Ю.Р.Шеляг-Сосонко (відп. ред.) та ін. - Київ: Вид-во Укр. енц., 1996. - 608 с.

ЧЕРвОНА КнигА України / Я.П.Дідух (ред.) та ін. - Київ: Вид-во «Глобалконсалтинг», 2009. - 912 с. 
MAP of the Natural vegetation of Europe, 1: 2500000 [KARTE der natürlichen Vegetation Europas, 1:2 500 000] / Bohn U., Gollub G., Hettwer C. et al. - Bonn: Bundesamt für Natürschutz, 2000. - Maps: 9 sheets. - Legend: 153 p.

MatuszKIEwiCz W. Przewodnik do oznaczania zbiorowisk roślinnych Polski. - Warszawa: Wydawnictwo Naukowe PWN, 2001. - 537 p.

Mosyakin S. L., FedoronchuK M. M. Vascular plants of Ukraine. A nomenclatural checklist. - Kiev: M. G. Kholodny Institute of Botany, National Academy of Sciences of Ukraine, 1999. - 346 pp.

MoYsiYenKo I.I., SuDNIK-WóJCIKOWSKA B., 2009: The flora of kurgans in the Pontic herb(-rich) grass steppe zone in Ukraine // Chornomors'k. bot. z. - Vol. 5, N 3. - P. 333-369.

MoysiYenko I.I., SUDNIK-WóJCIKOWSKA B. The Flora of Kurgans in the Desert Steppe Zone of Southern Ukraine // Chornomors'k. bot. z. - 2006. Vol. 2, N 1. - P. 5-35.

SudniK-WójCikowska B., Moysiyenko I.I. The flora of kurgans in the west Pontic grass steppe zone of southern Ukraine // Chornomors'k. bot. z. - 2006. Vol. 2, N 2. - P. 14-44.

Рекомендує до друку

Отримано 15.10 .2010 p.

Ан.В. Єна

Aдpecu aвmopis:

Barbara Sudnik-Wójcikowska,

Zakład Ekologii Roślin i Ochrony Środowiska,

Uniwersytet Warszawski,

Al. Ujazdowskie 4,

00-478 Warsaw, Poland;

e-mail:barbara.sudnik@uw.edu.pl

I.I. Мойсієнко

Кафедра ботаніки,

Херсонського державного університету,

Вул. 40 років Жовтня, 27, 73000 Херсон,

Україна, e-mail: Vanvan@ksu.ks.ua
Author's address:

Barbara Sudnik-Wójcikowska,

Department of Plant Ecology and Environmental Conservation,

University of Warsaw, Al. Ujazdowskie 4,

00-478 Warsaw, Poland;

e-mail: barbara.sudnik@uw.edu.pl

Ivan Moysiyenko,

Department of Botany,

Kherson State University,

Str. 40 let Oktriabrya 27, 73000 Kherson,

Ukraine; e-mail:Vanvan@ksu.ks.ua 


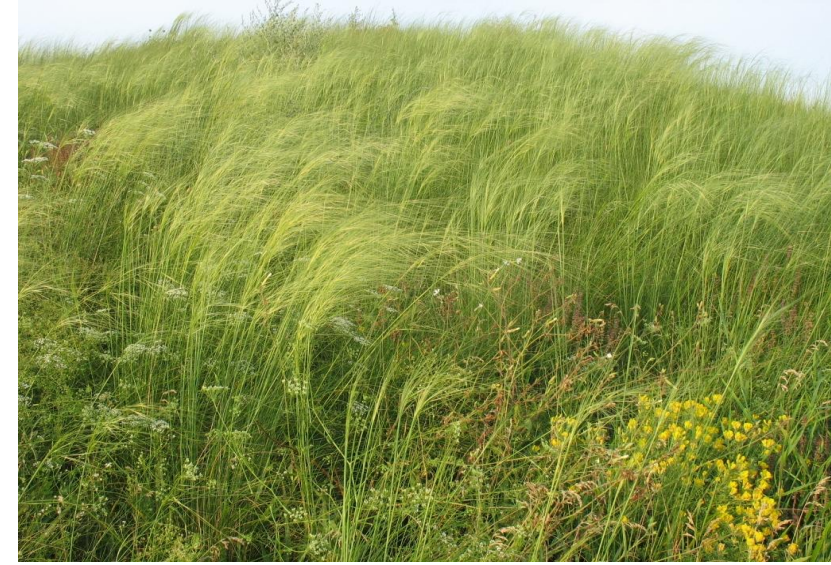

A)

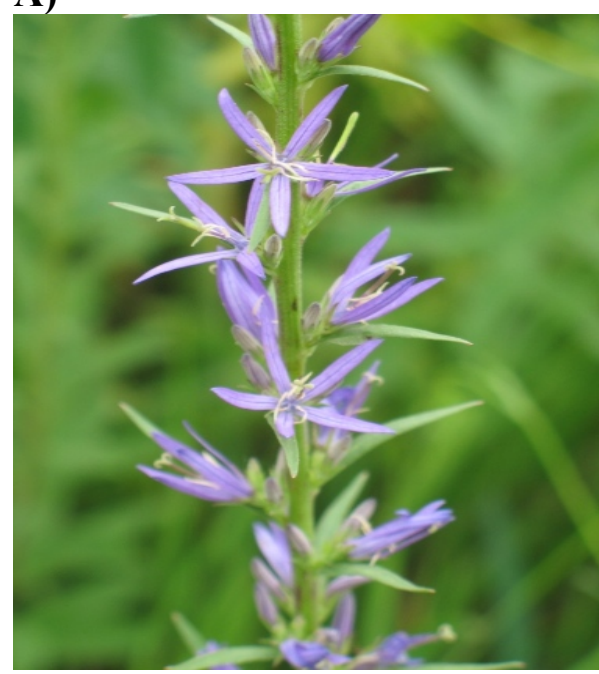

C)

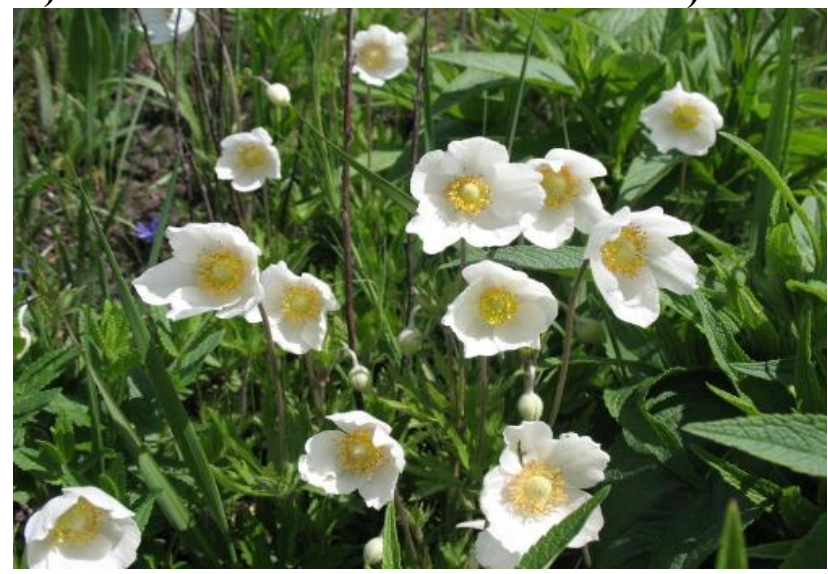

F)

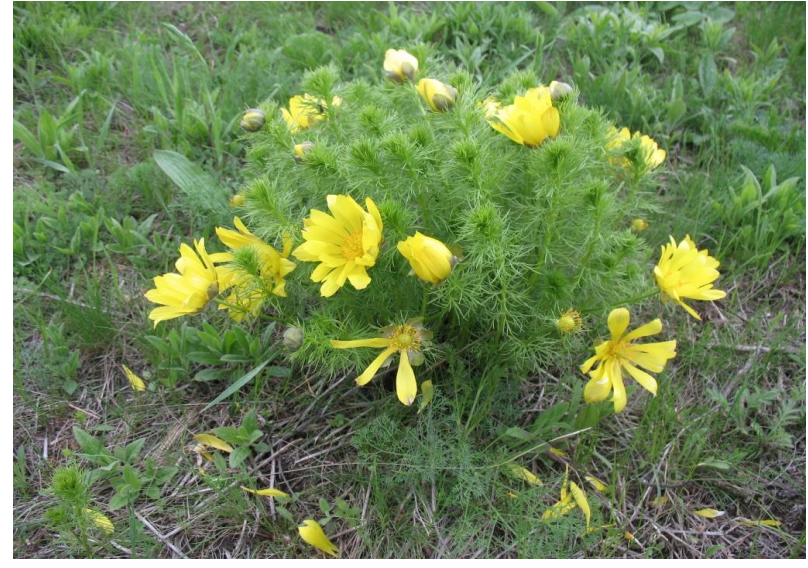

B)
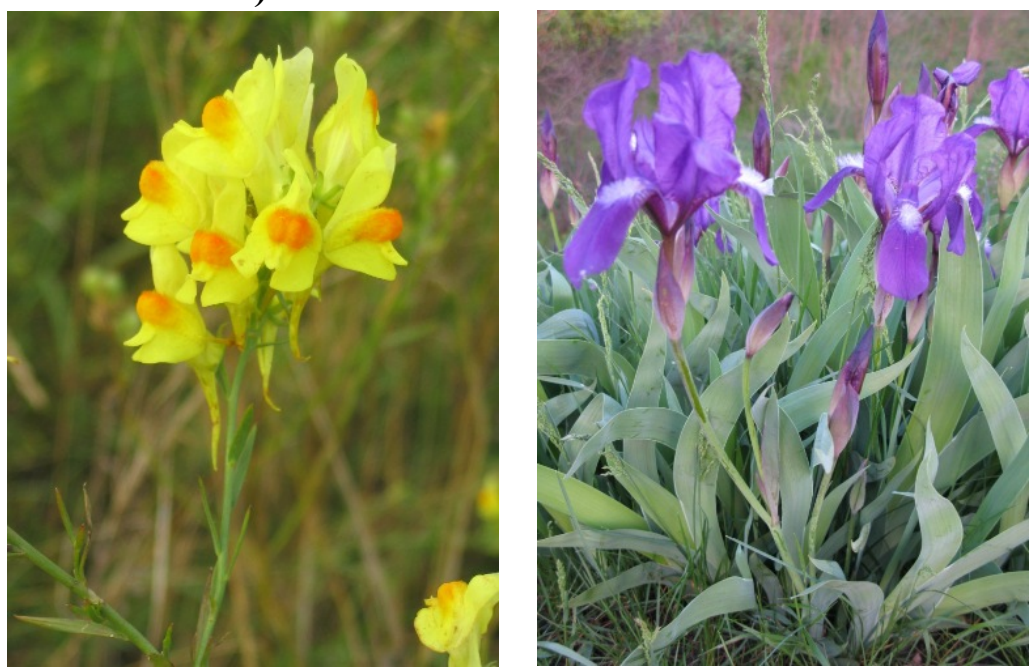

E)

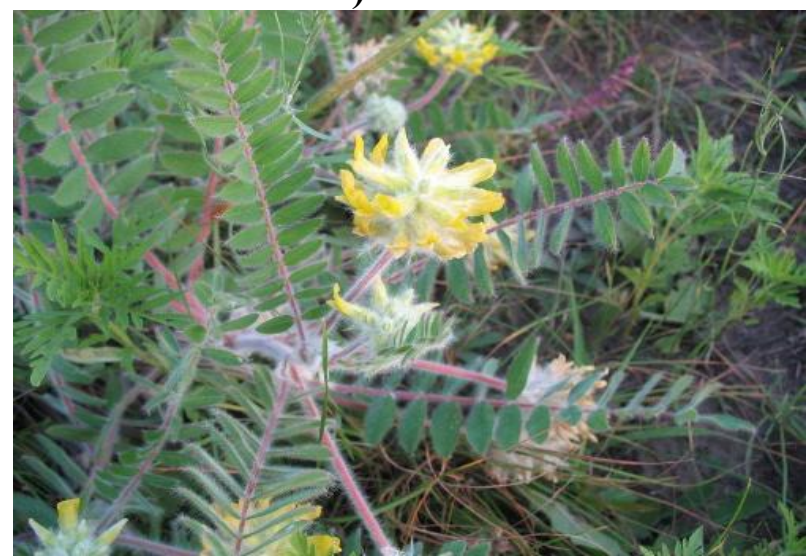

G)

Fig. 9. Sozophytes on the kurgans in the forest steppe zone: A) Stipa capillata, B) Adonis vernalis, C) Asyneuma canescens, D) Linaria biebersteinii, E) Iris hungarica, F) Anemone sylvestris, G) Astragalus dasyanthus.

Рис. 9. Созофіти курганів лісостепової зони: А) Stipa capillata, В) Adonis vernalis, C) Asyneuma canescens, D) Linaria biebersteinii, E) Iris hungarica, F) Anemone sylvestris, G) Astragalus dasyanthus. 
APPENDIX 1. Flora of the kurgans in desert steppe zone and their microhabitats

Abbreviations used in the Table A:

$\mathrm{T}$ - the top of the barrow;

\section{Microhabitats:}

Ss - the southern slopes;

$\mathrm{Sn}$ - the northern slopes;

Bs - the southern foot;

$\mathrm{Bn}$ - the northern foot.

Data regarding the occurrence of species in particular microhabitats are presented in the following order:

\begin{tabular}{|l|l|}
\hline \multicolumn{2}{|c|}{ T } \\
Ss & Sn \\
Bs & Bn \\
\hline
\end{tabular}

Life forms:

$\mathrm{t}$ - therophytes;

th - short-living perennials $(2,3,4$ years old);

g-geophytes;

w - hydrophytes;

h - hemicryptophytes;

hg - geophytes-hemicryptophytes - perennials, some of whose perennating buds (shoot system) remain on the soil surface and underground;

hc - hemicryptophytes-chamaephytes - perennials whose perennating buds remain on or above (within $0.25 \mathrm{~m}$ ) the soil surface;

c - chamaephytes;

$\mathrm{mf}$ - megaphanerophytes;

$\mathrm{nf}$ - nanophanerophytes;

Syntaxa:

Agro int-rep - Agropyretea intermedio-repentis (Oberd. et al. 1967) Mьller et Gцrs 1969) Alne glut-Alnetea glutinosae Br-Bl. et R. Tx. 1943

Alth offi-Althaetalia officinalis V. Golub et Mirkin in V. Golub 1995 \{Molinio-

Arrhenatheretea T. Tx 1937\}

Ammoph-Ammophiletea Br.-B1. et R.Tx. 1943

Artemi - Artemisietea vulgaris Lohm., Prsg et R. Tx. in R. Tx. 1950

Aste trip - Asteretea trifolium Westh. et Beeft. ap. Beeft. 1962

Bident-Bidentetea tripartiti R.Tx., Lohm. et Prsg. 1950

Caki mari-Cakiletea maritimae R. Tx. et Prsg. 1950

Crit-Limo - Crithmo-Limonietea Br.-B1. 1947

Crit-Stat-Crithmo-Staticetea Br.-B1. 1947

Fest vagi-Festucetea vaginatae Soy $1968 \mathrm{em}$. Vicherek 1972 or Festucetalia vaginatae

Soy $\{$ Festuco-Brometea Br.-Bl. et R. Tx. 1943\}

Fest-Brom - Festuco-Brometea Br.-Bl. et R. Tx. 1943

Fest-Pucc-Festuco-Puccinellietea Soy (incl. Festuco-Limonietea Karpov et Mirkin 1985)

Gali veri-Galietalia veri Mirkin et Naumova 1986 \{Molinio-Arrhenatheretea T. Tx. 1937\}

Gali-Urti-Galio-Urticetea Passarge 1967 or Galio-Urticenea (Passarge 1967)

\{Artemisietea Lohm., Prsg. et R. Tx. in R. Tx. 1950\}

Glecho-Glechometalia hederaceae R. Tx. in R. Tx.

Moli-Arrh-Molinio-Arrhenatheretea R. Tx. 1937

Phragmi - Phragmitetalia Koch 1926

Plan majo - Plantaginetea majoris T. Tx. et Prsg. 1950 or Plantaginetalia majoris R. Tx. (1943) 1950 \{Molinio-Arrhenatheretea R. Tx. 1937\} 
Poly-Arte - Polygono-Artemisietea austriacae Mirkin, Sakhapov et Solomeshch in Mirkin et al. 1986

Quer rob-pe-Quercetea robori-petraeae Br.-B1. et R. Tx. 1943

Quer pub-pe-Quercetalia pubescenti-petraeae Klika 1933 corr. Moravec in Beg. et

Theurill 1984

Quer-Fage - Querco-Fagetea Br.-Bl. et Vlieg. 1937

Rham-Prun - Rhamno-Prunetea Rivas, Goday et Garb. 1961\}

Robin - Robinietea Jurko ex Hadac et Sofron 1980

Sali purp - Salicetea purpureae Moor 1958

Sedo-Scle-Sedo-Scleranthetea Br.-B1. 1955

Stel medi - Stellarietea mediae T. Tx. , Lohm., et Prsg. 1950 (incl. Chenopodietea Br.-B1.

1952 em. Lohm., J. et R. Tx. 1961 ex Matuszk.1962 \& Secalietea Br.-Bl. 1951)

Trif-Gera - Trifolio-Geranietea sanguinnei Th. Mьller 1962

Urti-Samb - Urtico-Sambucetea Doing1962 em. Pass.1968

Vacc-Pice-Vaccinio-Piceetea Br-B1. 1939

[ ] - indicates that the introduced species were established in the particular plant community types (see Table);

\{\} - indicates that the syntaxon belongs to a given class (see above list of syntaxa abbreviations).

Historical-geographical classification of species:

Native species:

Ns - native species, not established in anthropogenic habitats;

Ap - true apophytes = euapophytes, natives established in anthropogenic habitats;

$\mathrm{Ha}$ - hemiapophytes, natives established only in semi-natural habitats;

$\mathrm{Ae}$ - oekiophytes, natives grown (e.g. in plantations or in windbreaks) and recorded in anthropogenic habitats.

Aliens:

$\mathrm{Ar}-$ archaeophytes, aliens that immigrated before the year 1500;

$\mathrm{Ke}$ - kenophytes, aliens introduced after the year 1500;

$\mathrm{Eg}$ - ergasiophygophytes, cultivated plants not established in the new territory, appearing only temporarily.

Origin of alien species - groups and abbreviations:

1 Mediterr (= Mediterranean), sub-Mediterr (= sub-Mediterranean);

2 European, Atlantic, sub-Atlantic;

3 Eurasian, Eurosiberian, boreal-Eurasian, continent. (= continental), subcontinent (= subcontinental);

4 W-Asian (=Western-Asian), Middle-Asian, C-Asian (= Central Asian), IranoTuranian, Indian, Malay;

5 African,

6 North American, Central American, South American.

Status of the protected species:

* - World Red List

** - European Red List

$* * *$ - Red Data Book of Ukraine

**** - Red Lists of Poltava Region, Kirovograd Region and Cherkasy Region.

At the bottom of the Table A the flora of each kurgan is described taking into account:

1 the number of species;

2 the number of species in each of its microhabitats. 


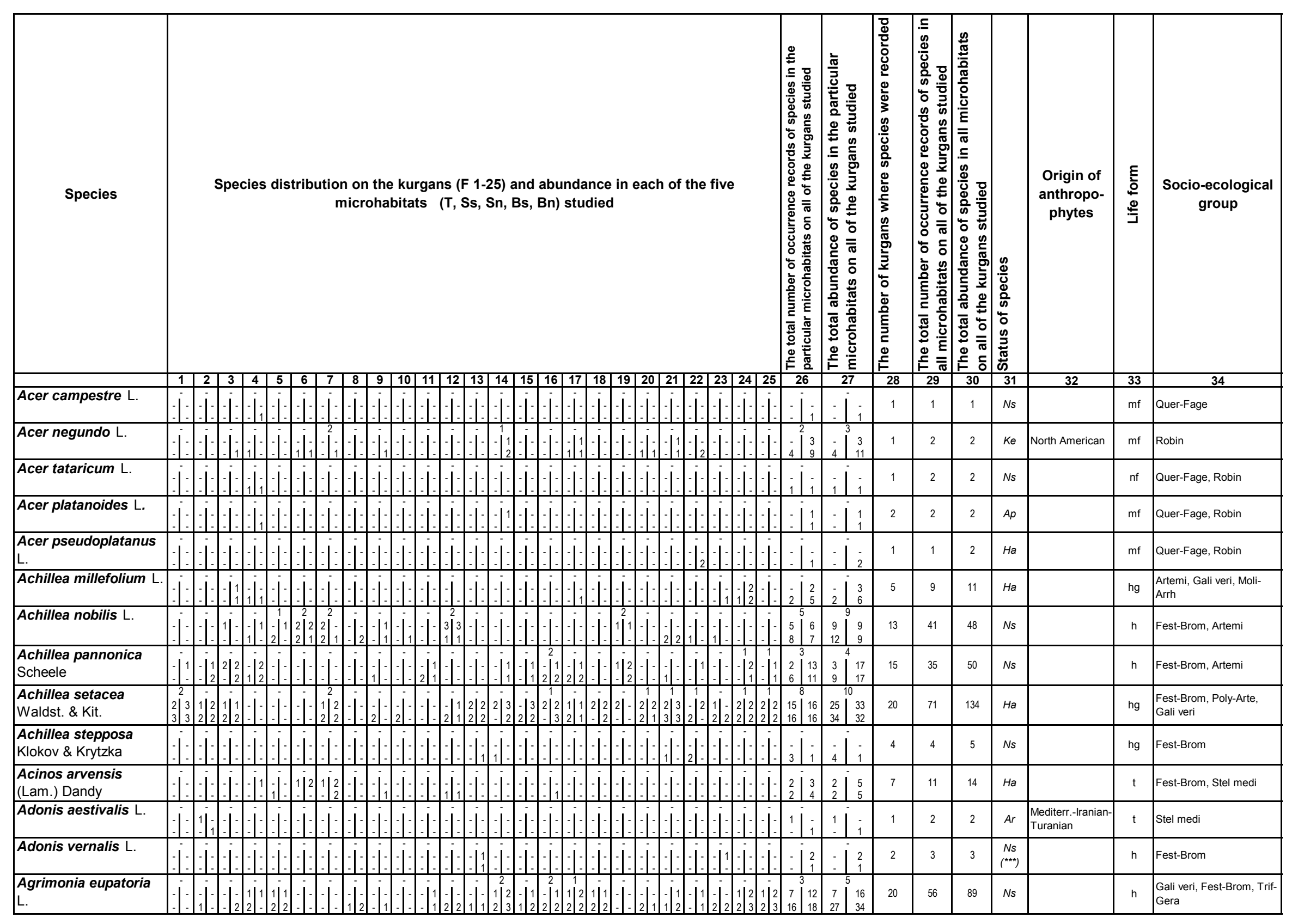




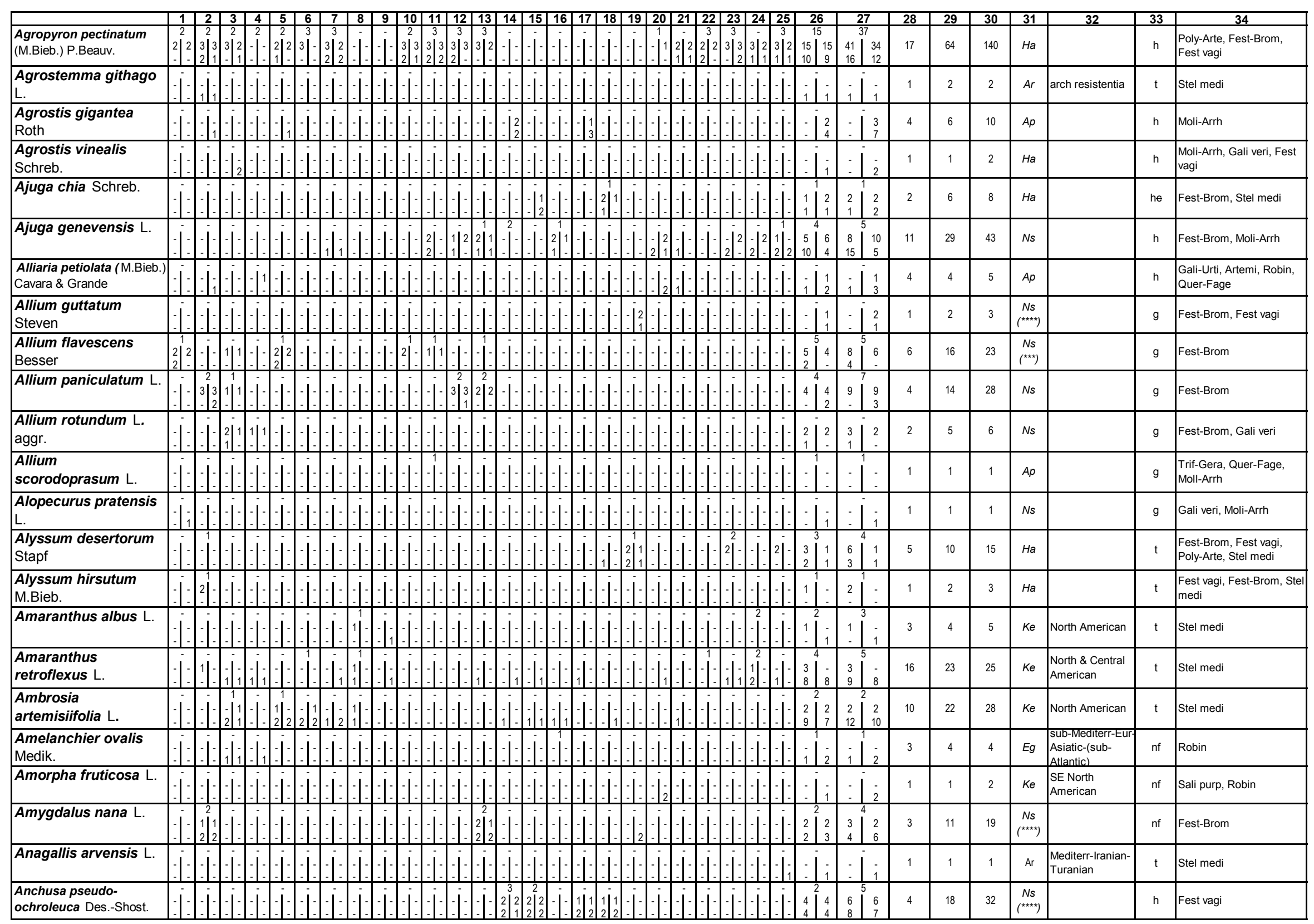




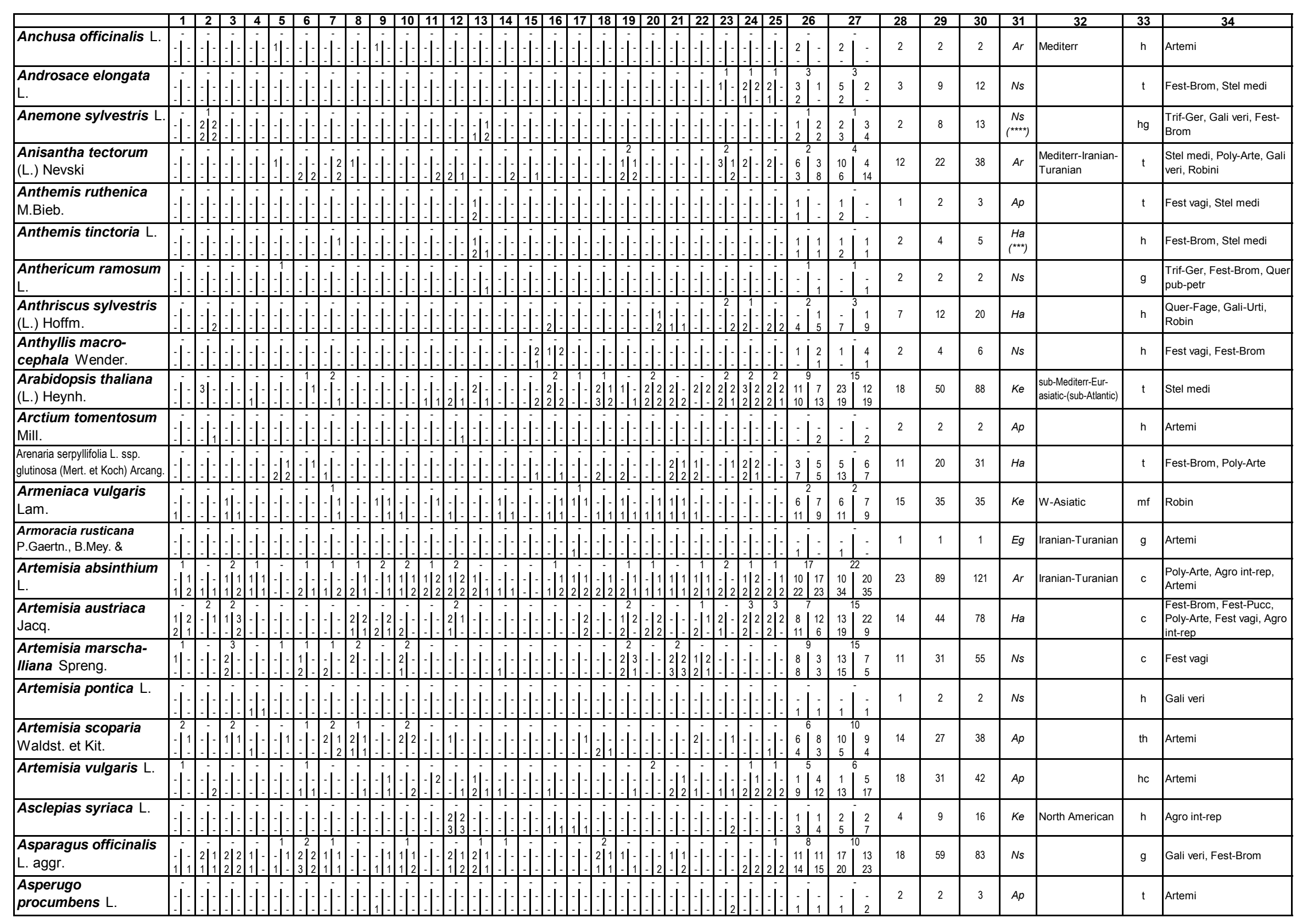




\begin{tabular}{|c|c|c|c|c|c|c|c|c|c|c|c|c|c|c|c|c|c|c|c|c|c|c|c|c|c|c|c|c|c|c|c|c|c|c|}
\hline & \begin{tabular}{|l|}
1 \\
-
\end{tabular} & & \begin{tabular}{|l|l}
3 & \\
\end{tabular} & 4 & \begin{tabular}{|l|}
5 \\
\end{tabular} & & \begin{tabular}{|l|}
7 \\
2 \\
\end{tabular} & 8 & & \begin{tabular}{|l|l|}
10 & 11 \\
\end{tabular} & & 13 & 14 & 15 & 16 & 17 & 18 & \begin{tabular}{|l|}
19 \\
\end{tabular} & 20 & 21 & \begin{tabular}{l|l}
22 & 2 \\
-
\end{tabular} & \begin{tabular}{|l|l|}
23 & 24 \\
- & -
\end{tabular} & 25 & & \begin{tabular}{l|l}
26 & \\
7 &
\end{tabular} & $\frac{\mathbf{2 7}}{12}$ & & 28 & 29 & 30 & 31 & 32 & 33 & 34 \\
\hline $\begin{array}{l}\text { Asperula cynanchica } \\
\text { L. }\end{array}$ & -2 & & & & \begin{tabular}{l|l}
2 & 2 \\
2 & 1 \\
\end{tabular} & & \begin{tabular}{|l|l|l|} 
& 2 \\
2 & 2 \\
& 1 & 1 \\
\end{tabular} & & & & & \begin{tabular}{ll|l|l|}
2 & 2 \\
2 & 1
\end{tabular} & & & & & $\left|\begin{array}{ll}2 \\
1 & 2 \\
1\end{array}\right|$ & & & & & -1 & 1 & & $\left|\begin{array}{l}16 \\
12\end{array}\right|$ & & $\begin{array}{l}2 \\
32 \\
18\end{array}$ & 19 & 62 & 110 & Ns & & $\mathrm{h}$ & Fest-Brom \\
\hline \begin{tabular}{|l} 
Aster bessarabicus \\
Bernh. ex Rchb.
\end{tabular} & -1 & & & & 1 & & & & & & & & & & & & & & & & & $2_{2}^{2}$ & $2=0$ & & \begin{tabular}{|l|}
2 \\
2 \\
\end{tabular} & & & 2 & 5 & 7 & \begin{tabular}{|c|}
$N s$ \\
$(* * * *)$ \\
\end{tabular} & & $\mathrm{h}$ & Fest-Brom, Gali veri \\
\hline $\begin{array}{l}\text { Astragalus asper } \\
\text { Jacq. }\end{array}$ & -1 & & & & & & & & & & & & & & & & & & & & & & -1 & & $\mid$ & $\begin{array}{l}1 \\
.\end{array}$ & & 1 & 1 & 1 & Ns & & $\mathrm{h}$ & Fest-Brom \\
\hline $\begin{array}{l}\begin{array}{l}\text { Astragalus austriacus } \\
\text { Jacq. }\end{array} \\
\end{array}$ & -1 & & & & 1 & & & & & -1. & & & & & & & & & & 1 & & -1 & 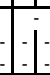 & & 2 & & - & 2 & 2 & 3 & Ns & & $\mathrm{h}$ & Fest-Brom \\
\hline $\begin{array}{l}\text { Astragalus } \\
\text { dasyanthus Pall. }\end{array}$ & \begin{tabular}{|l|l|} 
& 1 \\
1 & 1 \\
\end{tabular} & & & & \begin{tabular}{ll|}
2 & 3 \\
2 & 2 \\
\end{tabular} & & & & & & & & & & & & & & & & & & 1 & & \begin{tabular}{|l|}
2 \\
2 \\
\end{tabular} & & $\begin{array}{l}4 \\
3 \\
\end{array}$ & 2 & 8 & 13 & \begin{tabular}{|c|}
$N s$ \\
$(* * *)$ \\
\end{tabular} & & $\mathrm{h}$ & Fest-Brom \\
\hline \begin{tabular}{|l|} 
Astragalus \\
onobrychis L. \\
\end{tabular} & -1 & & & & -1 & & \begin{tabular}{|l|l|}
2 & 2 \\
1 & 2 \\
\end{tabular} & & & & & & & & & & & & & & & & & & \begin{tabular}{|l|}
6 \\
2 \\
\end{tabular} & $\begin{array}{l}8 \\
5 \\
\end{array}$ & $\begin{array}{l}8 \\
3 \\
\end{array}$ & 6 & 17 & 25 & Ns & & $\mathrm{h}$ & Fest-Brom \\
\hline $\begin{array}{l}\text { Astragalus varius } \\
\text { S.G.Gmel. }\end{array}$ & 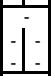 & & & & 1 & & & -1. & & -1 & & & & & & & & & & -1 & 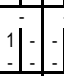 & 1 & -5 & & 11 & $\begin{array}{ll} & 1 \\
3 & 1 \\
3\end{array}$ & 2 & 2 & 5 & 9 & \begin{tabular}{|c|}
$N s$ \\
$(* * * *)$ \\
\end{tabular} & & $\mathrm{h}$ & Fest vagi, Fest-Brom \\
\hline $\begin{array}{l}\text { Asyneuma canescens } \\
\text { (Waldst. et Kit.)Griseb. et }\end{array}$ & -1 & & & & $1:$ & & $-1-1$ & & & $i$ & & & & & & & & & & & & -1 & $1-1$ & & & & & 3 & 3 & 3 & \begin{tabular}{|c|}
$N s$ \\
$(* * * *)$ \\
\end{tabular} & & $\mathrm{h}$ & Fest-Brom \\
\hline $\begin{array}{l}\text { Atriplex oblongifolia } \\
\text { Waldst. \& Kit. }\end{array}$ & $i$ & & & & & & & & & & & & & & & & & & & & & & $\begin{array}{ccc} & 1 \\
2 & -1 & -1 \\
\end{array}$ & & $\mid$\begin{tabular}{l|}
3 \\
3 \\
4
\end{tabular} & $\begin{array}{l}4 \\
4 \\
4\end{array}$ & $\begin{array}{l}5 \\
5 \\
5\end{array}$ & 8 & 16 & 23 & $\mathrm{Ha}$ & & $\mathrm{t}$ & Poly-Arte, Stel medi \\
\hline Atriplex patula $\mathrm{L}$. & 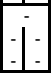 & & & & & & & & & $\therefore \frac{1}{2}$ & & & & & & & & & & & & $i$ & is & \begin{tabular}{|l}
1 \\
2 \\
2
\end{tabular} & $\left.\right|_{3} ^{2}$ & $a_{2}^{3}$ & 5 & 6 & 8 & 11 & $A p$ & & $\mathrm{t}$ & Stel medi, Robini \\
\hline $\begin{array}{l}\text { Atriplex sagittata } \\
\text { Borkh. }\end{array}$ & -1 & & & & 1 & & & & & 1 & & & & & & & & & & & & & . & 1 & $\perp$ & i & & 1 & 1 & 1 & Ar & Iranian-Turanian & $\mathrm{t}$ & Stel medi, Artemi \\
\hline Avena persica Steud. & $i$ & & & & & & & & & & & & & & & & & & & & & $-1-1$ & 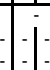 & & & & $\begin{array}{l}1 \\
2 \\
\end{array}$ & 1 & 3 & 4 & Ke & Mediterr & $\mathrm{t}$ & Stel medi \\
\hline Ballota nigra L. & & & & & & & & & & & & & & & & & & & & & & & 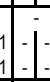 & & $\mid \begin{array}{c}8 \\
14 \\
\end{array}$ & \begin{tabular}{|l|}
13 \\
5 \\
18
\end{tabular} & $\begin{array}{l}30 \\
24 \\
\end{array}$ & 16 & 45 & 70 & Ar & Mediterr & hc & Artemi, Gali-Urti, Robin \\
\hline \begin{tabular}{|l|} 
Berteroa incana (L.) \\
DC. \\
\end{tabular} & \begin{tabular}{|l|l|} 
& 1 \\
& 1 \\
2 & 2 \\
\end{tabular} & & & & 1 & & \begin{tabular}{|r|r|r|r|}
- & 1 \\
2 & 1 \\
\end{tabular} & \begin{tabular}{|l|l|l}
2 & 1 \\
\end{tabular} & & $i$ & & & & & & & & & & & & 1 & ii & & $\left|\begin{array}{l}13 \\
12\end{array}\right|$ & $\begin{array}{c}34 \\
24 \\
24\end{array}$ & $\begin{array}{l}14 \\
15 \\
\end{array}$ & 19 & 52 & 69 & $A p$ & & th & Artemi, Poly-Arte \\
\hline Betonica officinalis $\mathrm{L}$. & 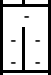 & & & & 1 & & -1 & & & 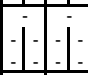 & & $i_{2}^{2}$ & & & & & & & & -1 & & 1 & $i$ & & $\mid \begin{array}{l}1 \\
1 \\
\end{array}$ & i & $\begin{array}{l}2 \\
3 \\
\end{array}$ & 1 & 3 & 7 & Ns & & $\mathrm{h}$ & \begin{tabular}{|l|}
$\begin{array}{l}\text { Trif-Ger, Fest-Brom, Quer } \\
\text { Fage }\end{array}$ \\
\end{tabular} \\
\hline $\begin{array}{l}\text { Botriochloa } \\
\text { ischaemum (L.) Keng }\end{array}$ & 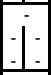 & & -1 & & \begin{tabular}{|c|c|c}
2 & 1 \\
2 & 1 & 1 \\
\end{tabular} & & $\therefore:$ & -1 & & $-i-$ & & $-1-$ & $\left.2\right|^{2}-x-3 \mid$ & & & & -1 & & & ${ }_{2}^{2} \mid \frac{1}{1}$ & & $i$ & $i$ & & $\left|\begin{array}{l}6 \\
4\end{array}\right|$ & $\begin{array}{l}10 \\
13\end{array}$ & $\begin{array}{l}9 \\
6 \\
\end{array}$ & 11 & 25 & 41 & Ns & & $\mathrm{h}$ & Fest-Brom \\
\hline $\begin{array}{l}\text { Brassica campestris } \\
\text { L. }\end{array}$ & 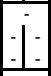 & & is & & $\therefore$ & & $::$ & $: 1:$ & & $-1:$ & & 1 & $: 1:$ & & i] & & -1 & 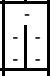 & & -1 & & : & $i$ & & \begin{tabular}{|l|} 
\\
4
\end{tabular} & il & $\dot{4}$ & 4 & 5 & 5 & Ar & C-Asiatic & $\mathrm{t}$ & Stel medi \\
\hline Brassica napus $\mathrm{L}$. & $i$ & & $-1-1$ & & $1-1$ & & $\therefore$ & & & : & & & & & & & & & & & & -1 & 1 & & $i$ & $: 1$ & $i$ & 1 & 1 & 1 & Eg & $\begin{array}{l}\text { s-European- } \\
\text { anthrop.? }\end{array}$ & $\mathrm{t}$ & Stel medi \\
\hline $\begin{array}{l}\begin{array}{l}\text { Bromopsis erecta } \\
\text { (Huds.) Fourr. }\end{array} \\
\end{array}$ & $i:$ & & & & -1 & & & 31 & & 1 & & & & & & & & & & & & & $j$ & 1 & : & 3 & - & 1 & 2 & 5 & Ns & & $\mathrm{h}$ & Fest-Brom \\
\hline $\begin{array}{l}\text { Bromopsis inermis } \\
\text { (Leyss.) Holub }\end{array}$ & \begin{tabular}{|l|}
3 \\
-2 \\
\end{tabular} & \begin{tabular}{|l|l|l}
1 & 2 \\
3 & 3 \\
\end{tabular} & 1 & & $-3_{3}^{2}$ & 1 & \begin{tabular}{|l|l|l|} 
& 3 \\
3 & 2 \\
3 & 3 \\
\end{tabular} & \begin{tabular}{|l|l|} 
& 3 \\
2 & 3 \\
\end{tabular} & & $-y_{3}^{1}$ & 12 & -2 & $\left.\right|_{-1} ^{2}$ & & & & & & & & - & 212 & $a_{3}^{2}$ & & $\left|\begin{array}{l}18 \\
21\end{array}\right|$ & $\begin{array}{l}15 \\
28 \\
\end{array}$ & $\begin{array}{l}34 \\
52 \\
\end{array}$ & 22 & 70 & 151 & $\mathrm{Ha}$ & & hg & \begin{tabular}{|l|}
$\begin{array}{l}\text { Gali veri, Moli-Arrh, Fest- } \\
\text { Brom, Agro int-rep }\end{array}$ \\
\end{tabular} \\
\hline $\begin{array}{l}\text { Bromopsis riparia } \\
\text { (Rehmann) Holub }\end{array}$ & -1 & & -1 & & \begin{tabular}{|l|l|}
2 & 2 \\
2 & - \\
\end{tabular} & & $1-1$ & & & 1 & & 2 & & & & & & & & & & -1 & $-1-$ & & \begin{tabular}{|l|}
3 \\
1
\end{tabular} & \begin{tabular}{l|}
2 \\
2
\end{tabular} & \begin{tabular}{|l}
6 \\
2 \\
\end{tabular} & 4 & 6 & 12 & Ns & & g & Fest-Brom \\
\hline Bromus squarrosus $\mathrm{L}$. & $-1:$ & & & & -1 & & & & & $i$ & & & & & & & & & & & & & j. & & \begin{tabular}{|l|}
1 \\
1 \\
\end{tabular} & i & $\begin{array}{l}1 \\
1 \\
\end{array}$ & 3 & 3 & 3 & Ar? & \begin{tabular}{|l|}
$\begin{array}{l}\text { Mediterr- } \\
\text { continent- } \\
\text { Eurasiatic }\end{array}$ \\
\end{tabular} & $\mathrm{t}$ & \begin{tabular}{|l|} 
Poly-Arte, [Fest-Brom], \\
Fest vagi, Stel medi, Agro \\
int-rep
\end{tabular} \\
\hline $\begin{array}{l}\text { Buglossoides } \\
\text { arvensis (L.) }\end{array}$ & -1. & -1 & -1 & & 2 & & -1 & & & 1 & & & -1 & & & & & & & & & 1 & $i_{1}$ & $2 \begin{array}{l}2 \\
2 \\
5\end{array}$ & \begin{tabular}{|l|}
2 \\
2 \\
3
\end{tabular} & $\begin{array}{r}3 \\
8 \\
\end{array}$ & $\begin{array}{l}2 \\
4 \\
\end{array}$ & 8 & 14 & 20 & Ar & \begin{tabular}{|l|}
$\begin{array}{l}\text { Mediterr-Iranian- } \\
\text { Turanian }\end{array}$ \\
\end{tabular} & $\mathrm{t}$ & Stel medi, [Poly-Artemi] \\
\hline
\end{tabular}




\begin{tabular}{|c|c|c|c|c|c|c|c|c|c|c|c|c|c|c|c|c|c|c|c|c|c|c|c|c|c|c|c|c|c|c|}
\hline & 1 & 2 & 3 & 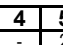 & \begin{tabular}{l|l|}
5 & 6 \\
2 & -
\end{tabular} & 7 & $8 \mid$ & 9 & \begin{tabular}{|l|l|}
10 & 11 \\
\end{tabular} & \begin{tabular}{|l|l|}
12 & 13 \\
\end{tabular} & \begin{tabular}{l|l}
3 & 14 \\
\end{tabular} & 15 & 16 & \begin{tabular}{|l|l|}
17 & 1 \\
\end{tabular} & \begin{tabular}{l|l|}
18 & 19 \\
& -
\end{tabular} & 20 & 21 & 22 & \begin{tabular}{|l|l|}
23 & 24 \\
\end{tabular} & 25 & $\frac{26}{2}$ & & 27 & 28 & 29 & 30 & 31 & 32 & 33 & 34 \\
\hline $\begin{array}{l}\text { Calamagrostis } \\
\text { epigeios (L.) Roth }\end{array}$ & $\therefore$ & & & & $\mid \begin{array}{ll}2 & 1 \\
2 & 1 \\
2 & -\end{array}$ & $\because:-1$ & $1=$ & & i. & & & & & & $\left|\begin{array}{l}-1 \\
2\end{array}\right|$ & & & & 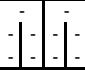 & -1. & \begin{tabular}{|l|l|l|}
1 & 3 \\
3 & 5 \\
\end{tabular} & & \begin{tabular}{|l|l|l|}
1 & 5 \\
0 & 10 \\
\end{tabular} & 7 & 14 & 25 & $\mathrm{Ha}$ & & $\mathrm{hg}$ & $\begin{array}{l}\text { Agro int-rep, Fest vagi, } \\
\text { Gali veri, Moli-Arrh }\end{array}$ \\
\hline $\begin{array}{l}\text { Camelina microcarpa } \\
\text { Andrz. }\end{array}$ & $i$ & & & - & -1 & $\begin{array}{ll}1 \\
-1 \\
-1\end{array}$ & 1 & & is & & & & & & & & & & & $\begin{array}{lll}1 & - \\
2 & - & 0 \\
\end{array}$ & & & \begin{tabular}{|l|l|l}
6 & 2 \\
9 & 8 \\
\end{tabular} & 11 & 21 & 28 & Ar? & $\begin{array}{l}\text { Mediterr- } \\
\text { Turanian }\end{array}$ & th & Stel medi, [Poly-Artemi] \\
\hline $\begin{array}{l}\text { Campanula } \\
\text { bononiensis } \mathrm{L} .\end{array}$ & $i$ & & & & & . & 1 & & & & & & & & 1 & & & & & \begin{tabular}{|l|l|l|} 
& - & - \\
2 & - & - \\
2 & 2 \\
\end{tabular} & \begin{tabular}{|l|l|}
1 & 4 \\
3 & 5 \\
\end{tabular} & & \begin{tabular}{l|l|}
1 & 5 \\
4 & 8
\end{tabular} & 5 & 13 & 18 & Ns & & h & Fest-Brom, Trif-Gera \\
\hline Campanula sibirica L. & $-i$ & & & & & i & & & & & & & & & & & & & 1 & -1. & \begin{tabular}{l|l|l|l|} 
& 2 \\
7 & 9
\end{tabular} & & \begin{tabular}{l|l|}
5 & 12 \\
3 & 9
\end{tabular} & 13 & 34 & 52 & Ns & & $\mathrm{h}$ & Fest-Brom \\
\hline Cannabis sativa $\mathrm{L}$. & $-i$ & & & & & 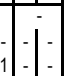 & & & & & & & & & & & & & $j$ & \begin{tabular}{r|r}
1 \\
-1 \\
-1 \\
\end{tabular} & & & & 3 & 4 & 4 & Ke & E-Asiatic & $t$ & \begin{tabular}{|l}
$\begin{array}{l}\text { Stel medi, Artemi, [Fest } \\
\text { vagi] }\end{array}$ \\
\end{tabular} \\
\hline $\begin{array}{l}\text { Capsella bursa- } \\
\text { pastoris (L.) Medik. }\end{array}$ & -1 & & & & & $i$ & 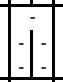 & & i. & $\begin{array}{l}1 \\
2 \\
1\end{array}$ & & & & & \begin{tabular}{ll|l|l|}
11 & 2 \\
1
\end{tabular} & & & & $\begin{array}{ll}1 \\
2 \\
1 \\
-1\end{array}$ & \begin{tabular}{|c|c|} 
& 2 \\
2 & 1 \\
-2 \\
\end{tabular} & \begin{tabular}{|l|l|} 
& 5 \\
6 & 5 \\
6 & 8 \\
\end{tabular} & & \begin{tabular}{|c|c|}
6 & 5 \\
2 & 12 \\
\end{tabular} & 16 & 32 & 46 & Ar & unknown origin & th & Stel medi, Plan majo \\
\hline $\begin{array}{l}\text { Caragana frutex (L.) } \\
\text { K.Koch }\end{array}$ & -1 & & & & 工 & -1 & $j$ & & 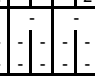 & - & & & & $\perp$ & ב & & -1 & & i & - & $-j$ & & 1 & 1 & 1 & 1 & Ns & & nf & Fest-Brom \\
\hline $\begin{array}{l}\text { Caragana } \\
\text { arborescens Lam. }\end{array}$ & -1 & & & & & -1 & -1 & & 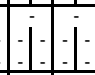 & 1 & & & & 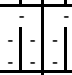 & 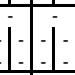 & & & & . & -1. & $-i$ & & $i$ & 1 & 1 & 1 & $E g$ & WC-Asiatic & nf & Robin \\
\hline $\begin{array}{l}\text { Cardaria draba (L.) } \\
\text { Desv. }\end{array}$ & -1. & & & & & 1. & \begin{tabular}{|c|c|} 
& 2 \\
2 & 2 \\
2 & 1 \\
\end{tabular} & & i. & & & & & & & & & & 开 & $-i$ & & \begin{tabular}{|l|l}
1 & 2 \\
1 & 2 \\
\end{tabular} & \begin{tabular}{l|l|}
2 & 2 \\
2 & 1 \\
\end{tabular} & 1 & 5 & 9 & $\mathrm{Ke}$ & E-Mediterr & hg & Agro int-rep \\
\hline $\begin{array}{l}\text { Carduus acanthoides } \\
\text { L. }\end{array}$ & -1 & & & & 1 & -1. & -1 & & 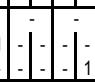 & & & & & & & & & & & 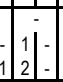 & \begin{tabular}{|l|l|} 
& 3 \\
3 & 3 \\
4 & 3 \\
\end{tabular} & \begin{tabular}{|l|l}
3 & 4 \\
3 & 7 \\
\end{tabular} & \begin{tabular}{|l|l|}
1 & 5 \\
4 & 3 \\
7 & 3 \\
\end{tabular} & 10 & 16 & 22 & $\mathrm{Ar}$ & $\begin{array}{l}\text { sub-Mediterr- } \\
\text { sub-Atlantic- } \\
\text { European }\end{array}$ & th & $\begin{array}{l}\text { Artemi, Agro int-rep, Fest- } \\
\text { Brom }\end{array}$ \\
\hline Carduus nutans $\mathrm{L}$. & \begin{tabular}{|c|}
1 \\
1 \\
1 \\
\end{tabular} & & & & & \begin{tabular}{|c|c|} 
& 1 \\
1 & - \\
1 & - \\
\end{tabular} & 1 & & $-\begin{array}{c}-1 \\
-1 \\
1\end{array}$ & $\begin{array}{c}1 \\
-1 \\
1\end{array}$ & & & & $\begin{array}{ccc}-1 & 1 \\
& -1 & 1\end{array}$ & \begin{tabular}{l|l|}
$j$ & 1 \\
\end{tabular} & & & & & \begin{tabular}{|l|l|} 
& 1 \\
1 & 1 \\
1 & 1 \\
\end{tabular} & \begin{tabular}{|l|l|}
\multicolumn{1}{|c|}{9} \\
11 & 7 \\
7 & 6 \\
\end{tabular} & & \begin{tabular}{l|l|}
10 & 7 \\
1 & 6 \\
\end{tabular} & 15 & 40 & 41 & Ar & \begin{tabular}{|l|} 
sub-Atlantic- \\
boreal-European
\end{tabular} & th & Artemi \\
\hline Carex hirta $L$. & $\therefore:$ & & & & & $i$ & -1 & & i. & 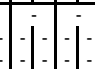 & & & & 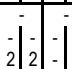 & & & & & -1 & i. & & & \begin{tabular}{l|l|} 
& - \\
\end{tabular} & 2 & 4 & 6 & $\mathrm{Ha}$ & & g & Moli-Arrh, Agr int-rep, \\
\hline $\begin{array}{l}\text { Carex melanosta- } \\
\text { chya M.Bieb.ex Willd. }\end{array}$ & -1 & & & & . & -1 & & & 1 & -1 & & & & & & & & & & $-1-$ & $\begin{array}{l}1 \\
2 \\
\end{array}$ & $\begin{array}{l}1 \\
2 \\
\end{array}$ & \begin{tabular}{l|l|}
2 \\
- & 5 \\
\end{tabular} & 2 & 4 & 8 & Ns & & $\mathrm{g}$ & $\begin{array}{l}\text { Gali veri, Moli-Arrh, Fest- } \\
\text { Brom, Alth offi }\end{array}$ \\
\hline Carex michelii Host & $i:$ & & & & & $-1:$ & 1.1 & & & & & & & & & & & & . & 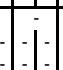 & & & $-\left|\begin{array}{l}1 \\
2\end{array}\right|$ & 1 & 2 & 3 & Ns & & $\mathrm{h}$ & Fest-Brom, Quer-Fage \\
\hline $\begin{array}{l}\text { Carex praecox } \\
\text { Schreb. } \\
\end{array}$ & \begin{tabular}{|l|l|} 
& 1 \\
3 & 2 \\
3 & 3 \\
\end{tabular} & \begin{tabular}{|l|l|}
2 & 2 \\
2 & 2 \\
& 2 \\
\end{tabular} & \begin{tabular}{|c|c|}
1 & \\
2 & 3 \\
2 & 3
\end{tabular} & & -11 & & & & $i$ & 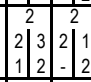 & $\begin{array}{l}1 \\
2 \\
\end{array}$ & & & 1. & & & & $3]$ & \begin{tabular}{|l|l|l|l|}
2 & 2 & 2 & 2 \\
& 2 & -1 & \\
\end{tabular} & \begin{tabular}{lll|}
2 & 3 & 2 \\
& 2 & - \\
\end{tabular} & \begin{tabular}{|l|l|}
9 & 14 \\
9 & 13 \\
\end{tabular} & $\begin{array}{ll}14 & 20 \\
13 & 19 \\
\end{array}$ & \begin{tabular}{|l|l|}
0 & 29 \\
& 25 \\
\end{tabular} & 16 & 52 & 102 & $\mathrm{Ha}$ & & hg & \begin{tabular}{|l|}
$\begin{array}{l}\text { Gali veri, Moli-Arrh, Fest-Brom, } \\
\text { Fest vagi, Agro int-rep }\end{array}$ \\
\end{tabular} \\
\hline Carex spicata Huds. & $\therefore$ & & & & & $i$ & & & $i$ & . & & & & & & & & & $i$ & 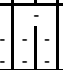 & $\therefore:$ & & $-i_{2}$ & 2 & 2 & 2 & $\mathrm{Ha}$ & & $\mathrm{h}$ & \begin{tabular}{|l} 
Moli-Arrh, Quer-Fage, \\
Robin, Gali veri
\end{tabular} \\
\hline $\begin{array}{l}\text { Carex stenophylla } \\
\text { Wahlenb. }\end{array}$ & 2 & & &. .1. & -1 & - & & & $\int_{1}^{1}$ & - & & & & & $-i-$ & $\mid \begin{array}{r}2 \\
3 \\
3\end{array}$ & & \begin{tabular}{|r|r} 
& 2 \\
3 \\
\end{tabular} & is & $i$ & \begin{tabular}{|l|l|} 
& 3 \\
4 & 2 \\
3 & 1 \\
\end{tabular} & $\begin{array}{ll}2 & 11 \\
1 & 7 \\
\end{array}$ & \begin{tabular}{l|l|}
6 & 3 \\
& 3 \\
\end{tabular} & 7 & 13 & 28 & \begin{tabular}{|c|}
$N s$ \\
$(* * *)$
\end{tabular} & & $\mathrm{g}$ & Fest-Brom \\
\hline $\begin{array}{l}\text { Carex supina Willd. ex } \\
\text { Wahlenb. }\end{array}$ & -1 & -12 & & -1 & 1. &. & 1 & & \begin{tabular}{|l|l|}
2 & 2 \\
& - \\
\end{tabular} & 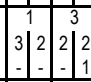 & & & & & & & & & $-\begin{array}{l}2 \\
1 \\
\end{array}$ & $\begin{array}{lll}2 & -1 \\
& - & 0\end{array}$ & \begin{tabular}{|l|l|}
4 & 5 \\
- & 3 \\
\end{tabular} & $\begin{array}{ll}5 & 9 \\
3 & 9 \\
\end{array}$ & \begin{tabular}{l|c|}
9 & 10 \\
& 4 \\
\end{tabular} & 8 & 16 & 30 & \begin{tabular}{|c|}
$N s$ \\
$(* * *)$ \\
\end{tabular} & & hg & Fest-Brom \\
\hline $\begin{array}{l}\text { Carlina biebersteinii } \\
\text { Bernh. ex Hornem. }\end{array}$ & -1 & & -1 & $1:$ & & $1:$ & 1 & & 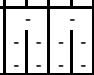 & -1. & $-\begin{array}{c}1 \\
1 \\
1\end{array} \mid$ & $-\begin{array}{l}2 \\
2 \\
\end{array}$ & & & & -1 & & & $i$ & 1 & \begin{tabular}{|l|l|}
2 & 4 \\
2 & 2 \\
\end{tabular} & \begin{tabular}{l|l}
4 & 3 \\
2 & 2 \\
\end{tabular} & \begin{tabular}{l|l|}
3 & 5 \\
2 & 3 \\
\end{tabular} & 4 & 11 & 14 & Ns & & ht & Fest-Brom, Fest vagi \\
\hline $\begin{array}{l}\text { Centaurea adpressa } \\
\text { Ledeb. }\end{array}$ & \begin{tabular}{|l|l|}
1 & 2 \\
2 & 2 \\
\end{tabular} & & \begin{tabular}{|l|l|}
- & 1 \\
2 & 2 \\
\end{tabular} & & $2 \begin{array}{l}1 \\
2 \\
\end{array}$ & $:-1:$ & $-1:-1$ & -1 & $\therefore:$ & 1 & & & & 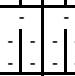 & $\begin{array}{l}2 \\
2\end{array} \mid$ & $\begin{array}{lll} & -1 \\
1 & & 1 \\
\end{array}$ & & 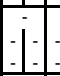 & 1 & -1. & \begin{tabular}{|l|l|}
2 & 4 \\
7 & 5 \\
\end{tabular} & \begin{tabular}{l|l}
4 & 3 \\
5 & 14 \\
\end{tabular} & \begin{tabular}{l|l|}
3 & 5 \\
4 & 8 \\
\end{tabular} & 7 & 19 & 31 & Ns & & $\mathrm{h}$ & \begin{tabular}{|l|}
$\begin{array}{l}\text { Fest-Brom, Fest vagi, Gali } \\
\text { veri }\end{array}$ \\
\end{tabular} \\
\hline $\begin{array}{l}\text { Centaurea apiculata } \\
\text { Ledeb. } \\
\end{array}$ & 1 & & $1:$ & : & $1:$ & $-1-1$ & 1 & & $i$ & \begin{tabular}{r|r}
$-i$ & -1 \\
& 1 \\
\end{tabular} & & -1. & & -1 & -1 & 1 & -1 & & 1 & -1. & \begin{tabular}{|l|l} 
& 1 \\
1 & 1 \\
\end{tabular} & & \begin{tabular}{l|l|}
1 \\
1 & 2 \\
\end{tabular} & 1 & 3 & 4 & Ns & & $\mathrm{h}$ & Fest-Brom \\
\hline $\begin{array}{l}\text { Centaurea bibersteinii } \\
\text { DC. }\end{array}$ & $i:$ & $\because:$ & $1:$ & 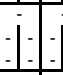 & 10 & . & 1. & $-1-$ & -10.1 & i. & 1 & $\therefore:$ & -1 & -1.1 & -1. & $\because$ &. & -1. & 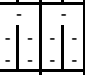 & -1. & $-i_{1}^{1}$ & & 1 & 1 & 1 & 1 & Ns & & $\mathrm{h}$ & Fest-Brom \\
\hline \begin{tabular}{|c|} 
Centaurea stoebe $\mathrm{L}$. \\
.
\end{tabular} & -1. & & -1 & i & & \begin{tabular}{|l|l|l|l|l|} 
& 2 \\
2 & 2 \\
-2 & 1 \\
\end{tabular} & 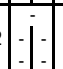 & & 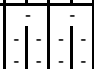 & 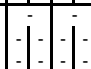 & $i$ & 1 & & 1 & -1 & -1 & 1. & 1 & $-1-$ & -1 & $\left.1\right|^{1} 1$ & $\begin{array}{ll}1 & 2 \\
2 & ?\end{array}$ & $2\left|\begin{array}{l}2 \\
2\end{array}\right|$ & 2 & 5 & 8 & $\mathrm{Ha}$ & & $\mathrm{h}$ & Fest-Brom, Artemi \\
\hline
\end{tabular}




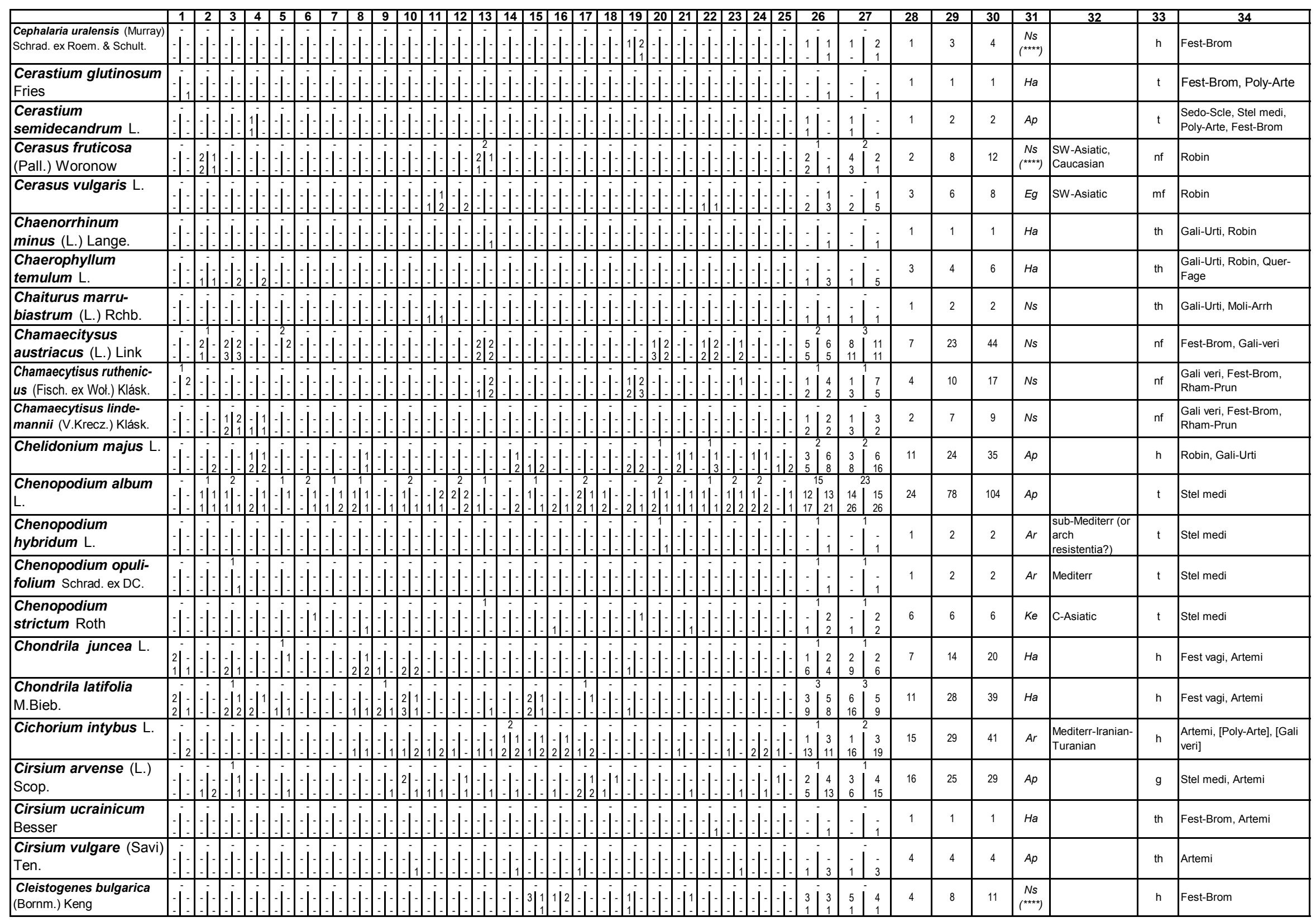




\begin{tabular}{|c|c|c|c|c|c|c|c|c|c|c|c|c|c|c|c|c|c|c|c|c|c|c|c|c|c|c|c|c|c|c|c|c|c|}
\hline & 1 & 2 & 3 & 4 & \begin{tabular}{c|c}
5 & 6 \\
\end{tabular} & 7 & 8 & 9 & 1 & $0 \mid 11$ & 112 & & \begin{tabular}{|l|l|}
14 & 15 \\
\end{tabular} & \begin{tabular}{l|l}
15 & 16 \\
\end{tabular} & \begin{tabular}{l|l|}
6 & 17 \\
\end{tabular} & 18 & 19 & 20 & \begin{tabular}{l|l}
21 & 22 \\
\end{tabular} & 223 & 24 & 25 & & $\frac{26}{1.6}$ & & $\frac{27}{2}$ & 28 & 29 & 30 & 31 & 32 & 33 & 34 \\
\hline Conium maculatum $\mathrm{L}$. & -1 & & & & & & & & & & & & & & & & & & & & & \begin{tabular}{|c|}
2 \\
-1 \\
1
\end{tabular} & & $\left|\begin{array}{l}1 \\
3\end{array}\right|$ & & $\mid \begin{array}{l}2 \\
4\end{array}$ & 3 & 7 & 10 & Ar & \begin{tabular}{|l|} 
Mediterr-Iranian- \\
Turanian
\end{tabular} & th & Artemi, Stel medi \\
\hline $\begin{array}{l}\text { Consolida orientalis (J.Gay } \\
\text { ex Gren. \& Godr.) Schroed. }\end{array}$ & $\mid-1$ & & . & & -1 & & & & & & & 1 & $\because$ & & & & & & & 1 & & $-1-$ & & & & 1: & 1 & 1 & 1 & Ke & Mediter & $\mathrm{t}$ & Stel medi \\
\hline \begin{tabular}{|l|}
$\begin{array}{l}\text { Consolida paniculata } \\
\text { (Host) Schur }\end{array}$ \\
\end{tabular} & 1 & & & & & & & & & & & & & & & & & & & & & -1 & & $\begin{array}{c}5 \\
12\end{array} \mid$ & & $\mid \begin{array}{l}5 \\
20\end{array}$ & 16 & 36 & 54 & $A p$ & & $\mathrm{t}$ & Poly-Arte, Stel medi \\
\hline $\begin{array}{l}\text { Consolida regalis S.F. } \\
\text { Gray }\end{array}$ &. & & & & & & & & & & & & & & & & $-i$ & & .1 & & & $-i$ & & $\left|\begin{array}{l}1 \\
1 \\
1\end{array}\right|$ & & $\mid \begin{array}{l}1 \\
1\end{array}$ & 1 & 4 & 4 & Ke & Mediter & $\mathrm{t}$ & Stel medi \\
\hline $\begin{array}{l}\text { Convolvulus arvensis } \\
\text { L. }\end{array}$ & \begin{tabular}{|l|l|l}
2 & 2 \\
2 & 2 \\
\end{tabular} & & 2 & & & & $\begin{array}{l}2 \\
2 \\
2\end{array}$ & & 3. & & & & & & & \begin{tabular}{|l|l|l|l|l|l|}
2 & 2 \\
1 & 2 & 2 \\
\end{tabular} & & & & 21 & & $\begin{array}{rl}-i & 1 \\
2 & 2 \\
2\end{array}$ & & \begin{tabular}{|l|}
20 \\
23 \\
23 \\
\end{tabular} & & $\begin{array}{l}36 \\
36 \\
43 \\
\end{array}$ & 24 & 106 & 198 & $A p$ & & hg & Agro int-rep, Stel medi \\
\hline $\begin{array}{l}\text { Conyza canadensis } \\
\text { (L.) Cronq. }\end{array}$ & $-i$ & & & & & 12 & I & & & & & & & & & \begin{tabular}{|l|l|l|l|l|l|l|}
2 & 1 \\
2 & 2 \\
\end{tabular} & & & 2 & $-\begin{array}{l}2 \\
1\end{array} \mid$ & \begin{tabular}{|c|c|} 
& 1 \\
3 & 1 \\
2 & 2 \\
\end{tabular} & $\begin{array}{ll}-j \\
2\end{array}$ & & \begin{tabular}{|l|}
12 \\
12 \\
21 \\
\end{tabular} & $\begin{array}{l}15 \\
38 \\
\end{array}$ & \begin{tabular}{|l}
14 \\
29 \\
29
\end{tabular} & 24 & 76 & 114 & Ke & North American & th & Stel medi, [Fest vagi] \\
\hline Coronilla varia $\mathrm{L}$. & -12 & & & & & & 2 & & 2 & & & & & & & & & & & & & $: y^{1}$ & & $\begin{array}{l}5 \\
5\end{array}$ & & $\mid \begin{array}{l}6 \\
9\end{array}$ & 7 & 15 & 24 & $\mathrm{Ha}$ & & $\mathrm{h}$ & $\begin{array}{l}\begin{array}{l}\text { Fest-Brom, Gali veri, Fest } \\
\text { vagi }\end{array} \\
\end{array}$ \\
\hline $\begin{array}{l}\text { Corydalis solida } \\
\text { Clairv. }\end{array}$ & 1 & & $-i$ & & & $i$ & -1 - & & & & $\because$ & 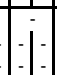 & $j$ & & & . & -1 & & 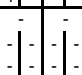 & -1 & & $i$ & & $i \mid$ & & $I_{2}$ & 1 & 1 & 2 & Ns & & $\mathrm{g}$ & Quer-Fage \\
\hline $\begin{array}{l}\text { Cotinus coggygria } \\
\text { Scop. }\end{array}$ & $\therefore:$ & & 中 & & & $1-$ & ـ & & & &. & $i$ & $i$ & & & $: 1$ & 1 & & &. & & $i$ & & i & & $\mid i$ & 1 & 2 & 2 & Ke & $\begin{array}{l}\text { S-European- } \\
\text { SWAsiatic }\end{array}$ & nf & Robin \\
\hline \begin{tabular}{|l|} 
Crataegus mono-gyna \\
Jacq. aggr. \\
\end{tabular} & -1 & & i & & & -. & & & & & . & $i$ & -1 & & & -1 & & & -1 & & & $-1:$ & & \begin{tabular}{|l|}
1 \\
3 \\
\end{tabular} & & \begin{tabular}{|l}
1 \\
3 \\
\end{tabular} & 4 & 8 & 8 & Ns & & $\mathrm{nf}$ & Rham-Prun \\
\hline Crataegus sp. & -1. & & 1.1 & & & & & & & & & i & $i$ & & & & & & $i$ & - & 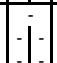 & $-1:$ & & 1 & & 1 & 1 & 2 & 2 & Ns & & $\mathrm{nf}$ & Rham-Prun \\
\hline Crepis biennis $\mathrm{L}$. & -1 & & & & & & & & & & & & & & & & & & & & & $i$ & & 1 & & $\mid \begin{array}{l}1 \\
1\end{array}$ & 1 & 2 & 2 & $\mathrm{Ha}$ & & $\mathrm{h}$ & Moli-Arrh \\
\hline \begin{tabular}{|l|} 
Crepis ramosissima \\
D`Urv. \\
\end{tabular} & $1:$ & & -1 & & & & & & & & & 1 & - & & & & & & & & & 1 & & $\begin{array}{l}3 \\
5\end{array}$ & & \begin{tabular}{|l}
3 \\
5
\end{tabular} & 8 & 13 & 13 & $\mathrm{Ha}$ & & $\mathrm{t}$ & Fest vagi, Stel medi \\
\hline $\begin{array}{l}\text { Crepis rhoeadifolia } \\
\text { M.Bieb. } \\
\end{array}$ & $1:$ & & $1-1$ & & & & & & & & & -1 & & & & 11 & & & -1 & & & -1. & & 2 & & $\left.\right|_{2}$ & 5 & 6 & 7 & $A p$ & & $\mathrm{t}$ & Fest vagi, Artemi \\
\hline $\begin{array}{l}\text { Crepis tectorum } \mathrm{L} \text {. } \\
\text {. }\end{array}$ & -1 & & & & & & i. & & & & & 1 & & & & -1 & & & -1 & 1.1. & -1 & 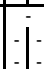 & & i & & i & 1 & 2 & 2 & $A p$ & & $\mathrm{t}$ & Stel medi \\
\hline $\begin{array}{l}\text { Cuscuta campestris } \\
\text { Yunck. } \\
\end{array}$ & $\therefore:$ & & -1 & & & & -1 & & & & & -1 & 1 & & & -1 & -1 & & $-i$ & -1. & -1 & $: 1:$ & $i_{2}$ & & i & $:$ & 2 & 2 & 2 & Ke & North American & $\mathrm{t}$ & Stel medi \\
\hline $\begin{array}{l}\text { Cynoglossum } \\
\text { officinale } \mathrm{L} .\end{array}$ & -1. & & i] & & & $-1 \begin{array}{l}1 \\
1\end{array}$ & & & & & & & & & & & & & 2 & & & \begin{tabular}{|r|r|} 
& 1 \\
1 & 2 \\
1 & 1 \\
\end{tabular} & & $\begin{array}{c}6 \\
11 \\
\end{array}$ & \begin{tabular}{|c}
4 \\
10 \\
\end{tabular} & $\mid \begin{array}{l}7 \\
11 \\
\end{array}$ & 16 & 34 & 38 & Ar & $\begin{array}{l}\text { Eurasiatic- } \\
\text { continent }\end{array}$ & $\mathrm{h}$ & Artemi \\
\hline Dactylis glomerata L. & 1 & & & & & & & & & & & & & & & & & & i) & & & $1-1$ & & $\begin{array}{l}1 \\
4 \\
\end{array}$ & & $\mid \begin{array}{l}2 \\
2 \\
\end{array}$ & 5 & 10 & 18 & $A p$ & & $\mathrm{hg}$ & Moli-Arrh \\
\hline Datura stramonium L. & $i$ & & & & & & & & & & 1.1 & $i$ & $\because$ & & & T & 1.1 & & $\because: 1$ & $i$ & $i$ & -1 & & 1 & & i & 1 & 1 & 1 & Ar & SE-Asiatic & $\mathrm{t}$ & Stel medi \\
\hline Daucus carota L. & & & -1 & & il 1 i & $\because:$ & & & & & 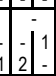 & 1 & $-i_{2}^{2}-j$ & {$\left[\begin{array}{l}1 \\
2\end{array}\right]_{2}^{2}$} & & & & -1 & $i=1$ & & 1] & $i$. & & $\begin{array}{c}4 \\
13 \\
\end{array}$ & & $\mid \begin{array}{l}6 \\
20 \\
\end{array}$ & 16 & 27 & 41 & $A p$ & & $\mathrm{~h}$ & Moli-Arrh, Artemi \\
\hline \begin{tabular}{|l|} 
Descurainia sophia \\
(L.) Webb ex Prantl
\end{tabular} & $\mid-1$ & & 1 & & -1 & -1 & & & & & 1 & & il & & & $1]$ & -1 & 1 & it & & $-\begin{array}{ll}1 \\
2 \\
1\end{array} \mid$ & 11. & & $\begin{array}{l}3 \\
7 \\
\end{array}$ & $\begin{array}{r}3 \\
12 \\
\end{array}$ & $\begin{array}{l}3 \\
7 \\
\end{array}$ & 15 & 26 & 31 & Ar & Iranian-Turanian & $t$ & Stel medi \\
\hline \begin{tabular}{|l|} 
Dianthus campestris \\
M.Bieb. \\
\end{tabular} & \begin{tabular}{|l|l}
2 & 2 \\
2 & 1 \\
\end{tabular} & & -1 & & \begin{tabular}{|l|l|l|l|l}
2 & 2 & 2 & 2 \\
2 & 2 & 2 \\
1 & 1 & 1 \\
\end{tabular} & \begin{tabular}{l|lll}
2 & 2 & 2 \\
1 & 2 & 2 \\
& 2 & 1 \\
\end{tabular} & $\left.\begin{array}{l}1 \\
1\end{array}\right] 2$ & & & & $1-1$ & & \begin{tabular}{|lll} 
& 2 & 2 \\
\end{tabular} & & & & -1 & & -1 & & -1 & & & \begin{tabular}{|l|}
5 \\
5 \\
\end{tabular} & $\begin{array}{c}12 \\
7 \\
\end{array}$ & \begin{tabular}{|c|c}
10 \\
6
\end{tabular} & 7 & 24 & 41 & Ns & & $\mathrm{h}$ & Fest-Brom \\
\hline $\begin{array}{l}\text { Dianthus carbonatus } \\
\text { M.Bieb. }\end{array}$ & . & $\mid-1$ & 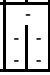 & & $-1-1$. & $\because 1$ & : & -1 & & & 1 & 1 & 过: & $1:$ & 8 & 1 & $\left.$\begin{tabular}{|l|l|l|l|}
2 & 2 \\
2
\end{tabular}\right|$^{2}$ & \begin{tabular}{ll|}
1 & 1 \\
& 1 \\
\end{tabular} & \begin{tabular}{l|l|l|l}
2 & 1 & 1 \\
1 & 2 & 2
\end{tabular} & : & $\therefore$ & $1-1$ & & $\begin{array}{l}3 \\
1 \\
\end{array}$ & $\begin{array}{l}4 \\
4 \\
\end{array}$ & \begin{tabular}{|l}
5 \\
1 \\
\end{tabular} & 4 & 10 & 15 & Ns & & $\mathrm{h}$ & Fest-Brom \\
\hline
\end{tabular}




\begin{tabular}{|c|c|c|c|c|c|c|c|c|c|c|c|c|c|c|c|c|c|c|c|c|c|c|c|c|c|c|c|c|c|c|c|c|}
\hline & \begin{tabular}{|l|l|}
1 & 2 \\
\end{tabular} & 3 & \begin{tabular}{|l|l|}
4 & \\
\end{tabular} & 5 & 6 & 7 & 8 & 9 & 10 & 11 & 12 & & \begin{tabular}{l|l}
14 & 15 \\
\end{tabular} & \begin{tabular}{|l|}
516 \\
\end{tabular} & \begin{tabular}{|l|l|}
17 & 1 \\
\end{tabular} & \begin{tabular}{l|l}
18 & 19 \\
\end{tabular} & \begin{tabular}{l|l|}
9 & 20 \\
\end{tabular} & \begin{tabular}{|l|l|}
21 & 2 \\
\end{tabular} & \begin{tabular}{l|l}
223 \\
2
\end{tabular} & \begin{tabular}{l|l}
324 \\
\end{tabular} & 425 & 26 & & 2 & 7 & 28 & 29 & 30 & 31 & 32 & 33 & 34 \\
\hline \begin{tabular}{|l|} 
Dianthus guttatus \\
M.Bieb. \\
\end{tabular} & -1. & & & & & & & & & & & & & & & $i$ & & & & 1.1 & & 1 & & 1 & & 1 & 1 & 1 & Ns & & $\mathrm{h}$ & Fest-Brom \\
\hline $\begin{array}{l}\text { Dianthus elongatus } \\
\text { C.A.Mey. }\end{array}$ & $1:-1$ & -1 & & 1 & & -1 &. & & . & & & & & & & {$\left[\begin{array}{ccc}-1 \\
-1\end{array}\right]$} & & $-1:$ & & 1 & & & 1 & 1 & 1 & 1 & 3 & 3 & Ns & & $\mathrm{h}$ & Fest-Brom \\
\hline $\begin{array}{l}\text { Dianthus membra- } \\
\text { naceus Borbás }\end{array}$ & 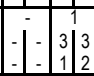 & \begin{tabular}{|l|l|} 
& 1 \\
1 & 1 \\
1 & 2 \\
\end{tabular} & & -1 & & & & & & & & & & & & -1 & & & & 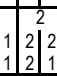 & i. . & & $\begin{array}{l}6 \\
5 \\
\end{array}$ & $\begin{array}{l}9 \\
9 \\
\end{array}$ & $\begin{array}{c}10 \\
7 \\
\end{array}$ & 7 & 25 & 39 & Ns & & $\mathrm{h}$ & Fest-Brom \\
\hline Draba nemorosa $\mathrm{L}$. & $\because$ & 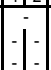 & & & & & & & & & & & & & & 2 & & ! & & & & & 5 & $\begin{array}{c}11 \\
9\end{array}$ & $\begin{array}{l}7 \\
7 \\
2\end{array}$ & 8 & 21 & 36 & Ns & & $\mathrm{t}$ & Fest-Brom, Poly-Arte \\
\hline Echium vulgare $\mathrm{L}$. & $-j$ & & & & & & & & & & & & & & & 1 & & & & & & 1 & & 1 & - & 3 & 3 & 3 & $A p$ & & th & Artemi \\
\hline $\begin{array}{l}\text { Echinochloa crus- } \\
\text { galli (L.) P.Beauv. }\end{array}$ & $i$ & $-\bar{i}$ & & & & & & & & & & & & & & $i$ & & & & & & -1 & $\dot{5}$ & & 5 & 7 & 9 & 9 & Ar & \begin{tabular}{|l|} 
Meditrr-Iranian- \\
Turanian-Indian- \\
Malay
\end{tabular} & $\mathrm{t}$ & Stel medi \\
\hline $\begin{array}{l}\text { Elisanthe noctiflora } \\
\text { (L.) Rupr. }\end{array}$ & 1 & -1 & & 1 & & & & & -1 & & & & & & & 1 & & & & & & & $i$ & : & $i$ & 1 & 1 & 1 & $A p$ & & $\mathrm{t}$ & Stel medi \\
\hline \begin{tabular}{|l|} 
Elisanthe viscosa (L.) \\
Rupr.
\end{tabular} & $1:$ & -1 & & 1 & & 1 12 . & T-1 & $\therefore-1$ & -1 & & & 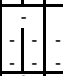 & & & & $i$ & t & T- & & 1 & 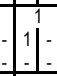 & \begin{tabular}{l|l}
5 \\
4 \\
3
\end{tabular} & 2 & $\begin{array}{l}6 \\
3 \\
\end{array}$ & $\begin{array}{l}3 \\
\end{array}$ & 5 & 14 & 19 & \begin{tabular}{|c|}
$N s$ \\
$(* * * *)$ \\
\end{tabular} & & $\mathrm{h}$ & Fest-Brom \\
\hline $\begin{array}{l}\text { Elytrigia intermedia } \\
\text { (Host) Nevski }\end{array}$ & 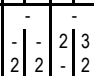 & $\begin{array}{ll}2 \\
2 \\
-2 \\
2\end{array}$ & & 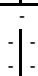 & & & & & & & & & & & & & & & & & & $\begin{array}{l}4 \\
3\end{array}$ & $\begin{array}{l}7 \\
9\end{array}$ & $\begin{array}{l}8 \\
4\end{array}$ & \begin{tabular}{|l|}
16 \\
18
\end{tabular} & 10 & 24 & 48 & $\mathrm{Ha}$ & & $\mathrm{hg}$ & $\begin{array}{l}\text { Gali veri, Fest-Brom, } \\
\text { Agro int-rep }\end{array}$ \\
\hline \begin{tabular}{l|} 
Elytrigia $x$ mucronata \\
(Opiz) Prokud.
\end{tabular} & \begin{tabular}{|c|c|}
-1 & -1 \\
-1 & -1
\end{tabular} & -1 & & -1 & & & & 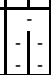 & $1-1$ & & & : & & & & -1 & : & $\left|\begin{array}{cc}3 & 3 \\
2 & 3 \\
-1\end{array}\right|$ & 1 & 1 &. & & 1 & $\begin{array}{l}3 \\
2 \\
2\end{array}$ & 3 & 1 & 4 & 10 & $\mathrm{Ha}$ & & $\mathrm{hg}$ & $\begin{array}{l}\text { Gali veri, Fest-Brom, } \\
\text { Agro int-rep }\end{array}$ \\
\hline \begin{tabular}{|l|} 
Elytrigia repens (L.) \\
Nevski
\end{tabular} & \begin{tabular}{|l|l|}
3 \\
& 2 \\
& 2 \\
\end{tabular} & \begin{tabular}{|c|c|}
- & 1 \\
2 & 3 \\
\end{tabular} & & $-i_{2}^{2}$ & & \begin{tabular}{|l|l|l|} 
& 2 \\
1 & 3 \\
2 & 3 \\
\end{tabular} & $\begin{array}{lll}3 & 3 \\
3 & 3 \\
\end{array}$ & \begin{tabular}{|l|l|}
3 & 3 \\
3 & 3 \\
\end{tabular} & \begin{tabular}{|l}
- \\
2
\end{tabular} & & \begin{tabular}{|l|l|l} 
& 2 \\
3 & 3 \\
\end{tabular} & & & & & \begin{tabular}{c|c|c|} 
& 2 & - \\
3 & 3 & $i$ \\
\end{tabular} & & \begin{tabular}{|l|l|l|}
3 & 1 \\
3 & 3 & 2 \\
3 & 2 \\
\end{tabular} & & \begin{tabular}{|l|l|l} 
& 1 & 1 \\
2 & 1 & 2 \\
3 & 3 & 3
\end{tabular} & \begin{tabular}{|l|l|l|}
2 & 2 & 1 \\
3 & 3 & 3 \\
\end{tabular} & & $\begin{array}{l}52 \\
24 \\
\end{array}$ & & $\begin{array}{l}49 \\
64 \\
\end{array}$ & 25 & 97 & 231 & $A p$ & & $\mathrm{hg}$ & Agro int-rep \\
\hline $\begin{array}{l}\text { Epilobium tetragonum } \\
\text { L. }\end{array}$ & -1. & -1 & & -1 & & & & $-1:-1$ & $\mid-1$ & & & & & & & 1. & $1:-1$ & & & -11 & $1:$ & : & i & -1 & $i$ & 1 & 1 & 1 & $A p$ & & $\mathrm{ch}$ & Stel medi \\
\hline Ephedra distachya L. & $i$ & -1 & & -1 & & & & $-i$ & $\frac{1}{2}$ & & & & & & & 1 & & $i$ & & & & & : & 2 & $:$ & 1 & 2 & 4 & \begin{tabular}{|c|}
$N s$ \\
$\left({ }^{* * * * x)}\right.$ \\
\end{tabular} & & $\mathrm{nf}$ & Fest-Brom, Ammoph \\
\hline Eragrostis minor Host & 0.1 & -1. & & & & & & & & & & & & & & 1 & . & -1 & & & & & & 2 & $i$ & 3 & 4 & 4 & Кe & $\begin{array}{l}\text { Meditrr- } \\
\text { subMediterr }\end{array}$ & $t$ & Stel medi, [Fest vagi] \\
\hline $\begin{array}{l}\begin{array}{l}\text { Eremogone longifolia } \\
\text { (M.Bieb.) Fenzl }\end{array} \\
\end{array}$ & $\begin{array}{ccc}-1 & : & -1 \\
\end{array}$ & $\therefore$ & & 1 & -1 & -1. & $:-1$ & $\because:$ & -1 & & & 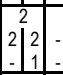 & $1: 1$ & - & $1:-1$ & : & $\therefore 1$ & $: 1:$ & -1 & -1 &.- & & $\begin{array}{l}3 \\
2 \\
\end{array}$ & & $\begin{array}{l}5 \\
2 \\
\end{array}$ & 3 & 10 & 16 & Ns & & $\mathrm{h}$ & Fest-Brom \\
\hline $\begin{array}{l}\text { Eremogone micrade- } \\
\text { nia (P.Smirn.) lkonn. }\end{array}$ & 1 & - & & r & & & & -1. & & & & 4 & & & & . & & i & & $2]^{3}$ & $\begin{array}{ll}2 & 2 \\
1 & 2\end{array}$ & & $\begin{array}{r}4 \\
3 \\
\end{array}$ & $\begin{array}{l}6 \\
6 \\
\end{array}$ & $\begin{array}{c}10 \\
4\end{array}$ & 5 & 18 & 34 & \begin{tabular}{|c|}
$N s$ \\
$(* * * *)$
\end{tabular} & & $\mathrm{h}$ & Fest-Brom \\
\hline \begin{tabular}{|l|}
$\begin{array}{l}\text { Eremogone rigida } \\
\text { (M.Bieb.) Fenzl }\end{array}$ \\
\end{tabular} & $\begin{array}{lll}2 & 2 \\
2 & 2 \\
-\end{array}$ & $-1:$ & & -1 & & & & -1 & & & & 1 & 1 & & -1 & 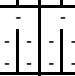 & L & -12 & & & & $\begin{array}{l}1 \\
1\end{array}$ & $\begin{array}{l}2 \\
1 \\
\end{array}$ & $\begin{array}{ll}2 \\
2\end{array}$ & $\begin{array}{l}4 \\
1 \\
\end{array}$ & 2 & 6 & 10 & $\begin{array}{l}N \\
(*) \\
\end{array}$ & & $\mathrm{h}$ & Fest-Brom \\
\hline $\begin{array}{l}\text { Erigeron podolicus } \\
\text { Besser }\end{array}$ & $\mid 1$ & -1 & & & & & & $\because-1$ & & & & -1 & & & & $i$ & . & -1 & & & -1 & 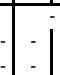 & $i_{2} \quad r$ & & $\dot{2}$ & 2 & 2 & 2 & Ns & & $\mathrm{h}$ & Fest-Brom \\
\hline $\begin{array}{l}\text { Erodium ruthenicum } \\
\text { M.Bieb. } \\
\end{array}$ & $i$ & $i$ & & & & & -1 & $: 1$ & -1 & & & 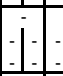 & & 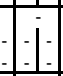 & 1 & 1 & 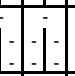 & $i$ & & . & & $: 1$ & 1 & & $i$ & 1 & 1 & 1 & Ns & & $\mathrm{h}$ & Fest-Brom, Gali veri \\
\hline $\begin{array}{l}\begin{array}{l}\text { Erophila verna (L.) } \\
\text { Besser }\end{array} \\
\end{array}$ & 1 & -1 & & 2 & & & & $: 1:-$ &. & & & -1 & & -1 & -1 & 2 & & -1 & 2 & -12 & & 5 & 2 & 10 & $\begin{array}{l}4 \\
\end{array}$ & 7 & 11 & 20 & $\mathrm{Ha}$ & & $t$ & $\begin{array}{l}\text { Fest-Brom, rest vagl, } \\
\text { Poly-Arte, Stel medi, } \\
\text { Ther-Brac }\end{array}$ \\
\hline \begin{tabular}{|l} 
Eryngium campestre \\
L. \\
\end{tabular} & \begin{tabular}{|l|l|l|}
3 & 2 & - \\
2 & 3 & -1 \\
\end{tabular} & -1 & & & & & & -1. & & & & \begin{tabular}{|l|l|l|}
2 & 1
\end{tabular} & -1 & 2 & & 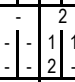 & $\begin{array}{lll}1 & - \\
- & -1 & 1 \\
\end{array}$ & -1 & & & & $\begin{array}{l}2 \\
4 \\
\end{array}$ & & & \begin{tabular}{|l|}
4 \\
6 \\
\end{tabular} & 5 & 16 & 26 & $\mathrm{Ha}$ & & $\mathrm{h}$ & Fest-Brom, Poly-Arte \\
\hline Eryngium planum $\mathrm{L}$. & -1 & $\therefore$ & & & -1 & & $\mid-1$. & $\because:-1$ & $1-1$ & & & 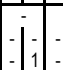 & & -1 & . & $i$ & $\therefore$ & $\therefore$ & & & -1 & & & & $i$ & 1 & 1 & 1 & Ns & & $\mathrm{h}$ & $\begin{array}{l}\begin{array}{l}\text { Gali veri, Moli-Arrh, Fest- } \\
\text { Brom }\end{array} \\
\end{array}$ \\
\hline $\begin{array}{l}\text { Erysimum diffusum } \\
\text { Ehrh. }\end{array}$ & $-1:-1$ & -1 & & & & & & $|1|-\mid$ & & & & $1-1-1$ & 10 & & $\begin{array}{l}1 \\
-1\end{array}|-|-1$ & $\begin{array}{lllll}- & 1 & 1 & 1 \\
\end{array}$ & $1:-1$ & 1 & -1.1 & & $1-1$. & $\mid \begin{array}{l}4 \\
4\end{array}$ & $\begin{array}{l}2 \\
-\end{array}$ & & 2 & 7 & 13 & 18 & $\mathrm{Ha}$ & & $\mathrm{h}$ & Fest-Brom, Poly-Arte \\
\hline
\end{tabular}




\begin{tabular}{|c|c|c|c|c|c|c|c|c|c|c|c|c|c|c|c|c|c|c|c|c|c|c|c|c|c|c|c|c|c|c|c|c|c|c|c|}
\hline & 1 & & 3 & 4 & 5 & 6 & & 8 & 9 & 10 & 11 & 12 & 13 & 14 & 15 & 16 & 17 & 18 & 19 & 20 & \begin{tabular}{|l|l|}
21 & 2 \\
\end{tabular} & & \begin{tabular}{|l|l}
23 & 2 \\
\end{tabular} & 24 & 25 & 26 & & 27 & 28 & 29 & 30 & 31 & 32 & 33 & 34 \\
\hline $\begin{array}{l}\text { Erysimum repandum } \\
\text { L. }\end{array}$ & 1 & & & & & & & & & & & & & & & & & & 1 & & & & & & -1. & $\because j$ & & & 1 & 1 & 1 & Ar & Iranian-Turanian & t & Stel medi \\
\hline $\begin{array}{l}\text { Euonymus europaea } \\
\text { L. }\end{array}$ & & & & & & & & & & & & & & & & & & & 1 & & & & 1. & & -1. & $\because 2$ & & $|\dot{2}|$ & 2 & 2 & 2 & Ns & & nf & Rham-Prun, Quer-Fage \\
\hline $\begin{array}{l}\text { Euphorbia agraria } \\
\text { M.Bieb. }\end{array}$ & - & & & & & & & & 11 & & & & & & & & & & 1 & & & & & & & \begin{tabular}{l|l} 
& 12 \\
2 & 2 \\
4 & 2
\end{tabular} & & $\left|\begin{array}{l}4 \\
2\end{array}\right|$ & 5 & 13 & 17 & $\mathrm{Ha}$ & & $\mathrm{h}$ & Fest-Brom, Agro int-rep \\
\hline \begin{tabular}{l|} 
Euphorbia cyparissias \\
L.
\end{tabular} & 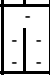 & & & & & & & & & & & & & & & & & & $a_{2}^{2} 2_{2}^{2}$ & & & & & & & \begin{tabular}{l|l}
2 & 2 \\
1 & 1 \\
\end{tabular} & & $\begin{array}{l}3 \\
2 \\
\end{array}$ & 3 & 7 & 12 & $\mathrm{Ha}$ & & $\mathrm{gh}$ & \begin{tabular}{|l|}
$\begin{array}{l}\text { Sedo-Scle, Artemi, Fest- } \\
\text { Brom }\end{array}$ \\
\end{tabular} \\
\hline \begin{tabular}{|l|}
$\begin{array}{l}\text { Euphorbia } \\
\text { kaleniczenkoi Czern. }\end{array}$ \\
\end{tabular} & $i$ & & 1 & & & & & & & & & & & & & & & & 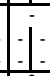 & & & & $i$ & 2 & & \begin{tabular}{l|l}
1 & 1 \\
2 & 2 \\
\end{tabular} & & \begin{tabular}{|l|}
2 \\
3 \\
\end{tabular} & 2 & 7 & 12 & $\begin{array}{c}\mathrm{Ha} \\
\left({ }^{* * * *}\right) \\
\end{array}$ & & $\mathrm{h}$ & Fest-Brom, Artemi \\
\hline $\begin{array}{l}\text { Euphorbia leptocaula } \\
\text { Boiss. }\end{array}$ & -1 & & & & & & & & & & & & & & & & & & $|-|_{1}^{2}$ & & & & & & . & \begin{tabular}{l|l}
1 & 2 \\
2 & 2
\end{tabular} & & $\left|\begin{array}{l}4 \\
2\end{array}\right|$ & 4 & 8 & 13 & Ns & & $\mathrm{h}$ & Fest-Brom \\
\hline $\begin{array}{l}\begin{array}{l}\text { Euphorbia } \\
\text { seguieriana Neck. }\end{array} \\
\end{array}$ & \begin{tabular}{|l|l|} 
& 1 \\
2 & 1 \\
2 & 1 \\
2
\end{tabular} & & & & & & & & & & & & & & & & & & & & & & & & & \begin{tabular}{l|l} 
& 2 \\
3 & 6 \\
3 & 3 \\
\end{tabular} & & $\begin{array}{l}7 \\
3\end{array}$ & 8 & 17 & 23 & Ns & & $\mathrm{h}$ & $\begin{array}{l}\text { Fest vagi, Fest-Brom, } \\
\text { Poly-Arte }\end{array}$ \\
\hline $\begin{array}{l}\text { Euphorbia stepposa } \\
\text { Zoz ex Prokh. }\end{array}$ & $\left|\begin{array}{l|l}2 & 2 \\
3 & 2\end{array}\right|$ & & & & & & & & $23^{3}$ & & & & & & & & & & & & & & & & & \begin{tabular}{l|l}
4 & 10 \\
9 & 10
\end{tabular} & & $\mid \begin{array}{l}18 \\
14\end{array}$ & 11 & 40 & 75 & Ns & & $\mathrm{h}$ & Fest-Brom \\
\hline $\begin{array}{l}\text { Euphorbia subtilis } \\
\text { Prokh. }\end{array}$ & $i$ & & $\therefore$ & & & 21 & & 1 & & & & $|i|^{2}$ & & 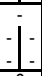 & & & -1 & & 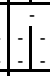 & & -1 & & -1 & & & \begin{tabular}{l|l}
3 & 5 \\
2 & 1 \\
\end{tabular} & & \begin{tabular}{|l}
8 \\
1 \\
\end{tabular} & 7 & 12 & 17 & \begin{tabular}{|c|}
$N s$ \\
$\left({ }^{* * \star *}\right)$ \\
\end{tabular} & & $\mathrm{h}$ & Fest-Brom \\
\hline $\begin{array}{l}\text { Euphorbia virgata } \\
\text { Waldst. \& Kit. }\end{array}$ & \begin{tabular}{|l|l|} 
& 1 \\
2 & - \\
2 & 2 \\
\end{tabular} & & & & & & & 2 & $-i^{2} \quad-\quad-x$ & & & D & & 3 & & & 2 & & $\begin{array}{lll}2 & 2 \\
2 & 2 \\
2\end{array}$ & & & & & & & \begin{tabular}{l|l}
13 \\
19 \\
22 & 18 \\
22
\end{tabular} & & \begin{tabular}{|}
32 \\
41 \\
\end{tabular} & 23 & 93 & 170 & $\mathrm{Ha}$ & & $\mathrm{h}$ & Gali veri, Agro int-rep \\
\hline \begin{tabular}{|l} 
Fagopyrum \\
esculentum Moench
\end{tabular} & $\therefore:$ & & - & & & & & & & & & & ij & & & & & & $i$ & & & & & & & $i i_{2}$ & & $I_{2}$ & 2 & 3 & 3 & $E g$ & C-Asiatic & $t$ & Stel medi \\
\hline \begin{tabular}{|l|} 
Falcaria vulgaris \\
Bernh. \\
\end{tabular} & 2. & & \begin{tabular}{|l|l|}
-1 & 2 \\
2 & 3 \\
\end{tabular} & & & & 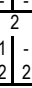 & & 11 & & & 4 & 2 & $\begin{array}{l}2 \\
3 \\
\end{array}$ & & $\begin{array}{l}x_{3}^{2} \\
2 \\
\end{array}$ & 2 & $\mid$\begin{tabular}{l|l|l|}
2 & 2 \\
3 & 3 \\
3
\end{tabular} & $\begin{array}{lll}1 & 2 \\
2 & 2 \\
\end{array}$ & & & & \begin{tabular}{|l|l|}
-1 & 2 \\
2 & 2 \\
\end{tabular} & \begin{tabular}{l|l}
1 & 2 \\
2 &
\end{tabular} & 2 & \begin{tabular}{l|l}
16 & 17 \\
21 & 19 \\
\end{tabular} & & $\mid \begin{array}{l}28 \\
36 \\
\end{array}$ & 22 & 80 & 150 & $\mathrm{Ha}$ & & $\mathrm{h}$ & \begin{tabular}{|l} 
Fest-Brom, Fest vagi, Gali \\
veri, Agro int-rep
\end{tabular} \\
\hline $\begin{array}{l}\text { Fallopia convolvulus } \\
\text { (L.) A.Löve }\end{array}$ & $i$ & & {$\left[\begin{array}{c}1 \\
1 \\
1 \\
1\end{array} \mid\right.$} & & & & & & & & & & & & & & & & $-1_{2}^{1}$ & & & & 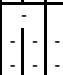 & & . & \begin{tabular}{l|l}
1 & 6 \\
5 & 12
\end{tabular} & & $\mid \begin{array}{c}6 \\
13\end{array}$ & 14 & 25 & 26 & Ar & Asiatic & $\mathrm{t}$ & Stel medi, Robini \\
\hline $\begin{array}{l}\text { Fallopia dumetorum } \\
\text { (L.) Holub }\end{array}$ & $i$ & & $1:$ & & -1 & & & 1 & 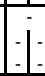 & -1 & & & 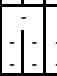 & & & & -1 & & 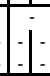 & & 1 & & -1 & 1 & it & $: i_{1}$ & & $|i|$ & 1 & 1 & 1 & $A p$ & & $\mathrm{t}$ & $\begin{array}{l}\text { Gali-Urti, Robin, Rham- } \\
\text { Prun, }\end{array}$ \\
\hline $\begin{array}{l}\text { Festuca pratensis } \\
\text { Huds. }\end{array}$ & -1 & & - & & $1] 2$ & & & -1 & 1-1 & -1 & & & 1 & -1 & & & 1 & & $j$ & & & & $\therefore$ & -1 & -1 & $i i_{3}$ & & $\dot{5}$ & 3 & 4 & 6 & $\mathrm{Ha}$ & & hg & Moli-Arrh, Agro int-rep \\
\hline $\begin{array}{l}\text { Festuca regeliana } \\
\text { Pawl. } \\
\end{array}$ & $\therefore$ & & $\because$ & & & & & -1 & & -1 & & & -1 & & & & & & 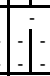 & & & & -1 & & -1 & $: i_{1}$ & & 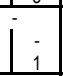 & 1 & 1 & 1 & Ns & & $\mathrm{h}$ & Aste trip, Alth offi \\
\hline $\begin{array}{l}\text { Festuca rupicola } \\
\text { Heuff. } \\
\end{array}$ & i- & & -1 & & & & & & & & & & & & & & & & $-1-$ & & & & -12 & & & \begin{tabular}{l|l}
-3 & 5 \\
4 & 5 \\
\end{tabular} & & \begin{tabular}{|l|}
$\dot{9}$ \\
\end{tabular} & 8 & 9 & 15 & Ns & & $\mathrm{h}$ & Fest-Brom, Gali veri \\
\hline \begin{tabular}{|l|} 
Festuca valesiaca \\
Gaudin \\
\end{tabular} & \begin{tabular}{|l|l|} 
& 2 \\
3 & 3 \\
3 & 2 \\
\end{tabular} & & \begin{tabular}{|l|l|l|}
2 & 2 \\
2 & 2 \\
2 & 1 \\
\end{tabular} & & 01 & & & \begin{tabular}{rl|l}
1 & 2 \\
2 & 2 \\
2 & 2 \\
2
\end{tabular} & $\left|\begin{array}{ll}2 \\
2\end{array}\right|^{2}$ & \begin{tabular}{|l|l|} 
& 2 \\
3 & 2 \\
2 & 1 \\
\end{tabular} & $\frac{1}{2}$ & & \begin{tabular}{|c|c|c|}
3 \\
313
\end{tabular} & & & 2 & 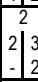 & & $2^{2}$ & & & & \begin{tabular}{|l|l|l|}
2 & \\
2 & 2 & 2 \\
1 & 1 & 2 \\
\end{tabular} & \begin{tabular}{c|c|c|c|c|c|}
2 & 3
\end{tabular} & \begin{tabular}{|l|l|l|}
3 & 3 \\
3 & 3 \\
2 & 2 \\
\end{tabular} & \begin{tabular}{l|l}
17 \\
22 & 20 \\
13 & 13 \\
\end{tabular} & & \begin{tabular}{|l|}
43 \\
22 \\
\end{tabular} & 22 & 85 & 178 & Ns & & $\mathrm{h}$ & $\begin{array}{l}\text { Fest-Brom, Poly-Arte, } \\
\text { Gali veri }\end{array}$ \\
\hline 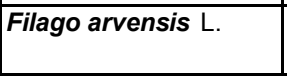 & $\therefore$ & & -1 & & & & & & & $i$ & & & 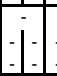 & & & & & & 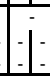 & & -1 & & $i$ & & -1. & $: i_{1}$ & & $i$ & 1 & 1 & 1 & $\mathrm{Ha}$ & & $t$ & \begin{tabular}{|l|}
$\begin{array}{l}\text { Fest-Brom, Fest vagi, } \\
\text { Poly-Arte }\end{array}$ \\
\end{tabular} \\
\hline $\begin{array}{l}\text { Filipendula vulgaris } \\
\text { Moench }\end{array}$ & $i_{i}^{-}$ & & -2 & $1-1-$ & -1 & -1 & . & -1. & 1-1- & $1-$ & -1. & 1 & $-I_{2} \quad$ r & $-1-$ & & & & & $j$ & & 1 & & -1 & & -1 & \begin{tabular}{l|l}
$i$ & 2 \\
$i$ & 2
\end{tabular} & & $\mid \begin{array}{l}3 \\
4\end{array}$ & 3 & 5 & 8 & Ns & & $\mathrm{hg}$ & Moli-Arrh, Gali veri \\
\hline \begin{tabular}{|l|} 
Fragaria viridis \\
Duchesne \\
\end{tabular} & -13 & 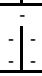 & -1 & 1 & d & & & -1 & .1 & -1 & 1 & 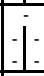 & -1 & .1 & & & & & $\mid$\begin{tabular}{l|l}
2 & 2 \\
3 & 3 \\
\end{tabular} & -12 & 1 & & -1 & -1 & -1 & \begin{tabular}{l|l}
2 & 4 \\
2 & 5 \\
\end{tabular} & & $\mid \begin{array}{c}7 \\
12 \\
\end{array}$ & 6 & 14 & 27 & Ns & & $\mathrm{hc}$ & \begin{tabular}{|l|}
$\begin{array}{l}\text { Gali veri, Trif-Gera, Fest- } \\
\text { Brom }\end{array}$ \\
\end{tabular} \\
\hline Fraxinus excelsior $L$. & -1 & & -1 & & & & & $1-1$ & & I & & & -1. & & & & & & j & & -1 & & -1 & & -1 & $-\mid \begin{array}{l}1 \\
-\end{array}$ & & $\left|\begin{array}{l}1 \\
5\end{array}\right|$ & 4 & 5 & 6 & Ns & & $\mathrm{mf}$ & Sali purp, Robin \\
\hline $\begin{array}{l}\text { Fraxinus pennsylva- } \\
\text { nica Marschall }\end{array}$ & $\therefore:$ & $\because$ & $i$ & \begin{tabular}{|l|l|l} 
& 1 \\
2
\end{tabular} & & & & 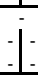 & & i & -1 & & 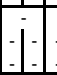 & & 1] & & & & 1 & $\left.\mid \begin{array}{lll}-1 & 1 \\
2\end{array}\right]=$ & -1 & & 1 & & 1. & \begin{tabular}{l|l} 
& 2 \\
3 & 5 \\
\end{tabular} & & \begin{tabular}{|l|}
2 \\
7
\end{tabular} & 5 & 11 & 15 & Ke & North American & $\mathrm{mf}$ & Robin \\
\hline
\end{tabular}




\begin{tabular}{|c|c|c|c|c|c|c|c|c|c|c|c|c|c|c|c|c|c|c|c|c|c|c|c|c|c|c|c|c|c|c|c|c|}
\hline & 1 & 2 & 3 & 4 & 5 & $\frac{6}{6}$ & 7 & 8 & 9 & \begin{tabular}{l|l|}
10 & 11 \\
\end{tabular} & 12 & 1351 & 14 & \begin{tabular}{l|l|}
5 & 16 \\
\end{tabular} & 17 & 18 & 192 & \begin{tabular}{l|l}
20 & 21 \\
\end{tabular} & 1222 & $\frac{2}{1}$ & \begin{tabular}{|l|l|}
3 & 24 \\
\end{tabular} & 25 & $\frac{26}{2}$ & & $\frac{21}{2}$ & 28 & 29 & 30 & 31 & 32 & 33 & 34 \\
\hline $\begin{array}{l}\text { Fumaria schleicheri } \\
\text { Soy.-Willem. }\end{array}$ & $\because:$ & & & & & & & & & & & 17 & & & & & & & & & & i: & \begin{tabular}{|l|l|}
4 & 2 \\
5 & 2
\end{tabular} & $\left|\begin{array}{l}4 \\
5\end{array}\right|$ & $\left|\begin{array}{l}4 \\
3\end{array}\right|$ & 10 & 15 & 18 & Ar & Iranian-Turanian & t & Stel medi \\
\hline $\begin{array}{l}\text { Fumaria vaillantii } \\
\text { Loisel. }\end{array}$ & -1 & & & i1:- & : 1 & i & -1 & & & -1 & -1 & 10 & & & & & & & & & & 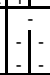 & \begin{tabular}{|l|l|l|}
2 & 1 \\
2 & 1 \\
5 & 3 \\
\end{tabular} & $\begin{array}{l}3 \\
6 \\
6\end{array}$ & $\mid \begin{array}{l}1 \\
4\end{array}$ & 9 & 12 & 15 & Ar & Iranian-Turanian & $\mathrm{t}$ & Stel medi \\
\hline $\begin{array}{l}\text { Gagea erubescens (Bes- } \\
\text { ser) Schult. \& Schult.ff. }\end{array}$ & -1. & & & & & & & & & & & & & & & & & & & & & $i$ & $-i_{3}^{2}$ & & $\mid \begin{array}{l}2 \\
4\end{array}$ & 4 & 5 & 6 & Ns & & g & Fest-Brom, Gali veri \\
\hline $\begin{array}{l}\text { Gagea paczoskii } \\
\text { (Zapał.) Grossh }\end{array}$ & -1. & 2 & & & 工 & & & & & 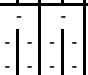 & & & & & & & 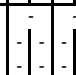 & & & & & $\because$ & 1 & 2. & & 1 & 1 & 2 & Ns & & $\mathrm{g}$ & Fest-Brom, Gali veri \\
\hline $\begin{array}{l}\text { Gagea pusilla (F.W.Sch- } \\
\text { midt) Schult. \& Schult.f. }\end{array}$ & -1 & & i. & & & & & & & $i$ & & & & & & & - & & & & & $i$ & \begin{tabular}{|l|l}
3 & 3 \\
1 & -
\end{tabular} & & $\begin{array}{l}4 \\
-\end{array}$ & 6 & 7 & 9 & Ns & & g & Fest-Brom, Poly-Arte \\
\hline $\begin{array}{l}\text { Galatella villosa (L.) } \\
\text { Rchb.f. }\end{array}$ & -1. & & & & 1 & & & & & 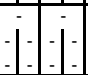 & & & & & & & & & & & & -1 & \begin{tabular}{|l|l|}
1 & 1 \\
1 & 1 \\
\end{tabular} & & \begin{tabular}{|l}
1 \\
1
\end{tabular} & 1 & 4 & 6 & \begin{tabular}{|c|}
$N s$ \\
$(* * * *)$ \\
\end{tabular} & & h & Fest-Brom \\
\hline Galium aparine L. & -1 & & & & & & & & & & & & & & & & & & & & & 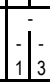 & \begin{tabular}{|l|l|l|}
\multicolumn{1}{|c}{} & \\
1 & 6 \\
8 & 13 \\
\end{tabular} & & \begin{tabular}{|l|}
8 \\
12 \\
26
\end{tabular} & 16 & 37 & 62 & $A p$ & & $\mathrm{t}$ & Gali-Urti, Robin \\
\hline $\begin{array}{l}\begin{array}{l}\text { Galium octonarium } \\
\text { (Klokov) Soó }\end{array} \\
\end{array}$ & -1 & & -1 & & - & & $i$ & & & 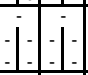 & $1-$ & $\left.\begin{array}{l}1 \\
1\end{array}\right|^{2}$ & & & & & & & & & & $i$ & \begin{tabular}{|l|l|}
2 & 3 \\
1 & 3 \\
\end{tabular} & $\begin{array}{l}2 \\
1 \\
\end{array}$ & $\mid \begin{array}{l}4 \\
-\end{array}$ & 3 & 6 & 7 & Ns & & $\mathrm{h}$ & Fest-Brom \\
\hline $\begin{array}{l}\text { Galium ruthenicum } \\
\text { Willd. }\end{array}$ & \begin{tabular}{|l|l|} 
& -1 \\
2 & 2 \\
\end{tabular} & & $\mid \begin{array}{ll}- \\
2\end{array}$ & & & & & & & 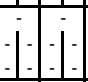 & & 诖: & & & & & & & & & & -1 & \begin{tabular}{|l|l|l|}
\multicolumn{2}{|c|}{} & 2 \\
2 & 2 \\
5 & 4 \\
\end{tabular} & $\begin{array}{l}3 \\
9 \\
\end{array}$ & \begin{tabular}{|l}
4 \\
8 \\
\end{tabular} & 5 & 15 & 27 & Ns & & $\mathrm{h}$ & Fest-Brom, Gali veri \\
\hline Galium spurium $\mathrm{L}$. & $i$ & & & & & & & & & 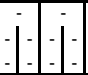 & - & & & & & & & & & & & $i$. & 1 & & 1 & 1 & 1 & 1 & Ar & \begin{tabular}{|l|}
$\begin{array}{l}\text { Mediterr-Iranian- } \\
\text { Turanian }\end{array}$ \\
\end{tabular} & $t$ & Stel medi \\
\hline Galium verum $\mathrm{L}$. & \begin{tabular}{|l|l|} 
& -1 \\
2 & 1 \\
2 & 3 \\
\end{tabular} & & & & & & & & & 1 & & & & & & & & & & & & \begin{tabular}{l|l} 
& 2 \\
3 & 2 \\
\end{tabular} & \begin{tabular}{|l|l|}
9 & 8 \\
8 & 7 \\
\end{tabular} & & $\begin{array}{l}13 \\
15 \\
\end{array}$ & 11 & 35 & 66 & Ns & & $\mathrm{h}$ & Gali veri \\
\hline Geranium pusillum L. & $i$ & & . & & -5 & & $i$ & & & $i$ & & 1 & & & & & & & & & & $i$ & \begin{tabular}{l|l} 
& 1 \\
$i$ & 1 \\
\end{tabular} & & $\mid \begin{array}{l}2 \\
2 \\
\end{array}$ & 1 & 3 & 5 & Ar & Iranian-Turanian & $t$ & Stel medi \\
\hline \begin{tabular}{|l}
$\begin{array}{l}\text { Geranium } \\
\text { robertianum L. }\end{array}$ \\
\end{tabular} & 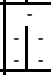 & - & $:=$ & & & & 1 & - & & 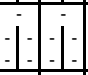 & 站 & : & & & & - & & - - & & 1 & & 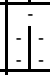 & \begin{tabular}{l|l}
- & 1 \\
- & 1 \\
\end{tabular} & & $\mid \begin{array}{l}1 \\
2 \\
\end{array}$ & 2 & 2 & 3 & $\mathrm{Ha}$ & & ht & Quer-Fage, Stel medi \\
\hline Geum urbanum $\mathrm{L}$. & -1 & & & & & & & & & & & 1. & & & & & & & & & & -1. & \begin{tabular}{|l|l|l|l|} 
& 1 \\
1 & 9 \\
1 & 9 \\
\end{tabular} & & $i_{16}$ & 9 & 11 & 18 & $\mathrm{Ha}$ & & $\mathrm{h}$ & Quer-Fage, Robin \\
\hline $\begin{array}{l}\text { Glaucium cornicu- } \\
\text { latum (L.) J.Rudolph }\end{array}$ & $\therefore$ & & & & & & & & & -1 & & & & & & & & & & & & -1 & \begin{tabular}{|l|l}
2 & - \\
4 & 4 \\
\end{tabular} & $\begin{array}{l}2 \\
6 \\
\end{array}$ & $\mid \begin{array}{l}- \\
4\end{array}$ & 5 & 10 & 12 & $A p$ & & $t$ & Fest-Brom, Stel medi \\
\hline $\begin{array}{l}\text { Glechoma hederacea } \\
\text { L. }\end{array}$ & $\because:$ & -1 & 1 & $i$ & 1 & & $1:$ & & & 1 & & 1 & 1 & & & -1 & $\therefore-1$ & is & 212 & 1 & & $\therefore$ & \begin{tabular}{|l|l} 
& 2 \\
\end{tabular} & $\dot{2}$ & $\mid \dot{4}$ & 2 & 3 & 6 & $\mathrm{Ha}$ & & hc & $\begin{array}{l}\text { Glecho, Moli-Arrh, Sali } \\
\text { purp }\end{array}$ \\
\hline $\begin{array}{l}\text { Goniolimon tataricum } \\
\text { (L.) Boiss. }\end{array}$ & \begin{tabular}{l|l}
2 & $:$ \\
1 & 1 \\
\end{tabular} & & 1 & & . & & $-1-$ & & & 1 & & : & 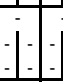 & & & -1. & 1 & 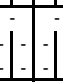 & & & & -1 & \begin{tabular}{|l|l}
1 & - \\
1 & 1 \\
\end{tabular} & $\begin{array}{l}2 \\
1 \\
\end{array}$ & $i$ & 1 & 3 & 4 & \begin{tabular}{|c|}
$N s$ \\
$(* * *)$ \\
\end{tabular} & & $\mathrm{h}$ & Fest-Brom \\
\hline $\begin{array}{l}\text { Gypsophila paniculata } \\
\text { L. } \\
\end{array}$ & \begin{tabular}{|l|l|} 
& 1 \\
2 & 2 \\
2 & 1 \\
\end{tabular} & & \begin{tabular}{|l|l|l|l|l|l|l|l|}
2 & 2 & 2 \\
3 & 2 & -
\end{tabular} & & & & \begin{tabular}{|l|l|} 
& 2 \\
2 & 2 \\
3 & 2 \\
\end{tabular} & $t$ & \begin{tabular}{|l|l|} 
& 1 \\
2 & 2 \\
3 & 3 \\
\end{tabular} & $\begin{array}{ll}-1 \\
2 & 1 \\
\end{array}$ & i. & -1 & 2 & & i & & $\left.\begin{array}{ll}1 & \\
1 \\
1\end{array}\right]$ & \begin{tabular}{l|l}
-5 & 1 \\
& 1
\end{tabular} & & & & $i$ & \begin{tabular}{|l|l|}
9 & 9 \\
12 & 13 \\
18 & 15 \\
\end{tabular} & \begin{tabular}{|l|l|}
3 & 22 \\
& 40 \\
\end{tabular} & \begin{tabular}{|l|}
20 \\
28 \\
28
\end{tabular} & 20 & 67 & 122 & Ns & & $\mathrm{h}$ & Fest vagi, Sedo-Scle \\
\hline $\begin{array}{l}\text { Helictotrichon pubes- } \\
\text { cens (Huds.) Pilg. }\end{array}$ & -1. & & & & & & & & & . & & $2^{2}$ & & & & & 1 & 1 & & & & $i$ & \begin{tabular}{|l|l}
1 & 1 \\
- & 1.
\end{tabular} & & 2 & 1 & 2 & 4 & Ns & & $\mathrm{t}$ & Fest-Brom \\
\hline Helianthus annuus $\mathrm{L}$. & $1-$ & & & & & & & & & . & & & & & & & & & & & & 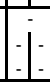 & \begin{tabular}{|l|l}
- & 1 \\
1 &
\end{tabular} & & 1 & 2 & 2 & 2 & $E g$ & North American & $t$ & Stel medi \\
\hline $\begin{array}{l}\begin{array}{l}\text { Helichrysum arena- } \\
\text { rium (L.) Moench }\end{array} \\
\end{array}$ & \begin{tabular}{l|l|}
2 & 2 \\
2 & 1 \\
\end{tabular} & & 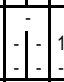 & $1:-1$ & 1] 1 i. & & & & (11) & . & & \begin{tabular}{l|l|l}
1 & 2 & 2 \\
2 & - & 3 \\
\end{tabular} & \begin{tabular}{l|l|l}
2 & 2 & - \\
3 & 2 & - \\
\end{tabular} & $\left.\mid \begin{array}{ll}2 & 2 \\
2 & 2\end{array}\right]$ & $4-$ & \begin{tabular}{|l|l|} 
& 2 \\
2 & 2 \\
\end{tabular} & \begin{tabular}{rr|r|}
1 & -1 \\
-1 & 1 \\
\end{tabular} & 1 i. & & & & 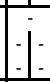 & \begin{tabular}{|l|l|}
8 & 9 \\
8 & 5 \\
\end{tabular} & & \begin{tabular}{|c|}
15 \\
8 \\
\end{tabular} & 13 & 35 & 55 & Ns & & $\mathrm{h}$ & $\begin{array}{l}\text { Fest vagi, Fest-Brom, } \\
\text { Sedo-Scle }\end{array}$ \\
\hline $\begin{array}{l}\text { Heracleum sibiricum } \\
\text { L. }\end{array}$ & $\therefore$ & & - & : & $1-1-1$ & & & & & i. $1:-1$ & $1-$ &. & . & & & & 1 & 1. & & & & $1-1$ & i 1. & & 4 & 3 & 4 & 5 & $\mathrm{Ha}$ & & th & $\begin{array}{l}\text { Moli-Arrh, Sali purp, } \\
\text { Artemi }\end{array}$ \\
\hline $\begin{array}{l}\text { Herniaria besseri } \\
\text { Fisch. ex Hornem. }\end{array}$ & 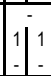 & & $1:-1$ & -1 & -1 & & & & & 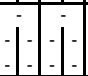 & &. & 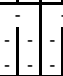 & & & 1 & $\begin{array}{lll}- & -1 \\
& -1 & 1 \\
\end{array}$ & $\begin{array}{lll}-1 & -1 \\
-1 & -1\end{array}$ & & & & $i$ & \begin{tabular}{|l|l}
1 & 1 \\
& $!$ \\
\end{tabular} & & \begin{tabular}{|l|}
1 \\
-
\end{tabular} & 1 & 2 & 2 & Ns & & c & Fest-Brom \\
\hline
\end{tabular}




\begin{tabular}{|c|c|c|c|c|c|c|c|c|c|c|c|c|c|c|c|c|c|c|c|c|c|c|c|c|c|c|c|c|c|c|c|c|}
\hline & \begin{tabular}{l|l}
1 & 2 \\
\end{tabular} & \begin{tabular}{|l|l|}
3 & 4 \\
\end{tabular} & 5 & 6 & $I$ & & 59 & \begin{tabular}{l|l}
10 & 11 \\
1
\end{tabular} & 112 & 13 & \begin{tabular}{|l|l|}
14 & 15 \\
\end{tabular} & \begin{tabular}{l|l|}
5 & 16 \\
\end{tabular} & 17 & 18 & \begin{tabular}{|l|l|}
19 & 20 \\
\end{tabular} & \begin{tabular}{l|l|}
0 & 21 \\
\end{tabular} & \begin{tabular}{|l|l|l|l|l|}
22 & 2 \\
\end{tabular} & 23 & 24 & & & & & 7 & 28 & $\underline{2}$ & & 30 & 31 & 32 & 33 & 34 \\
\hline Herniaria glabra L. & & & & & & & & & & & & & & & $2 \mid 1$ & & & & & & & & & 1 & 2 & 3 & & 4 & $\mathrm{Ha}$ & & $t$ & Fest vagi, Stel medi \\
\hline $\begin{array}{l}\text { Herniaria polygama } \\
\text { J.Gay }\end{array}$ & $: \begin{array}{lll}-1 & -1 \\
\end{array}$ & $\therefore \begin{array}{rr}-1 \\
-1\end{array}$ & & & & & & & & & & & & & $:$ & 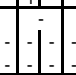 & & & & & 1 & & & & 1 & 2 & & 3 & $\mathrm{Ha}$ & & $t$ & Fest vagi, Stel medi \\
\hline Hesperis tristis $\mathrm{L}$. & & & & & & & & & & & & & & & & & & & & & & ? & & \begin{tabular}{|l|}
1 \\
-
\end{tabular} & 1 & 1 & & 1 & $\begin{array}{c}N s \\
(* * *)\end{array}$ & & th & Fest-Brom \\
\hline $\begin{array}{l}\text { Hieracium echioides } \\
\text { Lumn. }\end{array}$ & \begin{tabular}{|l|l|l|}
1 & 1 & - \\
2 & - & - \\
\end{tabular} & $\therefore: \begin{array}{lll}1 & 1 \\
2 & 1\end{array}$ & \begin{tabular}{|l|l|l|l|}
2 & 2 \\
2 & 1
\end{tabular} & & & & & & & & & & & $\left|\begin{array}{cc|c}-1 & 1 \\
2 & 1\end{array}\right|$ & 1 & & & & & & & $\begin{array}{l}9 \\
8\end{array}$ & & \begin{tabular}{|l|}
12 \\
11
\end{tabular} & 12 & 37 & & 58 & Ns & & $\mathrm{h}$ & $\begin{array}{l}\text { Fest-Brom, Fest vagi, } \\
\text { Sedo-Scle }\end{array}$ \\
\hline Hieracium pilosella $\mathrm{L}$. & $\therefore$. & & & & & & & & & & & & & & & & & & & & & 2 & & & 2 & 2 & & 3 & $\mathrm{Ha}$ & & $\mathrm{h}$ & Fest vagi, Sedo-Scle \\
\hline \begin{tabular}{|l|} 
Hieracium \\
umbellatum $\mathrm{L}$.
\end{tabular} & -1. & & 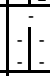 & & & & & & & & $\because$ & & & & & -1. & & & & 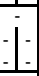 & & $\begin{array}{l}1 \\
1 \\
\end{array}$ & & \begin{tabular}{|l|}
1 \\
1 \\
\end{tabular} & 1 & 3 & & 3 & Ns & & $\mathrm{h}$ & \begin{tabular}{|l} 
Gali veri, Trif-Gera, Quer \\
pube-petr
\end{tabular} \\
\hline $\begin{array}{l}\text { Hieracium virosum } \\
\text { Pall. }\end{array}$ & - & & & & & & & & & & & & & & & & & & & & & $\begin{array}{l}1 \\
2\end{array}$ & & \begin{tabular}{|l|}
2 \\
3
\end{tabular} & 3 & 7 & & 11 & $\begin{array}{c}N s \\
(* * *)\end{array}$ & & $\mathrm{h}$ & Fest-Brom \\
\hline $\begin{array}{l}\begin{array}{l}\text { Hierochloë repens } \\
\text { (Host) P.Beauv. }\end{array} \\
\end{array}$ & $\begin{array}{ccc}-1 & -1 \\
-1 & -1 \\
\end{array}$ & \begin{tabular}{|l|l|l|l|l}
- & 2 \\
-1 & 1 & 3 \\
2 & 2 & 3 & 3 \\
2 & 2
\end{tabular} & & & & & & & & & & & & & & & & & & & & 7 & & $\begin{array}{l}10 \\
15 \\
\end{array}$ & 9 & 23 & & 45 & Ns & & $\mathrm{h}$ & Gali veri \\
\hline \begin{tabular}{|l|}
$\begin{array}{l}\text { Holosteum } \\
\text { umbellatum } \mathrm{L} .\end{array}$ \\
\end{tabular} & -1 & & & & & & & & & & & & & & & -1 & & & & & & 3 & & \begin{tabular}{|l|}
4 \\
4 \\
\end{tabular} & 5 & 11 & & 18 & $\mathrm{Ha}$ & & $\mathrm{t}$ & $\begin{array}{l}\text { Fest-Brom, Fest vagi, } \\
\text { Poly-Arte, Stel medi,Crit- } \\
\text { Limo }\end{array}$ \\
\hline $\begin{array}{l}\begin{array}{l}\text { Hyacinthella leucophaea } \\
\text { (K.Koch) Schur }\end{array} \\
\end{array}$ & $\therefore: 2$ & $\therefore$ & $1:$ & & & & & & & & -1 & & & & 1 & -1 & & & & & & $\begin{array}{l}1 \\
1 \\
1\end{array}$ & 2 & $\begin{array}{l}1 \\
1 \\
\end{array}$ & 3 & 4 & & 5 & $\begin{array}{c}N s \\
(* * * *) \\
\end{array}$ & & $\mathrm{g}$ & Fest-Brom \\
\hline $\begin{array}{l}\text { Hylotelephium } \\
\text { maximum (L.) Holub }\end{array}$ & 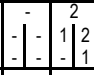 & & : & & & & & & & & & & & & & & & & & & & $\begin{array}{r}2 \\
2 \\
\end{array}$ & & \begin{tabular}{|l}
4 \\
2 \\
\end{tabular} & 2 & 7 & & 10 & Ns & & $\mathrm{gh}$ & $\begin{array}{l}\text { Sedo-Scle, Moll-Arrh, } \\
\text { Gera-Trif, Quer pube- } \\
\text { petr. Robin }\end{array}$ \\
\hline $\begin{array}{l}\text { Hylotelephium polo- } \\
\text { nicum (Blocki) Holub }\end{array}$ & $\because$ & & 1 & & & & & $2 \mid \begin{array}{lll}2 & 2 & 1\end{array}:-$ & & & 1 & & & & 1 & -1 & & & & 1 & & $\begin{array}{l}7 \\
4\end{array}$ & & $\mid \begin{array}{c}13 \\
4\end{array}$ & 9 & 25 & & 38 & Ns & & $\mathrm{h}$ & Fest-Brom \\
\hline Hyoscyamus niger L. & & & 1 & & & & & & & & & [2] & & \begin{tabular}{|l|l|l|} 
& -1 & \\
2 & - & \\
\end{tabular} & & $i$ & & & & $2^{2}$ & & $\begin{array}{l}2 \\
4\end{array}$ & $\begin{array}{c}5 \\
10\end{array}$ & \begin{tabular}{|l|}
2 \\
4 \\
\end{tabular} & 12 & 23 & & 28 & Ar & Iranian-Turanian & th & Stel medi, Artemi \\
\hline \begin{tabular}{|l|} 
Hypericum elegans \\
Stephan ex Willd. \\
\end{tabular} & 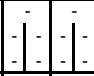 & 1 & $i$ & & & & & & & & \begin{tabular}{|l|l}
2 & 2 \\
2 & 2 \\
\end{tabular} & & & $\left.\begin{array}{ll|l}2 & 2 \\
2 & 2\end{array}\right]$. & & j. & & & & & & $\begin{array}{l}9 \\
8 \\
\end{array}$ & $\begin{array}{l}13 \\
10 \\
\end{array}$ & \begin{tabular}{|l|}
14 \\
15 \\
\end{tabular} & 12 & 35 & & 57 & Ns & & $\mathrm{h}$ & Fest-Brom, Gali veri \\
\hline \begin{tabular}{|l}
$\begin{array}{l}\text { Hypericum } \\
\text { perforatum L. }\end{array}$ \\
\end{tabular} & -1. & $\begin{array}{l}1 \\
-1 \\
2\end{array}$ & \begin{tabular}{|l|l|}
2 & 2 \\
2 & 2 \\
\end{tabular} & & & & & i. & & & 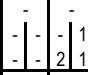 & & & & & $\left.\begin{array}{c}- \\
1\end{array}\right]$ & & & & & & $\begin{array}{l}14 \\
14 \\
\end{array}$ & $\begin{array}{l}15 \\
22 \\
\end{array}$ & \begin{tabular}{|l|}
18 \\
23 \\
\end{tabular} & 18 & 60 & & 86 & $\mathrm{Ha}$ & & $\mathrm{h}$ & Gali veri \\
\hline $\begin{array}{l}\text { Iris hungarica Waldst. } \\
\text { \& Kit. }\end{array}$ & $\therefore:-1:$ & $: 1$ & $1:$ & & & & & $-1:$ & & $-2_{2}^{2}$ & $1:$ & & -1 & $1-1 .-1$ & 1 & -1 & & & & & & $\begin{array}{l}2 \\
2\end{array}$ & - & $\begin{array}{l}5 \\
3 \\
\end{array}$ & 2 & 5 & & 10 & \begin{tabular}{|l|}
$N s$ \\
$(* * *)$
\end{tabular} & & g & Fest-Brom \\
\hline Iris pumila $\mathrm{L}$. & $\therefore:$ & & 1 & & & & & . & & -1 & 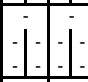 & & & 2 & 2 & -1. & - & & & & & 3 & $\begin{array}{l}10 \\
1 \\
\end{array}$ & 4 & 5 & 13 & & 20 & \begin{tabular}{|c|}
$N s$ \\
$(* * *)$
\end{tabular} & & $\mathrm{g}$ & Fest-Brom \\
\hline Iva xanthiifolia Nutt. & \begin{tabular}{rl|l}
- & 1 \\
1 & - \\
\end{tabular} & & & & & & & & & 11 & 1 & & & & & 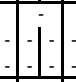 & & & & & & $\begin{array}{l}2 \\
5 \\
\end{array}$ & 3 & \begin{tabular}{|l|}
2 \\
5 \\
\end{tabular} & 10 & 15 & & 15 & Ke & North American & $t$ & Stel medi, Artemi \\
\hline \begin{tabular}{|l|} 
Jurinea calcarea \\
Klokov
\end{tabular} & $\therefore:-1$ & $: 1:$ & $1:$ & & & & & \begin{tabular}{|c|c|c|} 
& -1 \\
& -1 & -1
\end{tabular} & -1 & 1 & $\because-1$ & 1 & -1 & $\therefore$ & $1:$ & $: 1$ & .1 & & & -1 & 1 & 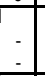 & 1. & - & 1 & 2 & & 2 & \begin{tabular}{|c|}
$N s$ \\
$(* * *)$
\end{tabular} & & $\mathrm{h}$ & Fest-Brom \\
\hline \begin{tabular}{|l} 
Jurinea salicifolia \\
Grun. \\
\end{tabular} & \begin{tabular}{l|l|l|l|l|l|}
2 & 2 & -1 \\
2 & 2 & -1 & -1
\end{tabular} & \begin{tabular}{|l|l|l|l}
2 & 2 & -1 \\
2 & 2 & -1 &
\end{tabular} & $1:$ & & & & & 1 & & 2 & $1:$ & & & -1 & $1:$ & 1 & & & & & & $\begin{array}{l}3 \\
2 \\
\end{array}$ & $\begin{array}{l}6 \\
4 \\
\end{array}$ & \begin{tabular}{|l|}
6 \\
4 \\
\end{tabular} & 3 & 11 & & 22 & $\begin{array}{c}N s \\
(* * * *) \\
\end{array}$ & & $\mathrm{h}$ & Fest-Brom \\
\hline $\begin{array}{l}\text { Knautia arvensis (L.) } \\
\text { Koult. }\end{array}$ & i & 1 & -1 & & & & & -1 & & & 1 & & & & 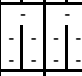 & $i$ & & & & & & 2 & & \begin{tabular}{|l}
2 \\
\end{tabular} & 3 & 3 & & 3 & $\mathrm{Ha}$ & & $\mathrm{h}$ & Moli-Arrh, Trii-Gera \\
\hline $\begin{array}{l}\text { Kochia prostrata (L.) } \\
\text { Schrad. } \\
\end{array}$ & $1:$ & 2 & \begin{tabular}{|l|l|}
2 & 2 \\
-2 & 2 \\
\end{tabular} & & $2 \mid$ & & & 1 & 2 & 3 & 1 & & & & & & & & & & & $\begin{array}{r}11 \\
4 \\
\end{array}$ & & \begin{tabular}{|l|}
14 \\
14 \\
5
\end{tabular} & 15 & 47 & & 69 & Ns & & c & Fest-Brom, Crit-Stat \\
\hline $\begin{array}{l}\text { Koeleria cristata (L.) } \\
\text { Pers. }\end{array}$ & \begin{tabular}{|l|c|c|c|}
2 & 2 \\
2 & 1 & 2 & 2 \\
2 & -1 & 2 & 1 \\
\end{tabular} & $\left|\begin{array}{l|l}\mid & 2\end{array}\right|$ & \begin{tabular}{|c|c|}
2 & 2 \\
2 & 2 \\
1 & 1
\end{tabular} & & $\mid \begin{array}{ccc}2 \\
1\end{array}$ & 2 & & $2 \mid$ & 2! & 2 & 3 & 2 & 2 & -12 & \begin{tabular}{ll|l|l|l}
3 & 3 & 2 & 1 \\
2 & 1 & 2 & -1
\end{tabular} & \begin{tabular}{ll|l|l|l|l|l|l|l}
1 & 3 & 3 \\
2 & 2 & 2 & 2
\end{tabular} & \begin{tabular}{l|l|l}
3 & 3 & 2 \\
2 & -1 &
\end{tabular} & & & & & 23 & $\begin{array}{l}50 \\
33\end{array}$ & \begin{tabular}{|l|}
29 \\
51 \\
26
\end{tabular} & 25 & 10 & & 189 & Ns & & $\mathrm{h}$ & Fest-Brom \\
\hline
\end{tabular}




\begin{tabular}{|c|c|c|c|c|c|c|c|c|c|c|c|c|c|c|c|c|c|c|c|c|c|c|c|c|c|c|c|c|c|c|c|}
\hline & 1 & 2 & 3 & 4 & 5 & 6 & 7 & 8 & 9 & 10 & 11 & \begin{tabular}{|l|l|}
12 & 13 \\
\end{tabular} & 14 & 15 & 16 & \begin{tabular}{|l|l|}
17 & 18 \\
\end{tabular} & 19 & 202 & \begin{tabular}{l|l}
21 & 22 \\
\end{tabular} & \begin{tabular}{|l|l|}
223 \\
\end{tabular} & 24 & 25 & 26 & 27 & 28 & 29 & 30 & 31 & 32 & 33 & 34 \\
\hline Lactuca serriola $\mathrm{L}$. & $i$ & & & & $d$ & & & & & & & \begin{tabular}{|c|c|c|} 
& 2 & - \\
1 & 2 & -1 \\
2 & 2 & 2 \\
\end{tabular} & & & & & $-i i_{1}^{1}$ & $\mid \begin{array}{ll}-1 \\
1\end{array}{ }_{1}^{1}$ & & & $\left|\begin{array}{ll}2 \\
3 \\
3\end{array}\right| \begin{array}{l}2 \\
2\end{array} \mid$ & & \begin{tabular}{c|c|}
9 & \\
6 & 11 \\
17 & 20 \\
\end{tabular} & \begin{tabular}{|l|l|}
\multicolumn{1}{|c|}{14} & 13 \\
29 & 27 \\
\end{tabular} & 21 & 63 & 90 & Ar & \begin{tabular}{|l|}
$\begin{array}{l}\text { Mediter-Iranian- } \\
\text { Turanian }\end{array}$ \\
\end{tabular} & th & Artemi, Stel medi \\
\hline $\begin{array}{l}\text { Lamium amplexicaule } \\
\text { L. }\end{array}$ & -1 & & & & 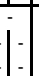 & & & & & & & & & & & & $i$ & & & & & $\left.\mid \begin{array}{l}1 \\
2\end{array}\right]$ & \begin{tabular}{l|l|l}
3 & 4 \\
5 & 2
\end{tabular} & \begin{tabular}{l|l|}
6 & 4 \\
8 & 2 \\
\end{tabular} & 6 & 19 & 25 & Ar & $\begin{array}{l}\text { Mediterr-Iranian- } \\
\text { Turanian }\end{array}$ & $t$ & $\begin{array}{l}\begin{array}{l}\text { Stel medi, [Poly-Arte], } \\
\text { [Fest-Brom] }\end{array} \\
\text { [Fro }\end{array}$ \\
\hline Lamium purpureum $\mathrm{L}$. & & & 1 & & ; & & & & & & & & & & & & 1 & & i & 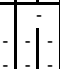 & & 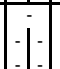 & \begin{tabular}{l|l}
1 & 1 \\
1 &
\end{tabular} & \begin{tabular}{l|l|l}
1 & 1 \\
2 & - &
\end{tabular} & 1 & 3 & 4 & Ar & NE-Mediterr & th & Stel medi, Bident \\
\hline $\begin{array}{l}\text { Lamium paczos- } \\
\text { kianum Worosch. }\end{array}$ & $1:$ & & & & & & & & & & & & & & & & 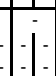 & & & & & $|1|$ & $i \mid:$ & $i j$ & 1 & 1 & 1 & $\mathrm{Ha}$ & & $\mathrm{t}$ & Fest-Brom, Stel medi \\
\hline $\begin{array}{l}\text { Lappula squarrosa } \\
\text { (Retz.) Dumort. }\end{array}$ & -1 & & & & 1 & & & & & & & & & & & & -1 & & & -1 & & \begin{tabular}{|l|l|l|} 
& 1 \\
2 & 2 \\
1 & 1
\end{tabular} & \begin{tabular}{l|l}
6 & 1 \\
4 & 7 \\
10 & 8
\end{tabular} & \begin{tabular}{c|c|}
5 & 8 \\
11 & 8 \\
\end{tabular} & 19 & 35 & 41 & Ar? & $\begin{array}{l}\text { sub-Mediterr-sub- } \\
\text { continent- } \\
\text { Eurasiatic }\end{array}$ & th & \begin{tabular}{|l|} 
Stel medi, Artemi, [Fest- \\
Brom]
\end{tabular} \\
\hline $\begin{array}{l}\text { Lathyrus pannonicus } \\
\text { (Jacq.) Garcke }\end{array}$ & it & & & & 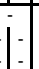 & & & & & & & & & & & & $i$ & 1 & j. & 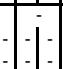 & & $-i$ & -1 & $: 1: 1$ & 1 & 1 & 1 & \begin{tabular}{|c|}
$N s$ \\
$\left({ }^{* * * *}\right)$ \\
\end{tabular} & & $\mathrm{h}$ & Fest-Brom \\
\hline Lathyrus pratensis $\mathrm{L}$. & & & & & 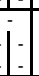 & & & & & & & & & & & & -1 & 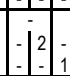 & i) & -1 & -1 & $\because 1$ & $i$ & \begin{tabular}{|l|l|} 
& 2 \\
& 2 \\
\end{tabular} & 2 & 2 & 3 & $\mathrm{Ha}$ & & $\mathrm{hg}$ & Moli-Arrh \\
\hline Lathyrus sylvestris $\mathrm{L}$. & & & & & 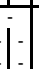 & & & & & & & & & & & & $i$ & & & & & & & & 1 & 1 & 1 & Ns & & $\mathrm{h}$ & Trif-Gera, Quer pube \\
\hline Lathyrus tuberosus $\mathrm{L}$. & - & & & & 1 & & & & & & & & & & 2. & & -1 & & & -1 & & 1 & \begin{tabular}{c|l}
8 & 11 \\
15 & 13 \\
\end{tabular} & \begin{tabular}{|l|l|}
\multicolumn{1}{|c|}{} & 1 \\
13 & 19 \\
30 & 26 \\
\end{tabular} & 16 & 50 & 92 & Ar & Iranian-Turanian & $\mathrm{hg}$ & $\begin{array}{l}\text { Stel medi, Gali-veri, , Agr } \\
\text { int-rep, [Trif-Gera], [Fest- } \\
\text { Brom] }\end{array}$ \\
\hline $\begin{array}{l}\text { Lavatera thuringiaca } \\
\mathrm{L} \text {. } \\
\end{array}$ & in & & & & 1 & & & & & & & & & & 1 & & $-i$ & $1-1$ & 12 & $\mid-1$ & $\mid \begin{array}{l}1 \\
1 \\
1\end{array} 1$ & i. & \begin{tabular}{c|c}
6 & \\
6 & 6 \\
12 & 13 \\
\end{tabular} & 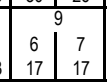 & 18 & 43 & 56 & Ns & & $\mathrm{h}$ & Gali veri, Fest-Brom \\
\hline Leonurus cardiaca L. & -1 & & & & & & & & & & & & & & & & $i$ & & & & & & & \begin{tabular}{|l|l|}
1 & 1 \\
2 & 4 \\
\end{tabular} & 5 & 7 & 8 & Ar & \begin{tabular}{|l|}
$\begin{array}{l}\text { Mediter-Iranian- } \\
\text { Turanian }\end{array}$ \\
\end{tabular} & $\mathrm{h}$ & Artemi \\
\hline \begin{tabular}{|l|} 
Leonurus \\
glaucescens Bunge
\end{tabular} & 1 & & & & 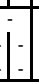 & & & & & & & & & & & & 1 & & i & & & & \begin{tabular}{l|l}
-3 & 6 \\
3 & 6 \\
\end{tabular} & \begin{tabular}{|l|l|}
-1 & - \\
& 7 \\
\end{tabular} & 6 & 9 & 11 & $\mathrm{Ha}$ & & $\mathrm{h}$ & Artemi \\
\hline $\begin{array}{l}\text { Lepidium densiflorum } \\
\text { Schrad. }\end{array}$ & -1. & & & & 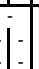 & & & & & & & -1 & & & & & 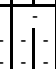 & & j & 1 & $\because 1$ & $-i$ & $i j$ & $i j$ & 2 & 2 & 2 & Ke & North American & $t$ & Stel medi \\
\hline $\begin{array}{l}\text { Libanotis sibirica } \\
\text { C.A.Mey. } \\
\end{array}$ & -1 & \begin{tabular}{|l|}
-2 \\
2 \\
2 \\
2 \\
2
\end{tabular} & & & 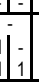 & & & & & & & & & & & & $i$ & 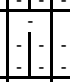 & i & -1 & - & -1 & \begin{tabular}{l|l} 
& 1 \\
2 & 2 \\
3 & 3 \\
\end{tabular} & 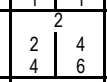 & 3 & 11 & 18 & Ns & & $\mathrm{h}$ & Fest-Brom, Gali veri \\
\hline \begin{tabular}{|l}
$\begin{array}{l}\text { Limonium tomentellum (Boiss.) Kunt-ze } \\
\text { ssp. alutaceum (Stev.) Moysiyenko }\end{array}$ \\
\end{tabular} & $\because i$ & & & & 1 & & & & & & & &.- & & -1 & & 1 & & 1 & 1 & 1 & $\because:$ & $: \quad i$ & $\therefore:{ }_{1}$ & 1 & 1 & 1 & \begin{tabular}{|c|}
$N s$ \\
$(* \star \star *)$ \\
\end{tabular} & & $\mathrm{h}$ & Fest-Brom, Fest-Pucc \\
\hline $\begin{array}{l}\text { Linaria biebersteinii } \\
\text { Besser }\end{array}$ & & & & & $\frac{1}{1}$ & & \begin{tabular}{|l|l|} 
& 2 \\
2 & 2 \\
\end{tabular} & & \begin{tabular}{|l|l|l|}
2 & 2 \\
& 2 \\
\end{tabular} & 2 & & 212 & \begin{tabular}{l|l}
2 & 2 \\
2 & 2 \\
2
\end{tabular} & & \begin{tabular}{|c|c|c|} 
& 2 \\
2 & 2 \\
2 & 2 \\
\end{tabular} & $\left.\right|^{2}$ & $2 .-1$ & & i & & & -1 & \begin{tabular}{c|c}
9 & 10 \\
13 & 10 \\
\end{tabular} & \begin{tabular}{l|l|}
18 & 19 \\
25 & 16 \\
\end{tabular} & 17 & 49 & 89 & $\begin{array}{ll}\mathrm{Ha} \\
(*) \\
\end{array}$ & & $\mathrm{h}$ & \begin{tabular}{|l|}
$\begin{array}{l}\text { Fest-Brom, Gali veri, Agro } \\
\text { int-rep, Moli-Arrh }\end{array}$ \\
\end{tabular} \\
\hline \begin{tabular}{|l|} 
Linaria genistifolia \\
(L.) Mill. \\
\end{tabular} & L & & 1 & & 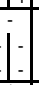 & & & $\begin{array}{lll}2 & 1 & \\
2 & 1 & a\end{array}$ & & & & & & & & & 1 & & 1 & & & & \begin{tabular}{l|l}
1 & 3 \\
1 & 3 \\
\end{tabular} & \begin{tabular}{l|l|}
2 & 3 \\
2 & 3 \\
\end{tabular} & 4 & 8 & 10 & $\mathrm{Ha}$ & & $\mathrm{h}$ & Fest vagi, Fest-Brom \\
\hline Linaria vulgaris Mill. & $\therefore$ & & & & & & & $\left.i_{2}\right]_{2}$ & & $\mid \begin{array}{l}2 \\
2\end{array}$ & 112 & & & $\dot{2} 2$ & & 2 & $2 i$ & & $1]$ & i) & $\begin{array}{lll} & 1 \\
1 & 2 \\
\end{array}$ & \begin{tabular}{|l|l|}
1 & \\
2 & 1 \\
\end{tabular} & \begin{tabular}{c|c}
6 & 8 \\
16 & 17 \\
\end{tabular} & \begin{tabular}{|l|l|}
7 & 4 \\
73 & 10 \\
23 & 26 \\
\end{tabular} & 23 & 51 & 70 & $A p$ & & $\mathrm{~h}$ & Agro int-rep, Moli-Arrh \\
\hline Linum hirsutum $\mathrm{L}$. & $i$ & & . & & 1 & & & & & -1 & & & & & & & i. & & II & -1 & & & $i$ & $-j i$ & 1 & 1 & 1 & \begin{tabular}{|c|}
$N s$ \\
$\left({ }^{* * * *}\right)$ \\
\end{tabular} & & $\mathrm{h}$ & Fest-Brom \\
\hline $\begin{array}{l}\text { Linum usitatissimum } \\
\mathrm{L} .\end{array}$ & 1 & & 1 & & 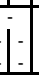 & & & & & -1 & & & & & & 1 & 1 & & -1 & -1 & & -1 & 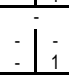 & $: i_{1}$ & 1 & 1 & 1 & $E g$ & unknown & $\mathrm{t}$ & Stel medi \\
\hline Lolium perenne L. & & & 1 & & & & & & & & & & & & & & 1 & & & & & -1 & 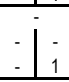 & $: i_{2}$ & 1 & 1 & 2 & $A p$ & & $\mathrm{~h}$ & Plan majo \\
\hline Lotus corniculatus L. & -12 & & & & & & & & & 1 & & & & & $\left|\begin{array}{l}2 \\
2\end{array}\right|$ & i & $1:$ & & & -1 & & & \begin{tabular}{l|l}
1 & 1 \\
1 & 3 \\
\end{tabular} & \begin{tabular}{|l|l|}
2 & - \\
2 & 5 \\
\end{tabular} & 3 & 5 & 9 & $\mathrm{Ha}$ & & $\mathrm{h}$ & Moli-Arrh \\
\hline Lycopsis orientalis $\mathrm{L}$. & & & & & & & & & & & & & & & & & & & & & & & & \begin{tabular}{l|l}
$\vdots$ & - \\
1 &
\end{tabular} & 1 & 1 & 1 & $A p$ & & $\mathrm{t}$ & Stel medi \\
\hline
\end{tabular}




\begin{tabular}{|c|c|c|c|c|c|c|c|c|c|c|c|c|c|c|c|c|c|c|c|c|c|c|c|c|c|c|c|c|c|c|c|}
\hline & 1 & 2 & 3 & 4 & 5 & 6 & 7 & 8 & 9 & \begin{tabular}{|l|l|}
10 & 11 \\
\end{tabular} & \begin{tabular}{|l|l|}
12 & 13 \\
\end{tabular} & \begin{tabular}{l|l|}
3 & 14 \\
\end{tabular} & 15 & \begin{tabular}{|l|l|}
16 \\
\end{tabular} & \begin{tabular}{l|l|}
17 & 1 \\
\end{tabular} & \begin{tabular}{l|l}
18 & 19 \\
\end{tabular} & $\begin{array}{l}920 \\
\end{array}$ & 21 & 22 & \begin{tabular}{|l|l|}
23 & 24 \\
\end{tabular} & \begin{tabular}{l|l|}
4 & 25 \\
\end{tabular} & 26 & & 27 & 28 & 29 & 30 & 31 & 32 & 33 & 34 \\
\hline $\begin{array}{l}\text { Malus domestica } \\
\text { Borkh. }\end{array}$ & $:-$ & & & & & & -1 & -1 & & 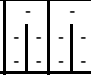 & 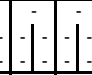 & & & & 1 & & & & & & it: & $\begin{array}{ll}i & : \\
1 & -\end{array}$ & & & 1 & 1 & 1 & Ke & hort. & $\mathrm{mf}$ & Robin \\
\hline Malva pusilla Smith & $-1:$ & & & & & & & & & $-1:$ & & & & & & & & & & & $\because:$ & & 1 & 1 & 1 & 1 & 1 & Ar & $\begin{array}{l}\text { |ranian-Turanian- } \\
\text { Euro-siber or } \\
\text { arch resist? }\end{array}$ & th & Stel medi \\
\hline Marrubium vulgare $\mathrm{L}$. & $\therefore$ & & & & & & & & & -1 & & & & & & & & $2]-$ & & & $\therefore:$ & \begin{tabular}{l|l}
1 & 1 \\
1 & 1
\end{tabular} & & \begin{tabular}{l|l|}
2 & 2 \\
2 & - \\
\end{tabular} & 1 & 3 & 6 & Ar & \begin{tabular}{l|}
$\begin{array}{l}\text { Mediterr-Iranian- } \\
\text { Turanian }\end{array}$ \\
\end{tabular} & ch & Artemi \\
\hline Matricaria recutita $\mathrm{L}$. & $-j$ & & & & & & & & & & & & & & & & & & & & $j$ & & 1. & \begin{tabular}{l|l|}
- & 1
\end{tabular} & 1 & 1 & 1 & Ar & E-Mediterr & $\mathrm{t}$ & Stel medi \\
\hline Medicago falcata L. & \begin{tabular}{|l|l|} 
& 1 \\
2 & 3 \\
2 & 2 \\
\end{tabular} & & & & & 1 & \begin{tabular}{|l|l|} 
& -1 \\
2 & 2 \\
2 & 2 \\
\end{tabular} & \begin{tabular}{|c|c|} 
& -1 \\
2 & 2 \\
2 & 3
\end{tabular} & & & & \begin{tabular}{|c|c|c} 
& 2 \\
2 & 3 & 2 \\
2 & 1
\end{tabular} & & & & & & & & & $-i-$ & \begin{tabular}{l|l}
16 & 18 \\
21 & 16
\end{tabular} & \begin{tabular}{l|l}
18 & 31 \\
19 & 43
\end{tabular} & \begin{tabular}{l|l|l|}
31 & 38 \\
43 & 36
\end{tabular} & 21 & 83 & 163 & $\mathrm{Ha}$ & & $\mathrm{h}$ & Fest-Brom, Gali veri \\
\hline Medicago lupulina L. & $-i$ & & & & & & -1 & -1 & & $-1 . j$ & & & & & & & & & & & 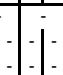 & \begin{tabular}{l|l}
2 & 1 \\
- & 3
\end{tabular} & \begin{tabular}{l|l}
1 & 2 \\
3 &
\end{tabular} & \begin{tabular}{l|l|}
2 & 1 \\
$\cdot$ & 4
\end{tabular} & 4 & 6 & 7 & $A p$ & & th & Stel medi \\
\hline $\begin{array}{l}\text { Medicago minima (L) } \\
\text { Bartal. }\end{array}$ & $\because:-1$ & & & & & & $-1:-1$ & $\begin{array}{ll}1 & 2 \\
1 & -1\end{array}$ & & -1 & & & & & & & & 1 & & & $\because:$ & \begin{tabular}{l|l}
1 & 1 \\
- & -
\end{tabular} & \begin{tabular}{l|l}
1 & 1 \\
- & -
\end{tabular} & \begin{tabular}{l|l|l} 
& 2
\end{tabular} & 1 & 2 & 3 & Ns & & $t$ & Fest-Brom, Poly-Arte \\
\hline $\begin{array}{l}\text { Melandrium album } \\
\text { (Mill.) Garcke }\end{array}$ & $i$ & & & & & & \begin{tabular}{|l|l|} 
& 1 \\
2 & 2 \\
2 & 2 \\
\end{tabular} & 1 & & & & & & & & & & & & & $i$ & & \begin{tabular}{l|l}
13 & 10 \\
17 & 27 \\
17
\end{tabular} & \begin{tabular}{|l|l|}
13 & 13 \\
10 & 16 \\
27 & 30 \\
\end{tabular} & 22 & 64 & 96 & $A p$ & & th & Artemi, Robin \\
\hline Melilotus albus Medik. & ( & & & & & & & & & & & & & & & & & & & & i. & & $2:$ & $:-13$ & 3 & 3 & 4 & $A p$ & & th & Artemi \\
\hline $\begin{array}{l}\text { Melilotus officinalis } \\
\text { (L.) Pall. }\end{array}$ & \begin{tabular}{|c|c|} 
& 2 \\
& 1 \\
2 & 1 \\
\end{tabular} & & $-\begin{array}{r}1 \\
2 \\
\end{array}$ & & 1 & 1] 2 & $-i \begin{array}{l}1 \\
1 \\
i\end{array}$ & $\begin{array}{ll}-1 \\
1 \\
1\end{array}$ & & \begin{tabular}{|c|c|c|}
2 & - \\
-1 & 3 & -1 \\
1 & 2 & 1 \\
\end{tabular} & & & & & & & & & & 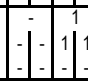 & $i$ & & \begin{tabular}{l|c}
13 & 4 \\
11 & 10
\end{tabular} & \begin{tabular}{c|c|}
4 & 17 \\
10 & 18 \\
\end{tabular} & 15 & 41 & 56 & $A p$ & & th & Artemi \\
\hline Morus alba L. & $i:$ & & & & & & $\therefore:$ & $\therefore$ & & -1 & & & & & $i$ & i & & - & & $i$ & $\because$ & $-j{ }_{2}^{1}$ & \begin{tabular}{l|l}
1 & \\
4 & 2
\end{tabular} & $i i_{4}$ & 6 & 7 & 7 & Ke & E-Asiatic & $\mathrm{mf}$ & Robin \\
\hline $\begin{array}{l}\text { Muscari neglectum } \\
\text { Guss. ex Ten. } \\
\end{array}$ & -1 & 1. & 1 & & 1 & & $\therefore$ & $j$ & & $i$ & 12 & 2 & & & 1 & 1 & & & & 1 & $1:$ & 2 & & \begin{tabular}{l|l|}
2 & 2 \\
\end{tabular} & 3 & 4 & 5 & \begin{tabular}{|c|}
$N s$ \\
$(* * * x)$ \\
\end{tabular} & & g & $\begin{array}{l}\text { Fest-Pucc, Gali veri, Fest- } \\
\text { Brom }\end{array}$ \\
\hline $\begin{array}{l}\text { Myosotis arvensis (L.) } \\
\text { Hill }\end{array}$ & $i$ & -1 & : & & 1 & & $\because \vdots$ & -1 & & is & 1 & & & & 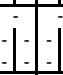 & $\frac{1}{2}$ & & -1 & & -1 & 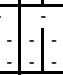 & & \begin{tabular}{l|l}
1 & 2 \\
3 & 2 \\
\end{tabular} & \begin{tabular}{l|l|}
2 & 2 \\
2 & 4 \\
\end{tabular} & 4 & 8 & 12 & $\mathrm{Ar}$ & \begin{tabular}{|l|}
$\begin{array}{l}\text { Mediterr-Iranian- } \\
\text { Turanian }\end{array}$ \\
\end{tabular} & th & Stel medi \\
\hline $\begin{array}{l}\text { Myosotis micrantha } \\
\text { Pall. ex Lehm. }\end{array}$ & $\because$ & & & & 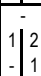 & & 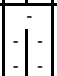 & . & & .. & & & & & -1 & & & & & $\left.\right|_{1} ^{1}$ & 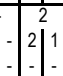 & & \begin{tabular}{l|l}
4 & 10 \\
1 &.
\end{tabular} & \begin{tabular}{l|l|}
10 & 5 \\
. & 1
\end{tabular} & 7 & 15 & 23 & $\mathrm{Ha}$ & & $t$ & $\begin{array}{l}\text { Fest vagi, Fest-Brom, } \\
\text { Poly-Arte }\end{array}$ \\
\hline Nepeta cataria L. & $\because-$ & & & & & & 1 & -1. & & $i$ & & & & & & & & & & it & $i_{2}$ & & \begin{tabular}{l|l} 
& \\
7 & 1 \\
\end{tabular} & \begin{tabular}{|l|l|}
1 & \\
& 1 \\
& 9 \\
\end{tabular} & 7 & 10 & 12 & $\mathrm{Ar}$ & E-Mediterr & ch & $\begin{array}{l}\text { Artemi, Urti-Samb, Rham- } \\
\text { Prun }\end{array}$ \\
\hline Nepeta pannonica $L$. & $\therefore$ & & & & & & $\because:$ & -1 & & $\therefore$ & $\because$ & & & & -1 & & & & & 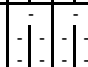 & $\because$ & $i^{1}$ & \begin{tabular}{l|l}
1 & 5 \\
\end{tabular} & \begin{tabular}{c|c|} 
& 1 \\
&
\end{tabular} & 2 & 2 & 4 & Ns & & ch & Fest-Brom, Gali-veri \\
\hline Nigella arvensis $\mathrm{L}$. & $i$ & & & & & & $\because$ & & & $\left|\begin{array}{lll}1 & 1 \\
2 & 1\end{array}\right|$ & -1 & & & & 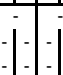 & & & & & 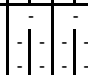 & $i$ & \begin{tabular}{l|l}
2 & 1 \\
2 & 1
\end{tabular} & \begin{tabular}{l|l}
1 & 2 \\
1 & 4
\end{tabular} & \begin{tabular}{l|l|}
2 & 1 \\
4 & 1
\end{tabular} & 2 & 6 & 8 & Ar & \begin{tabular}{|l}
$\begin{array}{l}\text { Balkan-W- } \\
\text { Asiatic }\end{array}$ \\
\end{tabular} & $t$ & Stel medi, [Fest-Brom] \\
\hline Nonea rossica Steven & $\begin{array}{ll}1 & 1 \\
1 & 1 \\
\end{array}$ & & $\mid \begin{array}{lll}1 \\
1\end{array}$ & & & & {$\left[\begin{array}{cc}1 \\
-1 \\
1\end{array}\right]$} & $\left|\begin{array}{l|l}1 & 2 \\
2 & 1\end{array}\right|$ & $1]$. & . & & & & & -1 & 1 & 1. & & & -1 & i. & 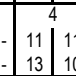 & \begin{tabular}{l|l}
11 & 14 \\
10 & 17
\end{tabular} & \begin{tabular}{l|l|l}
14 & 13 \\
17 & 10 \\
\end{tabular} & 17 & 49 & 59 & $\mathrm{Ha}$ & & $\mathrm{h}$ & Fest-Brom, Artemi \\
\hline $\begin{array}{l}\text { Oberna behen (L.) } \\
\text { Ikonn. }\end{array}$ & -1 & & -1 & $-1-1$ & $-1-$ & & $\therefore$ & -1. & & : & $1:$ & & & & \begin{tabular}{|l|l|} 
& 2 \\
1 & 1 \\
\end{tabular} & 1 & -1. & i. & - & $j$ & $j$ & & \begin{tabular}{l|l}
2 & 2 \\
& 1 \\
\end{tabular} & \begin{tabular}{|l|l|}
2 & 2 \\
1 & 2 \\
\end{tabular} & 3 & 5 & 7 & $\mathrm{Ha}$ & & $\mathrm{h}$ & Moli-Arrh, Artemi \\
\hline $\begin{array}{l}\text { Odontites luteus (L.) } \\
\text { Clairv. }\end{array}$ & $\therefore$ & $\because:$ & 1 & $\begin{array}{lll}2 & - \\
2 & -1 & 0\end{array}$ &. & & $: 1$ & 1 & & $\therefore$ & $1:$ & -1. & & & 1 & 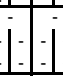 & 1 & & & : & D. & $\begin{array}{l}1 \\
1\end{array}$ & & \begin{tabular}{l|l|}
2 & $:$ \\
2 & $:$ \\
\end{tabular} & 1 & 2 & 4 & Ns & & $t$ & Fest-Brom \\
\hline $\begin{array}{l}\text { Odontites vulgaris } \\
\text { Moench }\end{array}$ & $-\begin{array}{l}1 \\
3 \\
\end{array}$ & & $1:$ & $\left.\mid \begin{array}{ll}2 \\
2\end{array}\right]$ & $1-$ & & : & $-\begin{array}{l}1 \\
3 \\
\end{array}$ & & $1:$ & -1 & \begin{tabular}{rl|l|l|l|l|l|l|}
2 & 2 \\
2
\end{tabular} & \begin{tabular}{|l|l|l|l|l|}
1 & 2 \\
3 & 3 \\
\end{tabular} & $\mid-1$ & $-\left|\begin{array}{ll}2 & -1 \\
2\end{array}\right|$ & $\mid \begin{array}{l}1 \\
2\end{array}$ & -1. & -1 & -1 & 1 & 1 & \begin{tabular}{l|l}
3 & 6 \\
3 & 8 \\
\end{tabular} & \begin{tabular}{l|l}
6 & 4 \\
8 & 7 \\
\end{tabular} & \begin{tabular}{|l|c|}
4 & 9 \\
7 & 18 \\
\end{tabular} & 8 & 21 & 40 & $\mathrm{Ha}$ & & $t$ & Moli-Arrh, Artemi \\
\hline $\begin{array}{l}\text { Omphalodes scorpio- } \\
\text { ides (Haenke) Schrank }\end{array}$ & $\therefore$ & $\therefore \frac{1}{2}$ & $\therefore:$ & $1-1$. & $1.1-$ &. & $\therefore$ & i. & 1 & $\therefore$ & 1. & -1 & 1. & -1 & 1.1 & $\therefore$ & $\therefore$ & 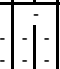 & 1 & jo & $\therefore$ & & i: & $\therefore:$ & 1 & 1 & 2 & Ns & & $\mathrm{h}$ & Quer-Fage \\
\hline $\begin{array}{l}\text { Onobrychis gracilis } \\
\text { Besser }\end{array}$ & \begin{tabular}{|l|l|}
1 & 2 \\
2 & 2 \\
\end{tabular} & : & i: & -1 & .1. & 1 & 11 & -1 & & $:$ & -1 & -1 & . & & $1:$ & $-i$ & 1 & 1. & & -1 & - & \begin{tabular}{|l|l|} 
& 1 \\
1 & 3 \\
2 & 1 \\
\end{tabular} & \begin{tabular}{l|l}
3 & 1 \\
1 & 3 \\
\end{tabular} & \begin{tabular}{l|l|}
1 & 4 \\
3 & 2 \\
\end{tabular} & 3 & 8 & 11 & Ns & & $\mathrm{h}$ & Fest-Brom \\
\hline
\end{tabular}




\begin{tabular}{|c|c|c|c|c|c|c|c|c|c|c|c|c|c|c|c|c|c|c|c|c|c|c|c|c|c|c|c|c|c|c|c|}
\hline & 1 & $2 \mid$ & 3 & 4 & 5 & 6 & 7 & 8 & 9 & \begin{tabular}{|l|l|}
10 & 11 \\
\end{tabular} & \begin{tabular}{|l|l|}
12 & 13 \\
1
\end{tabular} & 14 & 15 & \begin{tabular}{l|l}
16 & 17 \\
\end{tabular} & 18 & 19 & 20 & 21 & 22 & \begin{tabular}{|l|l|}
23 & 24 \\
1
\end{tabular} & \begin{tabular}{l|l|l|}
425 \\
\end{tabular} & $\frac{26}{3}$ & & $\frac{27}{2}$ & 28 & 29 & 30 & 31 & 32 & 33 & 34 \\
\hline $\begin{array}{l}\text { Onopordum } \\
\text { acanthium L. }\end{array}$ & $:-$ & & & & & & is & -1 & & $-\begin{array}{c}0 \\
-1\end{array}$ & & & & & & & & & & & $\begin{array}{lll}1 & - & - \\
1 & - & - \\
\end{array}$ & \begin{tabular}{|l|l|l|} 
& 3 \\
2 & 1 \\
4 & 4 \\
\end{tabular} & & \begin{tabular}{|l|l|}
2 & 3 \\
& 1 \\
\end{tabular} & 6 & 14 & 15 & Ar & \begin{tabular}{|l|}
$\begin{array}{l}\text { Mediterr-Iranian- } \\
\text { Turanian }\end{array}$ \\
\end{tabular} & $\mathrm{h}$ & Artemi \\
\hline Origanum vulgare $\mathrm{L}$. & 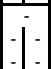 & & & & I2 1 & & $\because:$ & & & & & & & & & & & & & & -1 & \begin{tabular}{|l|l|}
3 & 5 \\
4 & 6 \\
\end{tabular} & & \begin{tabular}{l|l|}
4 & 7 \\
7 & 14
\end{tabular} & 7 & 19 & 33 & Ns & & ch & $\begin{array}{l}\begin{array}{l}\text { Gali veri, Trif-Gera, Quer } \\
\text { pube }\end{array} \\
\end{array}$ \\
\hline $\begin{array}{l}\text { Ornithogalum kochii } \\
\text { Parl. }\end{array}$ & $i$ & 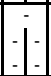 & & & -1 & & 1. & 1. & & $\therefore-1$ & $22^{2}-1$. & & & & & & & & & & \begin{tabular}{|l|l|l|}
2 & 2 & 2 \\
1 & 2 & 2 \\
1 & -1
\end{tabular} & & & \begin{tabular}{l|l|l}
5 & 6 \\
3 & 1 \\
\end{tabular} & 3 & 12 & 21 & Ns & & $\mathrm{g}$ & Fest-Brom \\
\hline $\begin{array}{l}\text { Otites densiflorum } \\
\text { (D`Urv.) Grossh. aggr. }\end{array}$ & $-j$ & & & & 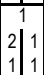 & & 1. & \begin{tabular}{|c|c|} 
& 2 \\
3 & 1 \\
-1 & 1
\end{tabular} & & \begin{tabular}{|l|l|l|}
2 & -1 \\
2 & 1 & 1 \\
2 & 1 & -1 \\
\end{tabular} & 1 & & & & & \begin{tabular}{c|c|c|}
2 & \\
3 & 2 \\
3 & 1
\end{tabular} \mid & & & & & $\begin{array}{|lll|}1 & 1 & - \\
1 & 1 & - \\
\end{array}$ & \begin{tabular}{|l|l|l|}
7 & 7 \\
7 & 7 \\
5 & 4 \\
\end{tabular} & & \begin{tabular}{l|l|}
\multicolumn{1}{|c|}{} & 8 \\
$8^{10}$ & 8 \\
\end{tabular} & 10 & 30 & 44 & Ns & & $\mathrm{h}$ & $\begin{array}{l}\text { Fest vagi, Fest-Brom, } \\
\text { Poly-Arte }\end{array}$ \\
\hline $\begin{array}{l}\text { Otites exaltatus (Friv.) } \\
\text { Holub }\end{array}$ & $\begin{array}{ll}-1 \\
1 \\
1 \\
\end{array}$ & & -1 & & - & & $: 1:$ & & & 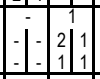 & & & & & & & & & & & $\therefore$ & \begin{tabular}{|l|l}
1 & 3 \\
3 & 3 \\
\end{tabular} & & \begin{tabular}{|l|l|}
2 & 3 \\
3 & 3 \\
\end{tabular} & 3 & 11 & 12 & Ns & & $\mathrm{h}$ & Fest-Brom \\
\hline Panicum miliaceum L. & -1 & & & & & & $i$ & & & & -1 & & & & & & & & & & -1 & \begin{tabular}{|l|l|}
$i$ & 2 \\
2 & 2
\end{tabular} & & \begin{tabular}{|l|l|} 
& 1 \\
\end{tabular} & 3 & 4 & 4 & $\mathrm{Ke}_{\mathrm{e}}$ & C-Asiatic & $\mathrm{t}$ & Stel medi \\
\hline $\begin{array}{l}\text { Persicaria lapathi- } \\
\text { folium (L) Delarbre }\end{array}$ & -1 & & & & - & & -1. & $: 1:$ & 1 & & & & -1 & & & & & & & & $\therefore$ & \begin{tabular}{|l|l} 
& $i$ \\
\end{tabular} & & $i|i|$ & 1 & 2 & 2 & $\mathrm{Ha}$ & & $\mathrm{t}$ & Bident, Stel medi \\
\hline $\begin{array}{l}\text { Peucedanum } \\
\text { alsaticum } \mathrm{L} .\end{array}$ & $:$ & & & & 1 & & 1. & -1 & & i. & $\therefore \begin{array}{l}2 \\
2 \\
2\end{array}$ & $: 1$ & & 1 & & - & & 1 & & \begin{tabular}{|l|l|} 
& -1 \\
2 & 2 \\
\end{tabular} & i & \begin{tabular}{|l|l|l|} 
& 2 & \\
2 & 2 \\
3 & 4 \\
\end{tabular} & & \begin{tabular}{|l|l|} 
& 1 \\
4 & 3 \\
6 & 9 \\
\end{tabular} & 4 & 13 & 24 & \begin{tabular}{|c|}
$N s$ \\
$(* * * *)$ \\
\end{tabular} & & $\mathrm{h}$ & Fest-Brom \\
\hline \begin{tabular}{|l} 
Phalacroloma \\
annuum (L.) Dumort.
\end{tabular} & $i$ & & - & i] & E & & -1. & & & -1. & & & & & & & & & & & $\therefore$ & \begin{tabular}{|l|l|}
$i$ & 1 \\
2 & 4 \\
\end{tabular} & & 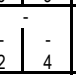 & 5 & 6 & 6 & Ke & North American & ht & Arte vulg, Gali veri \\
\hline $\begin{array}{l}\text { Phelipanche purpurea } \\
\text { (Jacq.) Sojảk }\end{array}$ & $i$ & & & & & & $i$ & & & $i$ & -1 & & & & & & & & & & -1 & $\left.1\right|^{2}$ & & $j 1$ & 1 & 2 & 2 & Ns & & $g$ & Fest-Brom, Artemi \\
\hline $\begin{array}{l}\text { Phleum phleoides (L.) } \\
\text { H.Karst. }\end{array}$ & 1 & &. & &. & & $:-$ & $:-1$ & & jar & \begin{tabular}{cc|c|c|}
-1 & 2 & -1 \\
\end{tabular} & .1. & 1 & & & {$\left[\begin{array}{lll}2 & 2 \\
2 & 2\end{array}\right.$} & & . & & .1. & $-2 \begin{array}{c}2 \\
2 \\
2\end{array}$ & \begin{tabular}{|l|l|l}
3 & 3 \\
3 & 3 \\
1 & 2 \\
\end{tabular} & & \begin{tabular}{l|l|}
6 & 5 \\
2 & 4 \\
\end{tabular} & 5 & 12 & 21 & Ns & & hg & Gali veri, Fest-Brom \\
\hline Phleum pratense $\mathrm{L}$. & $i$ & & & & & & $i$ & -1. & & -1 & 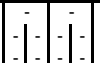 & 1.1 & & & & & & & & 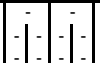 & $i$ & $-i_{1}^{1}$ & & $\mid \begin{array}{l}3 \\
2\end{array}$ & 1 & 2 & 5 & $\mathrm{Ha}$ & & gh & Moli-Arrh \\
\hline $\begin{array}{l}\text { Phlomis pungens } \\
\text { Willd. }\end{array}$ & i 15 & & & & & & $-1-$ & & & 1 & -1 & & & & & & & & & 1 & 1 & \begin{tabular}{|l|l|}
2 & 1 \\
3 & 2 \\
\end{tabular} & & \begin{tabular}{l|l|}
2 & 1 \\
4 & 2 \\
\end{tabular} & 3 & 8 & 9 & \begin{tabular}{|c|}
$N s$ \\
$(* * *)$ \\
\end{tabular} & & $\mathrm{h}$ & Fest-Brom \\
\hline Phlomis tuberosa L. & $i$ & & & & - & & $i$ & & & -1 & $\left.\left.2\right|^{2}\right|_{2} ^{2}$ & & & & & & & & & \begin{tabular}{|l|l|l|l|}
2 & 2 & 1 & 2 \\
2 & 3 & 2 & 3
\end{tabular} \mid & \begin{tabular}{|l|l|l|}
2 & 1 & \\
2 & 2 & 2 \\
& 2 & 2 \\
\end{tabular} & \begin{tabular}{|l|l|l}
7 & 6 \\
6 & 9 \\
\end{tabular} & & \begin{tabular}{l|l|l|}
1 & 12 \\
9 & 21
\end{tabular} & 10 & 31 & 56 & Ns & & hg & Fest-Brom, Gali veri \\
\hline 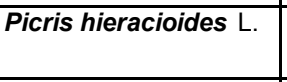 & -12 & & & & & & $i$ & \begin{tabular}{|r|r|}
-1 & 2 \\
2 & 2 \\
\end{tabular} & & 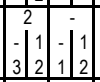 & -1 & & & & & & & 11 & & - & $i=$ & \begin{tabular}{|l|l|l|}
3 & 2 \\
3 & 10 \\
13 & 13 \\
\end{tabular} & & \begin{tabular}{|l|l|}
\multicolumn{1}{|c|}{} & 14 \\
5 & 14 \\
4 & 22 \\
\end{tabular} & 17 & 41 & 69 & $\mathrm{Ha}$ & & $\mathrm{h}$ & Fest vagi, Artemi \\
\hline $\begin{array}{l}\text { Pimpinella saxifraga } \\
\text { L. }\end{array}$ & 2 & -11 & & & - & & -1 & $i-1$. & & 1 & \begin{tabular}{r|r|}
-1 & -1 \\
& -1 \\
\end{tabular} & $-i_{2}^{1}$ & 1 & 1 & & $-i$ & i. & i. & & i. & $i$ & \begin{tabular}{|l|l|l} 
& 1 \\
$i$ & 3 \\
& 9 \\
\end{tabular} & & \begin{tabular}{|l|l|}
2 & 3 \\
2 & 13 \\
\end{tabular} & 9 & 14 & 19 & $\mathrm{Ha}$ & & $\mathrm{h}$ & \begin{tabular}{|l|} 
Fest-Brom, Artemi, Moli- \\
Arrh, Vacc-Pice
\end{tabular} \\
\hline Plantago lanceolata L. & $\begin{array}{c}-1 \\
1 \\
1\end{array}$ & & & & 11 & & . & & & -1. & -1. & & & & & & & & & & & \begin{tabular}{|l|l}
1 & 2 \\
4 & 4 \\
\end{tabular} & & \begin{tabular}{|l|l|}
1 & 2 \\
5 & 5 \\
\end{tabular} & 6 & 12 & 15 & $\mathrm{Ha}$ & & $\mathrm{h}$ & Gali veri, Fest-Brom, \\
\hline Plantago major L. & $i$ & & & -1 & & & $i$ & & & $\therefore$ & i. & $\because$ & & & & & & - & & 1. & j. & $i$ & & $i$ & 1 & 1 & 1 & $A p$ & & $\mathrm{~h}$ & Plan majo \\
\hline \begin{tabular}{|l|} 
Plantago urvillei Opiz \\
\end{tabular} & \begin{tabular}{|c|}
2 \\
-12 \\
1 \\
\end{tabular} & \begin{tabular}{|l|l|} 
& 1 \\
2 & 2 \\
-2 & 2 \\
\end{tabular} & & & & & - i. & & & -1 & \begin{tabular}{rl|l|}
- & 2 & 2 \\
-2 & 2 & 2 \\
\end{tabular} & - & & & & & & & & 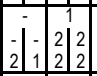 & 2 & \begin{tabular}{|l|l|l|}
5 & 5 \\
5 & 8 \\
5 & 8 \\
\end{tabular} & & \begin{tabular}{l|l|}
8 & 15 \\
9 & 16 \\
\end{tabular} & 11 & 31 & 54 & Ns & & $\mathrm{h}$ & Fest-Brom, Gali veri \\
\hline Poa angustifolia L. & \begin{tabular}{|l|l|} 
& -3 \\
2 & 3 \\
\end{tabular} & \begin{tabular}{r|r|}
$-i$ & 2 \\
2 & 2 \\
\end{tabular} & & & -3 & \begin{tabular}{|l|l|} 
& 2 \\
1 & 3 \\
2 & 3 \\
\end{tabular} & \begin{tabular}{|l|l|l|} 
& 2 \\
2 & 3 \\
2 & 3 \\
\end{tabular} & \begin{tabular}{|l|l|}
2 & 3 \\
3 & 3 \\
\end{tabular} & \begin{tabular}{|l|l|}
$\begin{array}{l}2 \\
2\end{array}$ & 2 \\
3 & 3 \\
\end{tabular} & \begin{tabular}{|c|c|c|c|}
2 & 2 & 2 & 2 \\
3 & 1 & 3 & 3 \\
\end{tabular} & 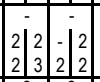 & \begin{tabular}{|l|l|} 
& 1 \\
2 & 3 \\
2 & 3 \\
\end{tabular} & \begin{tabular}{|l|l|l|} 
& 2 \\
2 & 3 \\
2 & 3 \\
\end{tabular} & \begin{tabular}{|l|l|l|}
2 & 2 \\
2 & 2 & 2 \\
3 & 3 & 2 \\
\end{tabular} & \begin{tabular}{|l|l|l|}
2 & 2 & 2 \\
3 & 2 & 2 \\
\end{tabular} & \begin{tabular}{|l|l|l|}
2 & 2 \\
2 & 2 & 2 \\
3 & 2 & 2 \\
\end{tabular} & \begin{tabular}{|l|l|l|} 
& 3 \\
3 & 3 \\
\end{tabular} & \begin{tabular}{|l|l|}
3 & 3 \\
3 & 3 \\
3 & 3 \\
\end{tabular} & $\begin{array}{l}2 \\
3 \\
\end{array}$ & \begin{tabular}{|l|l|l|l|}
-1 & 2 & 2 & 3 \\
3 & 3 & 3 & 3 \\
\end{tabular} & \begin{tabular}{|l|l|l|}
3 & 1 & 2 \\
& 2 & 2 \\
3 & 2 \\
\end{tabular} & \begin{tabular}{|l|l|}
16 & 16 \\
21 & 25 \\
24 & 24 \\
\end{tabular} & & \begin{tabular}{|l|l|}
33 & 33 \\
2 & 63 \\
2 & 65 \\
\end{tabular} & 25 & 110 & 265 & $\mathrm{Ha}$ & & gh & \begin{tabular}{|l|}
$\begin{array}{l}\text { Gali veri, Fest-Brom, Agro } \\
\text { int-rep, Robini }\end{array}$ \\
\end{tabular} \\
\hline Poa bulbosa L. & -1 & 2. & & & & & $\begin{array}{r}1 \\
-2 \\
-1\end{array}$ & & & $\begin{array}{ccc}-1 \\
-1\end{array}$ & 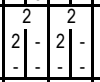 & 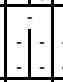 & & & & $\mid \begin{array}{rrr}1 & \\
2 & 1 & \\
2 & 1\end{array}$ & , & & & \begin{tabular}{|l|l|l|l|}
3 & 3 & 3 \\
3 & 2 & 2 & 2 \\
1 & 1 & 1 & 1 \\
\end{tabular} & \begin{tabular}{|l|l|l|}
2 & 2 & 2 \\
1 & 2 & 2 \\
& -1 & \\
\end{tabular} & \begin{tabular}{|l|l|}
8 & 6 \\
8 & 6 \\
3 & 3 \\
\end{tabular} & & \begin{tabular}{|c|c|}
6 & 10 \\
6 & 4 \\
\end{tabular} & 11 & 28 & 53 & $\mathrm{Ha}$ & & $\mathrm{h}$ & \begin{tabular}{|l} 
Fest-Brom, Poly-Arte, \\
Fest vagi, Agr int-rep
\end{tabular} \\
\hline Poa compressa $\mathrm{L}$. & $\because$ & $1-1$. & & $\begin{array}{lll}-1 \\
1\end{array} \mid$ & & $1-1-$ & - & $-1-1$ & & - & 1 & 2 & 1]2 & & & & -1 & & & $: 0$ & 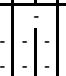 & \begin{tabular}{|l|l}
$-i$ & \\
2 & 6
\end{tabular} & & \begin{tabular}{ll|}
- & -12 \\
&
\end{tabular} & 7 & 8 & 14 & $\mathrm{Ha}$ & & $\mathrm{h}$ & Fest-Brom, Agro int-rep \\
\hline Poa nemoralis $\mathrm{L}$. & -1. & & & $i_{2}^{-} \mid \frac{1}{2}$ & & & -1 & $-i-$ & & i: & 1 & -1 & $i$ & & & -1 & -1 & 1 & -1 & -1. & -1 & i 1 & & $\dot{i} i_{2}$ & 1 & 2 & 4 & $\mathrm{Ha}$ & & $\mathrm{h}$ & Quer-Fage \\
\hline
\end{tabular}




\begin{tabular}{|c|c|c|c|c|c|c|c|c|c|c|c|c|c|c|c|c|c|c|c|c|c|c|c|c|c|c|c|c|c|c|}
\hline & 1 & 2 & 3 & 4 & 5 & 6 & 7 & 8 & & \begin{tabular}{|l|l|}
10 & 11 \\
\end{tabular} & \begin{tabular}{|l|l|}
12 & 13 \\
\end{tabular} & 14 & 15 & \begin{tabular}{l|l}
16 & 1 \\
\end{tabular} & \begin{tabular}{|l|l|}
17 & 18 \\
\end{tabular} & \begin{tabular}{l|l|}
8 & 19 \\
\end{tabular} & 20 & 21 & 22 & \begin{tabular}{|l|l|}
23 & 24 \\
\end{tabular} & \begin{tabular}{l|l|r|r|r|r|}
425 \\
\end{tabular} & 26 & 27 & 28 & 29 & 30 & 31 & 32 & 33 & 34 \\
\hline Poa pratensis $\mathrm{L}$. & -1. & & & & & & i. & & & -1. & 101 & & & & & & & & & & $-1-$ & & & 1 & 1 & 1 & $\mathrm{Ha}$ & & g & Moli-Arrh \\
\hline $\begin{array}{l}\text { Polygala comosa } \\
\text { Schkuhr }\end{array}$ & 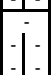 & & 10 & -1. & 1 & & $: 1$ & & & & $1=$ & & & & & $-1:$ & & & & $-1:-10$ & $-i$ & \begin{tabular}{lll|}
$:$ & 1 \\
- & -1
\end{tabular} & $-\mid \begin{array}{l}1 \\
-\end{array}$ & 1 & 1 & 1 & Ns & & $\mathrm{h}$ & Fest-Brom \\
\hline $\begin{array}{l}\text { Polygonum aviculare } \\
\text { L. }\end{array}$ & -1. & & & & -1 & & .1 & & & & & & & & & 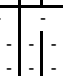 & & & & & -1 & \begin{tabular}{|l|l|} 
& - \\
2 & 2 \\
\end{tabular} & & 3 & 4 & 6 & $A p$ & & $\mathrm{t}$ & Poly-Arte, Plan majo \\
\hline \begin{tabular}{|l|} 
Polygonum \\
arenastrum Boreau
\end{tabular} & $\therefore$ & & & & & & $i$ & & & & & & & & & $j$ & & & & & -1 & $: i_{2}$ & & 2 & 2 & 3 & $A p$ & & $\mathrm{t}$ & Poly-Arte, Plan majo \\
\hline $\begin{array}{l}\text { Polygonum patulum } \\
\text { M.Bieb. }\end{array}$ & $-i$ & & & & 工 & & -1 & 2 & & 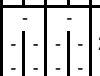 & 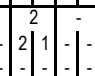 & & & & & 工 & & & & & -1. & \begin{tabular}{|l|l|} 
& 3 \\
& 3 \\
\end{tabular} & \begin{tabular}{|l|l|}
7 & 4 \\
& 3 \\
& 1 \\
\end{tabular} & 7 & 11 & 15 & $\mathrm{Ha}$ & & $\mathrm{t}$ & $\begin{array}{l}\text { Fest-Brom, Poly-Arte, } \\
\text { Artemi }\end{array}$ \\
\hline Populus alba L. & $i$ & & & & & & $i$ & & & $-1-1$ & $-j$ & & & & \begin{tabular}{l|l|l|} 
& 1 & -1 \\
1 & 1 & 1
\end{tabular} & -1 & & & & $-j$ & -1 & $-j_{1} 1$ & $-\left|\begin{array}{l}1 \\
1\end{array}\right|$ & 1 & 2 & 2 & $A p$ & & $\mathrm{mf}$ & Sali purp, Robini \\
\hline Portulaca oleracea L. & $\therefore$ & & & & & & i. & & & $\therefore$ & $\because:$ & & & & & 1 & & -1 & & $\therefore$ & $\therefore$ & $:-i_{1}$ & $:-i_{1}$ & 1 & 1 & 1 & Ar? & \begin{tabular}{|l|} 
Mediterr-sub- \\
Mediterr
\end{tabular} & $t$ & Stel medi \\
\hline Potentilla argentea $\mathrm{L}$. & \begin{tabular}{|l|l|}
1 & 2 \\
2 & 2 \\
\end{tabular} & & 1. & & & & $i$ & 12 & & & \begin{tabular}{|l|l|l|} 
& & \\
2 & 1 & \\
2 & 1 \\
\end{tabular} & & & & & \begin{tabular}{|c|c|} 
& 2 \\
3 & 2 \\
2 & 1 \\
\end{tabular} & & & & & 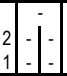 & \begin{tabular}{|l|l|}
8 & 5 \\
10 & 9 \\
\end{tabular} & \begin{tabular}{|l|l|}
\multicolumn{2}{|c|}{6} \\
14 & 11 \\
15 & 14 \\
\end{tabular} & 13 & 39 & 60 & $\mathrm{Ha}$ & & $\mathrm{h}$ & \begin{tabular}{|l} 
Gali veri, Fest vagi, Fest- \\
Brom, Artemi, Sedo-Scle
\end{tabular} \\
\hline $\begin{array}{l}\text { Potentilla canescens } \\
\text { Besser }\end{array}$ & $\therefore$ & & 11 & & 1 & & $i$ & -1 & & $\therefore-1$ & $\left.\begin{array}{|l|l|} & 2 \\
1 & 2 \\
1\end{array}\right]$. & -1 & & & . & {$\left[\begin{array}{l}1 \\
1 \\
1\end{array}\right]$} & & & & & i. & \begin{tabular}{|l|l|}
2 & 1 \\
2 & 2 \\
\end{tabular} & \begin{tabular}{|l|l|}
2 & 3 \\
2 & 1 \\
\end{tabular} & 3 & 8 & 9 & $\mathrm{Ha}$ & & $\mathrm{h}$ & Fest-Brom \\
\hline $\begin{array}{l}\text { Potentilla heptaphylla } \\
\text { L. }\end{array}$ & $:-1:$ & & 11 & $-:$ & 1 & 1 & $1:$ & : & & -1 & \begin{tabular}{|l|l|} 
& 2 \\
1 & 1 \\
\end{tabular} & 1 & & & . & -1 & & & & & -1 & \begin{tabular}{|l|l|l|}
1 & 2 \\
1 & 1 \\
\end{tabular} & \begin{tabular}{|l|l|}
1 & 3 \\
1 & 1 \\
\end{tabular} & 2 & 5 & 6 & Ns & & $\mathrm{h}$ & Fest-Brom \\
\hline \begin{tabular}{|l} 
Potentilla incana \\
P.Gaertn., B.Mey. \& \\
\end{tabular} & $i:$ & & $i$ & $i$ & 1 & & -1 & -1 & & $i$ & $i$ & & & & & \begin{tabular}{|l|l|} 
& 2 \\
2 & 2 \\
2 & 2 \\
\end{tabular} & & & & -1 & 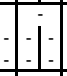 & \begin{tabular}{|l|l|}
2 & 2 \\
1 & 2 \\
\end{tabular} & \begin{tabular}{|l|l|}
4 & 4 \\
2 & - \\
\end{tabular} & 2 & 7 & 14 & Ns & & $\mathrm{h}$ & Fest-Brom, Fest vagi \\
\hline $\begin{array}{l}\text { Potentilla neglecta } \\
\text { Baumg. }\end{array}$ & 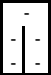 & & 1 & $i$ & & & - & & & $\begin{array}{l}1 \\
1\end{array} \mid:-$ & $\left.\mid \begin{array}{l}1 \\
1 \\
1\end{array}\right]$ & & & & & 1 & & & & -1 & $\therefore:$ & \begin{tabular}{|l|l|}
2 & 1 \\
4 & - \\
\end{tabular} & \begin{tabular}{|l|l|}
2 & 1 \\
4 & - \\
\end{tabular} & 5 & 8 & 8 & $\mathrm{Ha}$ & & $\mathrm{h}$ & Fest-Brom, Artemi \\
\hline \begin{tabular}{|l|} 
Potentilla patula \\
Waldst. et Kit.
\end{tabular} & \begin{tabular}{ll|}
2 & 2 \\
1 & 1 \\
\end{tabular} & & 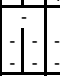 & & & & \begin{tabular}{ll|l}
1 & 1 \\
\end{tabular} & & & -1 & 15 & & & & & 1 & & & & & $i$ & \begin{tabular}{|l|l|}
2 & 2 \\
1 & 1 \\
\end{tabular} & \begin{tabular}{|l|l|}
3 & 3 \\
1 & 1 \\
\end{tabular} & 2 & 6 & 8 & Ns & & $\mathrm{h}$ & Fest-Brom \\
\hline Potentilla recta L. & $i$ & & & & & & & & & & -1 & & & & & & & & & & $i$ & 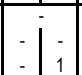 & $:-\mid i$ & 1 & 1 & 1 & Ns & & $\mathrm{h}$ & Fest-Brom \\
\hline $\begin{array}{l}\text { Prunus divaricata } \\
\text { Ledeb. }\end{array}$ & $i:$ & 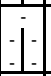 & $\because:-1$ & : & . & - 1 & . & $\therefore$ & & $-1:-1$. & $\therefore:-1:-1$ & 1 & & & (.t. & $1:$ & & -1 & & 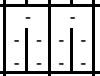 & $1:$ & \begin{tabular}{l|l}
- & 1 \\
\end{tabular} & \begin{tabular}{l|l|}
$:$ & $i$ \\
\end{tabular} & 1 & 1 & 1 & Ke & $\begin{array}{l}\text { SW-Asiatic, } \\
\text { Caucasian }\end{array}$ & nf & Robin \\
\hline Prunus domestica L. & $i=$ & & -1. & & 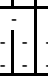 & 工 & 1 & -1 & & & $i$ & I & & & 1 & -1 & & & & & $i$ & & \begin{tabular}{|l|l|}
2 & - \\
4 & 1 \\
\end{tabular} & 4 & 7 & 8 & $E g$ & $\begin{array}{l}\text { SW-Asiatic, } \\
\text { Caucasian }\end{array}$ & $\mathrm{mf}$ & Robin \\
\hline $\begin{array}{l}\text { Prunus stepposa } \\
\text { Kotov }\end{array}$ & $i$ & $-i$ & {$[1$} & 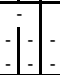 & & & & & & is & .1 & & & & & & & & & 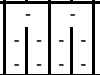 & $\left|\begin{array}{l|l}2 & 2 \\
2 & 3\end{array}\right|$ & \begin{tabular}{|l|l|}
1 & 1 \\
4 & 3 \\
\end{tabular} & \begin{tabular}{|l|l|}
2 & 2 \\
5 & 5 \\
\end{tabular} & 6 & 9 & 14 & Ns & & nf & Rham-Prun \\
\hline \begin{tabular}{|l} 
Psammophiliella \\
muralis (L.) Ikonn.
\end{tabular} & 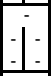 & 1 & . & i|:- & & 1 & -1 & 1 & & -1 & $1:$ & & 1 & & : & $:$ & & & & $: 0$ & -1 & \begin{tabular}{|l|l|} 
& $i$ \\
\end{tabular} & \begin{tabular}{|l|l|} 
& - \\
\end{tabular} & 1 & 2 & 2 & $\mathrm{Ha}$ & & $\mathrm{t}$ & Sedo-Scle, Stel medi \\
\hline Ptelea trifoliata L. & $i$ & & & & & -1 & & i. & & i & -1 & & & & 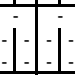 & 1 & & & & 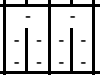 & $i$ & & $: i_{1}$ & 1 & 1 & 1 & Ke & \begin{tabular}{|l|} 
E-North \\
American
\end{tabular} & $\mathrm{mf}$ & Robin \\
\hline $\begin{array}{l}\text { Pulsatilla pratensis } \\
\text { (L.) Mill. }\end{array}$ & $:-1$ & 1 & I & 1. & 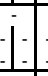 & 1. & 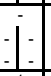 & 1 & & i: & 1 & & 1 & -1 & 1 & $-\begin{array}{l}2 \\
2\end{array}$ & 1 & & -1 & 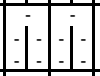 & $i$ & \begin{tabular}{l|l|l|}
2 & 1 \\
1 & 1 \\
\end{tabular} & \begin{tabular}{|l|l|}
3 & 1 \\
2 & 1 \\
\end{tabular} & 3 & 7 & 9 & \begin{tabular}{|c|}
$N s$ \\
$(* * *)$ \\
\end{tabular} & & $\mathrm{h}$ & Fest-Brom, Dicr-Pini \\
\hline Pyrus communis $\mathrm{L}$. & il: & & -1 & & & & & & & il & DII & & & 2| & & & $i$ & & & $i$ & $\left.\begin{array}{l}1 \\
1\end{array}\right]$ & \begin{tabular}{|l|c|} 
& 2 \\
4 & -1 \\
9 & 10 \\
\end{tabular} & \begin{tabular}{|l|l|}
5 & \\
9 & 11 \\
\end{tabular} & 17 & 25 & 27 & $A e$ & & $\mathrm{mf}$ & Robin, [Quer-Fage] \\
\hline Quercus robur $\mathrm{L}$. & $:-1:$ & $:-1$ & $\because$ & il: & 1 & $:$ & 1. & $\therefore$ & & $\begin{array}{lll} & 1 & 1 \\
\end{array}$ & $\therefore$ & -1 & 1.1 & 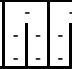 & 1 & ij & 1 & 1] & -1 & -1 & i & \begin{tabular}{|l|l|} 
& 1 \\
& 1 \\
\end{tabular} & \begin{tabular}{|l|l|} 
& 1 \\
& 1 \\
\end{tabular} & 5 & 8 & 9 & Ns & & $\mathrm{mf}$ & Quer-Fage \\
\hline $\begin{array}{l}\text { Ranunculus } \\
\text { oxyspermus Willd. }\end{array}$ & -1 & 1 & 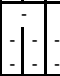 & 1 & & 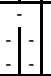 & & & & 1 & $1-1$ & -1 & & 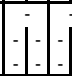 & 1 & 1 & - & -1. & & 1 & -1. & \begin{tabular}{l|l}
1 & - \\
&
\end{tabular} & \begin{tabular}{l|l}
1 & $:$ \\
& - \\
\end{tabular} & 1 & 1 & 1 & Ns & & $\mathrm{hg}$ & Fest-Brom \\
\hline
\end{tabular}




\begin{tabular}{|c|c|c|c|c|c|c|c|c|c|c|c|c|c|c|c|c|c|c|c|c|c|c|c|c|c|c|c|c|c|c|c|c|c|c|}
\hline & 1 & 2 & 3 & 4 & \begin{tabular}{|l|l|}
5 & 6 \\
1
\end{tabular} & 7 & 8 & 9 & 10 & 11 & 12 & & \begin{tabular}{|l|}
14 \\
\end{tabular} & 15 & \begin{tabular}{|l|}
16 \\
\end{tabular} & 17 & 18 & \begin{tabular}{|l|l|}
19 & 2 \\
\end{tabular} & \begin{tabular}{l|l}
20 & 21 \\
\end{tabular} & \begin{tabular}{|l|l|}
1 & 22 \\
\end{tabular} & 223 & 24 & 25 & & 26 & & $\frac{21}{1}$ & 28 & 29 & 30 & 31 & 32 & 33 & 34 \\
\hline \begin{tabular}{|l|} 
Ranunculus \\
polyanthemos L.
\end{tabular} & 2 & & & & & & & -1 & & & & & & & & & & & & & & & -1. & & \begin{tabular}{|l}
1 \\
3 \\
\end{tabular} & & \begin{tabular}{|l|}
1 \\
4 \\
\end{tabular} & 6 & 6 & 8 & Ns & & $\mathrm{hg}$ & Gali veri, Fest-Brom \\
\hline $\begin{array}{l}\text { Ranunculus scythicus } \\
\text { Klokov }\end{array}$ & $\begin{array}{ll}2 \\
-1\end{array}$ & & & & & 1.1 & & - & & & & & & & & & & -1 & & & 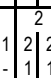 & & 2 & & $\begin{array}{l}10 \\
4 \\
4\end{array}$ & & $\mid \begin{array}{c}16 \\
5\end{array}$ & 11 & 26 & 38 & Ns & & gh & Fest-Brom \\
\hline \begin{tabular}{|l} 
Raphanus \\
raphanistrum L.
\end{tabular} & 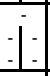 & & - & & . & $: 1$ & - & $: 1:$ & & & & 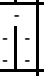 & & & & & & $\therefore:-1$ & & $-1:$ & 1 & 10 & 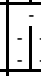 & & i & & $\perp_{1}$ & 1 & 1 & 1 & Ar & Mediterr & $\mathrm{t}$ & Stel medi \\
\hline Reseda lutea L. & 1. & & & & & & & & & & & & & & & & & & & & & & 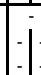 & & $i_{1}^{1}$ & & & 5 & 9 & 9 & Ke & Mediterr & th & $\begin{array}{l}\text { Stel medi, Artemi, Agro int } \\
\text { rep }\end{array}$ \\
\hline $\begin{array}{l}\text { Rhamnus cathartica } \\
\text { L. }\end{array}$ & 1 & & & & & -. & & $-1:$ & & & & & & & & & & & & & & & 1 & & \begin{tabular}{|l|}
4 \\
6 \\
\end{tabular} & & \begin{tabular}{|c|}
5 \\
12 \\
\end{tabular} & 8 & 22 & 30 & Ns & & nf & Rham-Prun \\
\hline $\begin{array}{l}\text { Robinia pseudoacacia } \\
\text { L. }\end{array}$ & $i$ & & & & & $i$ & & -1 & & & & & & & & & 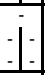 & 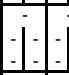 & & -12 & 2. & & $i$ & & $\mid \begin{array}{l}1 \\
1 \\
1\end{array}$ & & $\mid \begin{array}{l}2 \\
2 \\
2\end{array}$ & 1 & 4 & 8 & Ke & North American & $\mathrm{mf}$ & Robin \\
\hline Rosa canina L. & -1 & & & & & & & $\left.1\right|_{1} ^{1}$ & & & & & & & & & & $i$ & & & & & 1 & & $\mid \begin{array}{l}1 \\
2\end{array}$ & & $\left|\begin{array}{l}1 \\
2\end{array}\right|$ & 2 & 5 & 5 & Ns & & nf & Rham-Prun, Quer pub-pe \\
\hline $\begin{array}{l}\text { Rosa corymbifera } \\
\text { Borkh. } \\
\end{array}$ & $: 1:$ & $\mid-1$ & -1 & & -1 & -1 & $1-1$ & $i$ & $1:-1$ & & 1 & 1 & $\because$ & & -1 & - & $: 1:$ & $\therefore:-1$ & & $:$ & 1 & -1 & -1 & & i & & $i_{2}$ & 2 & 2 & 2 & Ns & & nf & Rham-Prun \\
\hline Rosa villosa $L$. & $1:$ & & & & & & & 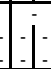 & & & & & & & & & & & & & & & -1 & & $i$ & & & 1 & 1 & 1 & Ns & & nf & Rham-Prun \\
\hline Rubus caesius $\mathrm{L}$. & $\therefore:$ & & & & & & & $i$ & $2 \sqrt{2}$ & & & & 3 & & & & & -1 & & & -1 & & $i$ & & \begin{tabular}{|l}
1 \\
1 \\
1
\end{tabular} & & $\mid \begin{array}{l}1 \\
2 \\
\end{array}$ & 2 & 4 & 8 & Ns & & nf & \begin{tabular}{|l}
$\begin{array}{l}\text { Sali purp, Gali-Urti, } \\
\text { Artemi }\end{array}$ \\
\end{tabular} \\
\hline $\begin{array}{l}\text { Rumex confertus } \\
\text { Willd. } \\
\end{array}$ & : & 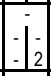 & & & & : & & $-i$ & & & & & -1 & & & & -1 & 1 & & & 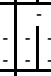 & & $i$ & & $I_{2}$ & & $i_{3}$ & 2 & 2 & 3 & $\mathrm{Ha}$ & & $\mathrm{h}$ & Moli-Arrh, Artemi \\
\hline Rumex crispus $\mathrm{L}$. & -1 & & & & & 1. & & -1 & & & & & & & & &. & & & & & & -1 & & \begin{tabular}{|l}
2 \\
4
\end{tabular} & & $\begin{array}{l}2 \\
4\end{array}$ & 4 & 8 & 8 & $A p$ & & $\mathrm{~h}$ & Moli-Arrh, Artemi \\
\hline Rumex patientia $\mathrm{L}$. & -1 & & & & & & & $i$. & & & & & & & & & i & & & & & & -1 & & $\begin{array}{l}2 \\
1 \\
1 \\
\end{array}$ & & $\begin{array}{l}2 \\
1 \\
\end{array}$ & 3 & 6 & 7 & $\mathrm{Ke}$ & $\begin{array}{l}\begin{array}{l}\text { ES-Mediterr- } \\
\text { continent }\end{array} \\
\end{array}$ & $\mathrm{h}$ & Artemi \\
\hline Salsola tragus $\mathrm{L}$. & & & & & & & & & & & & & & & & & & & & & & & -1 & & $\begin{array}{l}\vdots \\
1\end{array}$ & & $\begin{array}{l}\vdots \\
1 \\
\end{array}$ & 1 & 1 & 1 & $A p$ & & $\mathrm{t}$ & Stel medi, Caki mari \\
\hline Salvia aethiopis $\mathrm{L}$. & -1 & & & & & & & $i$ & & & & & $1-1$ & & & & -1 & $1-1$ & & & 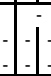 & & $i$ & & i & & $\mid \dot{2}$ & 1 & 2 & 3 & Ns & & $\mathrm{h}$ & Fest-Brom, Artemi \\
\hline Salvia austriaca Jacq. & $:-1$ & & & & i. & $1:$ & & $i$ & & & -1 & &.$j$ & & -1 & & - & -1 & & & 1 & & -1 & & i: & & : & 1 & 1 & 1 & $\begin{array}{c}N s \\
(* * *) \\
\end{array}$ & & $\mathrm{h}$ & Fest-Brom \\
\hline $\begin{array}{l}\text { Salvia } \mathrm{x} \\
\text { betonicaefolia Ett. }\end{array}$ & : & -1 & -1 & & 1 & i & & $\because:$ & 1 & & -1 & & -1 & & -1 & -1 & -1 & -1 & & & & & -1 & & $\begin{array}{l}1 \\
2 \\
\end{array}$ & & $\begin{array}{l}1 \\
2 \\
\end{array}$ & 3 & 5 & 5 & \begin{tabular}{c|}
$N s$ \\
$\left({ }^{* \star \star *}\right)$ \\
\end{tabular} & & $\mathrm{h}$ & Fest-Brom \\
\hline Salvia nemorosa L. & 11 & \begin{tabular}{|l|l|}
2 & 2 \\
3 & 2 \\
\end{tabular} & \begin{tabular}{|l|l|l|}
3 & 2 \\
2 & 2 \\
2
\end{tabular} & & \begin{tabular}{l|l|l|l|l}
2 & 2 \\
2 & 3 & 2 & 2 \\
2 & 2 & 2 & 2 \\
2
\end{tabular} & $\begin{array}{l}2-1 \\
-2 \\
2\end{array}$ & 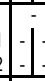 & \begin{tabular}{|l|l|}
-1 & 2 \\
2 & 2 \\
\end{tabular} & \begin{tabular}{|l|l|l|l|l|}
2 & 2 \\
3 & 2 \\
\end{tabular} & & $\begin{array}{lll}2 & 2 \\
2 & 2 & 2 \\
2 & 2\end{array}$ & $\begin{array}{l}2 \\
2 \\
\end{array}$ & \begin{tabular}{|l|l|l|} 
& 2 & 2 \\
2 & 2 \\
\end{tabular} & & 2 & \begin{tabular}{l|l|}
1 & 2 \\
2 & 2 \\
\end{tabular} & \begin{tabular}{|l|l|} 
& 1 \\
2 & 2 \\
& 2 \\
\end{tabular} & $\begin{array}{r}-1 \\
1 \\
2 \\
\end{array}$ & & \begin{tabular}{|l|l|l|l}
1 & 2 & 2 \\
2 & 2 & 1 \\
\end{tabular} & \begin{tabular}{l|l|l|l|l}
2 & 1 & 2 \\
1 & 2 & 2 &
\end{tabular} & \begin{tabular}{l|l|l}
2 & 1 & 3 \\
2 & 2 & 2 \\
\end{tabular} & $\begin{array}{lll} & 1 \\
2 & 2 \\
2 & 1 \\
\end{array}$ & & $\mid \begin{array}{l}21 \\
22\end{array}$ & & \begin{tabular}{|l|}
40 \\
41 \\
\end{tabular} & 22 & 92 & 168 & Ns & & $\mathrm{h}$ & Fest-Brom, Agro int-rep \\
\hline Salvia nutans $\mathrm{L}$. & \begin{tabular}{|l|l|} 
& 2 \\
1 & 2 \\
1 & 2 \\
\end{tabular} & $\because:-1$ & & & ij & \begin{tabular}{c|c|c|}
2 & 2 \\
2 & 2 \\
2 & 2 \\
\end{tabular} & 1 & $i$ & -1 & & -1. & 1 & 1 & & 1 & -1. & i. & 1): & & & .1 & & $i$ & & \begin{tabular}{|l}
2 \\
2 \\
3
\end{tabular} & & \begin{tabular}{|l|}
4 \\
5 \\
\end{tabular} & 4 & 12 & 19 & \begin{tabular}{|c|}
$N s$ \\
$\left({ }^{* * * *)}\right.$ \\
\end{tabular} & & $\mathrm{h}$ & Fest-Brom \\
\hline Salvia pratensis $L$. & 04 & & & & & $i$ & 1 & $i$ & 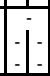 & & 工 & - & i & & & & 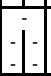 & $\therefore$ & & 11 & -1 & & -1. & & $i^{3}$ & & $\begin{array}{l}3 \\
\end{array}$ & 3 & 4 & 4 & Ns & & $\mathrm{h}$ & Fest-Brom \\
\hline Salvia verticillata $L$. & \begin{tabular}{|l|l|}
$i$ & 2 \\
1 & 2 \\
\end{tabular} & & & & 1 & -1. & & -1 & 1 & & $i$ & & -1 & & 1 & & & 1 & & & i. & & 1 & & \begin{tabular}{|l}
1 \\
1 \\
\end{tabular} & & \begin{tabular}{|l|}
2 \\
2 \\
\end{tabular} & 1 & 3 & 5 & $\mathrm{Ha}$ & & hg & \begin{tabular}{|l|}
$\begin{array}{l}\text { Fest-Brom, Gali veri, Agro } \\
\text { int-rep }\end{array}$ \\
\end{tabular} \\
\hline Sambucus nigra $\mathrm{L}$. & $\therefore$ & -12 & -1 & & i & $\begin{aligned} 2 \\
-1 \\
-1\end{aligned}$ & & 1 & & & -1 & & $1-1$ & 1] & & & -1 & -1 & & & & & 1 & & $\mid \begin{array}{l}1 \\
8\end{array}$ & & $\mid \begin{array}{c}1 \\
10\end{array}$ & 11 & 14 & 17 & $A p$ & & $\mathrm{nf}$ & Urti-Samb, Robini \\
\hline $\begin{array}{l}\text { Sambucus racemosa } \\
\text { L. }\end{array}$ & $1:$ & -1. & -1 & & $-1 .-1$. & -1 & 1 & $-1-$ & -1 & & -1 & I & -1 & & -1. & 1 & -1 & 1. & 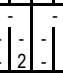 & $j$ & -1 & -1. & $\therefore$ & & $i$ & & $\mid \begin{array}{l}2 \\
\end{array}$ & 1 & 1 & 2 & Ns & & nf & Robin, Epil angu \\
\hline
\end{tabular}




\begin{tabular}{|c|c|c|c|c|c|c|c|c|c|c|c|c|c|c|c|c|c|c|c|c|}
\hline & & & & & & & & & & & & & & & & & & & & \\
\hline Saponaria officinalis & t. & & & & 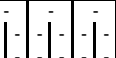 & 1.1 & & j. & & & i & & ${ }^{2}+2>$ & 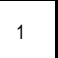 & 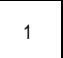 & 2 & Ken & Mediterr & $\mathrm{h}$ & Artemi \\
\hline Sccabiosa ochroleuca & i & & & & & & & & & & 53 & $\left|\begin{array}{|c|c|c|}7 \\
17\end{array}\right|$ & $\left.\right|_{13} ^{9}$ & 12 & ${ }^{36}$ & 50 & $\mathrm{Ha}$ & & $\mathrm{n}$ & Fest-Brom \\
\hline Scleranthus annuus & & & & & & & & & & & i| & i| & & 1 & 1 & 1 & \begin{tabular}{|l|}
$A r$ \\
\end{tabular} & W-Mediterr & $t$ & Stiel medi \\
\hline Scutellaria hastifolia & & & & & & & & & & & $1 j$ & 21 & $i^{2}$ & 2 & 6 & 11 & \begin{tabular}{|l|} 
Ns \\
\end{tabular} & & $\mathrm{h}$ & Moli-Arrh \\
\hline Scilla bifolia L. & & & & & & & & & & & $1 \mid z$ & ${ }^{2} \mid$ & 3 & 2 & 6 & 10 & Ns & & $g$ & |aver-Fage, Rham-Prun \\
\hline Senecio erucifolius L. & 1 & & & & & & & & & & 91 & $\overrightarrow{14}$ & $\left.\right|_{15} ^{17}$ & 16 & 47 & 67 & на & & $\mathrm{n}$ & $\begin{array}{l}\text { Fest-firom, Gail veri, } \\
\text { Aatemi }\end{array}$ \\
\hline $\begin{array}{l}\text { Senecio jacobaea } \\
\text { Waldst. \& Kit. }\end{array}$ & : & $\mathrm{A}: \mathrm{E}$ & & $:$ & & & & & & 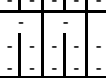 & $\mid \begin{array}{lll}2 & 2 \\
2 & 2 \\
2 & 3 \\
2\end{array}$ & 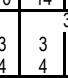 & 表 & 5 & 13 & 17 & Ha & & $\mathrm{n}$ & Fest-Brom, Moli-Arrh \\
\hline $\begin{array}{l}\text { Senecio vernalis } \\
\text { Waldst. \& Kit. } \\
\end{array}$ & i & & & $\vdots$ & & & & 1 & & 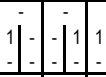 & $\begin{array}{lll} & 4 \\
2 & 2\end{array}$ & $\begin{array}{ll}4 \\
2 \\
2\end{array}$ & 2 & 8 & ${ }^{8}$ & ${ }^{8}$ & $A P$ & & th & Stel medi, Fest vagi \\
\hline $\begin{array}{l}\text { Serratula erucifolia } \\
\text { (L.) Boriss. }\end{array}$ & $-j$ & & & & & & & & & & 55 & | & 52 & 9 & 14 & 18 & Ns & & $\mathrm{h}$ & Fest-Brom \\
\hline Seseli annuum L. & & & & & & & & & & & $j$ & -1 & $3^{3}$ & 3 & ${ }^{4}$ & 6 & Ns & & th & Fest-Brom \\
\hline Seseli tortuosum L. & & & & & & & & & & & $\begin{array}{ll}10 \\
10\end{array}$ & $\mid \begin{array}{ll}144 \\
{ }_{21}\end{array}$ & 竞放 & 13 & 55 & 72 & \begin{tabular}{|l|l|}
$N \mathrm{~s}$ \\
$(\cdots)$
\end{tabular} & & $\mathrm{n}$ & Fest-Brom \\
\hline $\begin{array}{l}\text { Setaria glauca (L.) } \\
\text { P.Beauv. }\end{array}$ & : & & & 11 & $1 / 2$ & & & & & $\begin{array}{l}1 \\
2 \\
2\end{array}$ & $\begin{array}{c}3 \\
20\end{array}$ & & ${ }_{27}^{13}$ & 22 & 59 & ${ }^{84}$ & Ar & |ndian-Malay & t & Stel medi \\
\hline 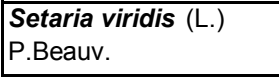 & : & i 121 & & $i$ & & 1 & & & & $\therefore$ & ${ }_{14}^{2} \mid$ & \begin{tabular}{l|l|}
6 & 2 \\
6 & 18 \\
\end{tabular} & $\begin{array}{l}7 \\
7\end{array}$ & 15 & ${ }^{29}$ & 35 & Ar & \begin{tabular}{|l} 
Mediter-raranian- \\
Turanian
\end{tabular} & $\mathrm{t}$ & Stel medi \\
\hline Sideritis montana L. & & & & 1 & & I & & & & $j$ & $j$ & 年 & i & 1 & 1 & 1 & $\mathrm{Ha}$ & & $\mathrm{t}$ & Fest-From, Stel medi \\
\hline Silene nutans $L$. & & & & & & & & & & & i & $1 . i$ & 1. & 1 & 2 & ${ }^{2}$ & Ns & & $\mathrm{h}$ & Trifi-Gera, Quer pube \\
\hline Sinapis arvensis $L$. & & & & & & & & & & & : & 5 & & 6 & ${ }^{10}$ & ${ }^{10}$ & Ar & Mediter-Alantic & ${ }^{t}$ & Stel medi \\
\hline $\begin{array}{l}\mid \begin{array}{l}\text { Sisymbrium } \\
\text { altissimum L. }\end{array} \\
\end{array}$ & & & & & & & & & & & 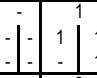 & 1 & 1 & 3 & ${ }^{4}$ & ${ }^{4}$ & Ap & & th & Stiel medi \\
\hline Sisymbr & !. & & & & i: $: 11:$ : & $1: 1$ & & $\mathrm{i}:$ : & ili $\mid i_{2}:$ : & : & 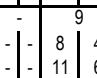 & & $\mid \begin{array}{l}5 \\
7\end{array}$ & 16 & ${ }^{38}$ & 45 & Ar? & $\begin{array}{l}\text { Eurasiaticic- } \\
\text { continant }\end{array}$ & th & Stel meli, Artemi \\
\hline $\begin{array}{l}\text { Sisymbrium polymor- } \\
\text { phum (Murray) Roth }\end{array}$ & $\left.\right|_{2} \mid$ & $2^{2}$ & & $\begin{array}{ll}2 \\
33^{2} \\
31^{2}\end{array}$ & & 更 & & \begin{tabular}{|l|l|l|} 
& 2 \\
2 & 2 \\
2 & 2
\end{tabular} & 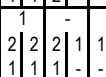 & 12 & \begin{tabular}{c|c}
15 \\
21 \\
21 & 2 \\
20 & 1
\end{tabular} & 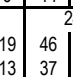 & $\begin{array}{l}4 \\
33 \\
16\end{array}$ & 22 & 88 & 156 & Ns & & th & Fest-Brom \\
\hline \begin{tabular}{|l} 
Solanum dulcamara \\
L.
\end{tabular} & & & & & & 1 & & & & & $i$ & i] i] & i & 1 & ${ }^{2}$ & $2^{2}$ & $\mathrm{Ha}$ & & nf & $\begin{array}{l}\text { Sali urp, Ann glut, Gali- } \\
\text { Urti, Artemi }\end{array}$ \\
\hline Solanum nigrum $\mathrm{L}$. & & & & & & & & & & & $2 \mid \mathrm{c}$ & & $\frac{2}{7}$ & 10 & 13 & 14 & Ar & $\begin{array}{l}\text { Mediter-r- } \\
\text { Atantic? }\end{array}$ & $\mathrm{t}$ & Stiel medi \\
\hline Sonchus arvensis L. & & & & & & & & & & $|:| a \mid$ & i) & il & 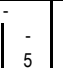 & 5 & 5 & 7 & Arr & 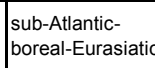 & n & Stel medi \\
\hline \begin{tabular}{|l}
$\begin{array}{l}\text { Sonchus asper (L.) } \\
\text { Hill }\end{array}$ \\
(L).
\end{tabular} & & & & & & & & & & & 3 & & & 6 & 8 & 8 & Ar & Mediterr & $\mathrm{t}$ & Stel medi \\
\hline
\end{tabular}




\begin{tabular}{|c|c|c|c|c|c|c|c|c|c|c|c|c|c|c|c|c|c|c|c|c|c|c|c|c|c|c|c|c|c|c|c|}
\hline & \begin{tabular}{|l|l}
1 & \\
\end{tabular} & 2 & 3 & 4 & 5 & 6 & 7 & \begin{tabular}{|l|l|}
8 & 9 \\
\end{tabular} & \begin{tabular}{l|l|l|}
9 & 10 & 11 \\
\end{tabular} & \begin{tabular}{l|l|l}
12 & 13 \\
\end{tabular} & 14 & 15 & 16 & 17 & 18 & 19 & \begin{tabular}{l|l}
20 & 2 \\
\end{tabular} & \begin{tabular}{l|l|}
21 & 22 \\
\end{tabular} & & \begin{tabular}{|l|l|}
3 & 24 \\
\end{tabular} & 25 & 26 & & 27 & 28 & 29 & 30 & 31 & 32 & 33 & 34 \\
\hline Sonchus oleraceus $\mathrm{L}$. & -1. & & & & & & -1 & $-1-$ & -1. & 101 & & & & & & & 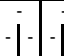 & j. & & & $j$ & & & & 1 & 1 & 1 & Ar & Mediterr & th & Stel medi \\
\hline $\begin{array}{l}\text { Sorghum cfr. } \\
\text { saccharatum (L.) }\end{array}$ & 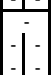 & & & & -1. & 1. & $\because:$ & & : $\left.1 \begin{array}{l}1 \\
1\end{array}\right]$ & & & & & & & & & & & & $\therefore$ & \begin{tabular}{l|l}
1 & $:$ \\
1 & -
\end{tabular} & & $1:$ & 1 & 2 & 2 & $E g$ & S,W-Asiatic & $\mathrm{t}$ & Stel medi \\
\hline $\begin{array}{l}\text { Spiraea hypericifolia } \\
\text { L. }\end{array}$ & i 12 & & & & E & -1 & $\because:$ & & -1 & & & 1 & & & & & (1) & $\perp$ & & & -1 & \begin{tabular}{l|l}
$-i$ & 1 \\
1 & 1 \\
\end{tabular} & & \begin{tabular}{|l|l|}
2 \\
2 \\
\end{tabular} & 1 & 3 & 5 & Ns & & nf & $\begin{array}{l}\text { Fest-Brom, Gali veri, } \\
\text { Rham-Prun }\end{array}$ \\
\hline Stachys annua (L.) L. & $i$ & & & & & & $-1-$ & & $-1-$ & & & & & & & & & & & & $-j$ & & & & 2 & 3 & 3 & Ar & Mediterr & $\mathrm{t}$ & Stel medi \\
\hline Stachys recta L. & \begin{tabular}{|l|l|} 
& - \\
2 & 2 \\
2 & 2 \\
\end{tabular} & & & & & & $\because$ & & \begin{tabular}{|l|c|c|} 
& 1 & 1 \\
1 & 2 & 1 \\
\end{tabular} & & & & & & & & & & & & -1 & \begin{tabular}{|c|c|}
5 & 7 \\
10 & 1 \\
\end{tabular} & & \begin{tabular}{|c|c|}
0 & 9 \\
0 & 14 \\
\end{tabular} & 13 & 36 & 53 & Ns & & $\mathrm{h}$ & Fest-Brom, Gali veri \\
\hline Stellaria graminea L. & 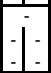 & & 2 & & & & $\therefore$ & $-l_{3}^{3}$ & i & & & & & & & & & & & & -1 & \begin{tabular}{|l|l|}
1 & 6 \\
2 & 7 \\
\end{tabular} & & \begin{tabular}{|l|l|}
12 \\
\end{tabular} & 11 & 17 & 31 & $A p$ & & $\mathrm{~h}$ & $\begin{array}{l}\text { Moli-Arrh, Gali veri, Agro } \\
\text { int-rep }\end{array}$ \\
\hline $\begin{array}{l}\text { Stellaria media (L.) } \\
\text { Vill. } \\
\end{array}$ & -1 & & & & & & . & . & -1 & & & & & & & & & & & & 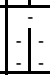 & $\begin{array}{l}\vdots \\
\end{array}$ & & & 1 & 1 & 1 & $A p$ & & th & Stel medi \\
\hline Stipa capillata $\mathrm{L}$. & \begin{tabular}{|l|l|} 
& 1 \\
3 & 3 \\
2 & 2 \\
\end{tabular} & & & & 1] & & & \begin{tabular}{|l|l|} 
& 1 \\
2 & 2 \\
1 & 2 \\
\end{tabular} & \begin{tabular}{|l|l|l|}
-1 & 1 \\
& 2 & 2 \\
& 2 & 1 \\
\end{tabular} & & & & & & & & & & & & -1 & \begin{tabular}{|l|l|}
\multicolumn{1}{|c|}{13} \\
16 & 17 \\
14 & 1 \\
\end{tabular} & & \begin{tabular}{|l|l|}
23 & 38 \\
& 38 \\
\end{tabular} & 17 & 74 & 150 & \begin{tabular}{|c|}
$N s$ \\
$(* * t)$ \\
\end{tabular} & & $\mathrm{h}$ & Fest-Brom \\
\hline Syringa vulgaris $\mathrm{L}$. & $i=$ & & 10 & & -1 & & -1 & $i$ & i. & j. & & & & & & & & & i & & $i$ & $-1:$ & & & 1 & 1 & 2 & Eg & SE European & nf & Robin \\
\hline $\begin{array}{l}\text { Taraxacum erythro- } \\
\text { spermum Andrz. }\end{array}$ & $i$ & & -1 & & & & -1 & $-i$ & $-i$ & & & & & & & & & & & & $i$ & \begin{tabular}{|l|l}
- & 2 \\
2 & 2
\end{tabular} & & \begin{tabular}{l|l}
2 \\
2
\end{tabular} \mid & 4 & 6 & 7 & Ns & & $\mathrm{h}$ & Fest-Brom, Poly-Arte \\
\hline $\begin{array}{l}\text { Taraxacum officinale } \\
\text { Wigg. }\end{array}$ & -12 & & & & -1 & & $\therefore$ & & $\therefore \begin{array}{lll}-1 & -1 \\
2 & 1 & 1 \\
\end{array}$ & & & & & & & & & & & & $-i$ & \begin{tabular}{|l|l|l|}
- & 5 \\
5 & 13 \\
\end{tabular} & & $\mid \begin{array}{c}5 \\
16\end{array}$ & 14 & 25 & 31 & $A p$ & & $\mathrm{~h}$ & Plan majo \\
\hline $\begin{array}{l}\text { Taraxacum serotinum } \\
\text { (Waldst. \& Kit.) Poir. }\end{array}$ & \begin{tabular}{r|r|} 
& 1 \\
2 & 1 \\
\end{tabular} & &. & & - & & $\because 1$ & $\therefore$ & $-1-$ & & & & & & & & & & & & $-\overline{1}$ & \begin{tabular}{|l|l|l}
2 & 2 \\
4 & 3
\end{tabular} & & \begin{tabular}{|l|l|} 
& 2 \\
4
\end{tabular} & 7 & 12 & 17 & $\mathrm{Ha}$ & & $\mathrm{h}$ & Fest-Brom, Poly-Arte \\
\hline $\begin{array}{l}\begin{array}{l}\text { Teucrium } \\
\text { chamaedrys L. }\end{array} \\
\end{array}$ & $\therefore$ & & i. & & & & $\therefore-$ & i & $i$ & & & & & & 11 & 12 & & & & & -1 & \begin{tabular}{|l|l|}
3 & 3 \\
3 & 6 \\
\end{tabular} & & \begin{tabular}{|l|l|} 
& 6 \\
11
\end{tabular} & 6 & 16 & 28 & Ns & & $\mathrm{h}$ & $\begin{array}{l}\text { Fest-Brom, Trif-Gera, } \\
\text { Quer pub-pet }\end{array}$ \\
\hline $\begin{array}{l}\text { Thalictrum minus L. } \\
\text { aggr. }\end{array}$ & \begin{tabular}{|r|r|} 
& 2 \\
2 & 2 \\
\end{tabular} & & $\mid \begin{array}{lll}1 & 2 & - \\
2 & 3 & -\end{array}$ & & \begin{tabular}{|l|l|l|}
2 & 2 \\
2 & 2 \\
\end{tabular} & \begin{tabular}{l|l}
2 \\
3
\end{tabular} & \begin{tabular}{|l|l|} 
& 2 \\
1 & 1 \\
2 & 2 \\
\end{tabular} & & 1 & \begin{tabular}{|l|l|} 
& 1 \\
2 & 3 \\
\end{tabular} & & & & - & & & \begin{tabular}{ll|l}
2 & 2 \\
2 & 2 \\
\end{tabular} & & 1 & & \begin{tabular}{|l|l|}
- & 2 \\
2 & 2 \\
\end{tabular} & \begin{tabular}{|c|c|}
9 & 9 \\
8 & 14 \\
14 & 15 \\
\end{tabular} & & \begin{tabular}{|l|l|}
14 & 25 \\
& 35 \\
\end{tabular} & 15 & 60 & 113 & Ns & & $\mathrm{h}$ & $\begin{array}{l}\text { Fest-Brom, Gali veri, Trif- } \\
\text { Gera }\end{array}$ \\
\hline $\begin{array}{l}\text { Thesium arvense } \\
\text { Horv. }\end{array}$ & $\left|\begin{array}{l}1 \\
1 \\
1\end{array}\right|$ & $\begin{array}{c}- \\
1\end{array} \mid$ & -1 & & & & $1:$ & 11 & -1 & 1 & & & & & & 1 & & & & & \begin{tabular}{l|l}
1 & 1 \\
1
\end{tabular} \mid & \begin{tabular}{|l|l|}
7 & 8 \\
6 & 3 \\
\end{tabular} & $\begin{array}{l}8 \\
6\end{array}$ & \begin{tabular}{|l|l|} 
& 8 \\
3
\end{tabular} & 15 & 25 & 26 & Ns & & $\mathrm{h}$ & Fest-Brom, Poly-Arte \\
\hline $\begin{array}{l}\text { Thesium procum- } \\
\text { bens C.A.Mey. }\end{array}$ & $\therefore$ & $-1:-1$ & : & & 101 & & $\therefore-$ & 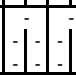 & $-1-$ & $-i_{1}^{1}$ & & & & - & $\begin{array}{lll}1 & 1 \\
& 1 & \\
\end{array}$ & 1 & 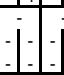 & & 1 & & i. & \begin{tabular}{|l|l|l|}
2 & 3 \\
2 & 2 \\
\end{tabular} & & \begin{tabular}{|l|l|}
3 \\
2 \\
\end{tabular} & 4 & 10 & 10 & Ns & & $\mathrm{h}$ & Fest-Brom \\
\hline Thlaspi arvense $\mathrm{L}$. & -1 & & & & & & . & &. & -1 & & & & & & & & & & & i. & $\dot{4}$ & & $i_{2}$ & 5 & 6 & 6 & Ar & Iranian-Turanian & th & Stel medi \\
\hline $\begin{array}{l}\text { Thymus dimorphus } \\
\text { Klokov \& Des.-Shost. }\end{array}$ & $i$ & & & & & & -1 & & 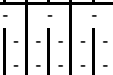 & - & & & & & & \begin{tabular}{|l|l|l|}
2 & \\
2 & 3 & 1 \\
- & - & 2 \\
\end{tabular} & $\begin{array}{l}1 \\
2\end{array}$ & & & & 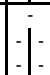 & \begin{tabular}{l|l}
2 & 1 \\
1 &.
\end{tabular} & & 3 & 2 & 5 & 10 & \begin{tabular}{|l|}
$N s$ \\
$(* * * *)$ \\
\end{tabular} & & c & Fest-Brom, Fest vagi \\
\hline $\begin{array}{l}\text { Thymus marscha- } \\
\text { llianus Willd. } \\
\end{array}$ & \begin{tabular}{|l|l|}
1 & 2 \\
1 & 1 \\
\end{tabular} & -12 & 1 & & & & . & . & -1 & $\begin{array}{l}2 \\
1 \\
\end{array}$ & & $\begin{array}{ll}-2 \\
2\end{array}$ & & 2 & & \begin{tabular}{|l|l|}
2 & 2 \\
1 & 2 \\
\end{tabular} & 1. & I & & & 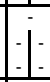 & \begin{tabular}{|l|l|}
8 & 9 \\
8 & 4 \\
\end{tabular} & & \begin{tabular}{|l|c|}
3 & 16 \\
0 & 6 \\
\end{tabular} & 13 & 30 & 47 & Ns & & c & Fest-Brom \\
\hline $\begin{array}{l}\text { Torilis japonica } \\
\text { (Houtt.) DC. }\end{array}$ & 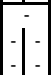 & 1 & 1 & 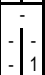 & - & & -1 & $1:$ & -1 & 1 & & & & 2 & & 1 & 1 & & & & 1 & \begin{tabular}{l|l}
- \\
1 & 3
\end{tabular} & & $\dot{4}$ & 3 & 4 & 6 & $A p$ & & th & Artemi \\
\hline $\begin{array}{l}\text { Torilis ucrainica } \\
\text { Spreng. }\end{array}$ & $:-1$ & $\because:$ & & & -1. & & -1 & 1 & $1:$ & -1 & & -1 & & & & -1. & 1 & $i$ & & & 1 & \begin{tabular}{l|l}
$i$ & $i$ \\
\end{tabular} & & $i_{1}$ & 2 & 2 & 2 & $\mathrm{Ha}$ & & $t$ & Artemi \\
\hline $\begin{array}{l}\text { Tragopogon major } \\
\text { Jacq. }\end{array}$ & $\because$ & & 1. & .1. & & i $]_{-1}^{1}$ & & $\mid \begin{array}{ccc}1 & 1 & 1 \\
1 & 1\end{array}$ & $\left|\begin{array}{l|l|l|l|l|}1 & 1 & 1 & 1 & 1 \\
1 & - & 1\end{array}\right|$. & 1. & & 1 & 1 & & & -1 & $\begin{array}{ccc}1 \\
-1\end{array}$ & 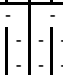 & & & -1 & \begin{tabular}{|l|l}
5 & 4 \\
6 & 1
\end{tabular} & & \begin{tabular}{|l|l}
4 \\
3
\end{tabular} & 10 & 17 & 17 & $A p$ & & $\mathrm{~h}$ & Fest-Brom, Artemi \\
\hline $\begin{array}{l}\text { Tragopogon podoli- } \\
\text { cus (DC.) Artemcz. }\end{array}$ & i & $\therefore-1$ & 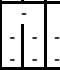 & $1-1$ & & & 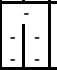 & i. & i. & 1. & & & & & & $\therefore$ & 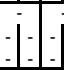 & 1 & 1 & & 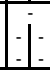 & $: i_{2}$ & & $i_{2}$ & 2 & 2 & 2 & Ns & & $\mathrm{h}$ & Fest-Brom \\
\hline
\end{tabular}




\begin{tabular}{|c|c|c|c|c|c|c|c|c|c|c|c|c|c|c|c|c|c|c|c|c|c|c|c|c|c|c|c|c|c|c|c|c|c|}
\hline & \begin{tabular}{|l|l}
1 & \\
\end{tabular} & 2 & 3 & 4 & 5 & 6 & & 8 & 9 & 10 & 11 & \begin{tabular}{|l|l|}
12 & 13 \\
\end{tabular} & \begin{tabular}{l|l}
13 & 1 \\
\end{tabular} & \begin{tabular}{l|l}
14 & 15 \\
\end{tabular} & 5 & 17 & 18 & 19 & 20 & \begin{tabular}{|l|l|}
21 & 22 \\
\end{tabular} & & \begin{tabular}{l|l|}
23 & 24 \\
\end{tabular} & 25 & 26 & 27 & 7 & 28 & 29 & 30 & 31 & 32 & 33 & 34 \\
\hline Trifolium alpestre $L$. & & & & & & & & -1 & & I & & & T & & T & & & & & & & & $-1-$ & $-1-1$ & & & 1 & 1 & 1 & $\mathrm{Ha}$ & & $\mathrm{h}$ & Trif-Gera, Fest-Brom \\
\hline $\begin{array}{l}\text { Trifolium ambiguum } \\
\text { M.Bieb. }\end{array}$ & 1 & & & & & & & $\therefore:$ & & $\therefore-1$ & & & -1 & & $-1:$ & & & & & & & & $:$ & $:-i_{1}$ & $: 1$ & & 1 & 1 & 1 & Ns & & $\mathrm{h}$ & Trif-Gera, Fest-Brom \\
\hline Trifolium arvense $\mathrm{L}$. & \begin{tabular}{|l|l|} 
& 2 \\
2 & 2 \\
& 1 \\
\end{tabular} & & & & & & & i. & & -1 & & & & & $\begin{array}{rl} & 3 \\
3 & 2 \\
3 & 2 \\
3 & 2 \\
2\end{array}$ & & & & & & & & 1 & \begin{tabular}{|l|l|}
6 & 5 \\
3 & 3 \\
\end{tabular} & \begin{tabular}{l|l|l|}
9 & \\
6 & \\
\end{tabular} & $\begin{array}{l}7 \\
4 \\
\end{array}$ & 8 & 20 & 32 & $A p$ & & $\mathrm{t}$ & \begin{tabular}{|l|}
$\begin{array}{l}\text { Fest-Brom, Fest vagi, Gali } \\
\text { veri, Poly-Arte, Sedo-Scle }\end{array}$ \\
\end{tabular} \\
\hline $\begin{array}{l}\text { Trifolium montanum } \\
\text { L. }\end{array}$ & \begin{tabular}{ll|l}
1 & 2 \\
1 & 2 \\
\end{tabular} & & & & & & & 1. & & -1 & & & 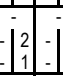 & & -1. & & & & & & & & -1 & \begin{tabular}{|l|l|}
1 & 3 \\
1 & 3 \\
\end{tabular} & \begin{tabular}{l|l}
1 & \\
2 & \\
\end{tabular} & $\begin{array}{l}5 \\
6 \\
\end{array}$ & 3 & 8 & 14 & Ns & & $\mathrm{h}$ & Fest-Brom \\
\hline Trifolium pratense $\mathrm{L}$. & 2 & & & & & & & 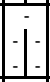 & & & & & & & $i$ & & & & & & & & -1 & \begin{tabular}{|l|l|}
- & - \\
\end{tabular} & & & 2 & 2 & 3 & $\mathrm{Ha}$ & & $\mathrm{h}$ & Moli-Arrh \\
\hline Trifolium repens $\mathrm{L}$. & $\therefore$ & & & & $i$ & & & $\therefore$ & & $\therefore$ & & & 1. & & $\because$ & & & & & $\because$ & $i$ & & e. & $-|i|$ & $: 1$ & i & 1 & 1 & 1 & $A p$ & & $\mathrm{~h}$ & Plan majo \\
\hline $\begin{array}{l}\text { Trigonella } \\
\text { monspeliaca L. }\end{array}$ & $i-1$ & & & & & & & -1. & & & & & & & & & & & & & 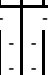 & & $i$ & $i_{1}:-1$ & & & 1 & 1 & 1 & Ns & & $\mathrm{t}$ & Fest-Brom, Poly-Arte \\
\hline Triticum durum Desf. & & & & & & & & & & & & & & & -1 & & & & & & -1 & & -1. & \begin{tabular}{|l|l|}
- & - \\
\end{tabular} & & & 3 & 3 & 4 & $E g$ & SW-Asiatic & $\mathrm{t}$ & Stel medi \\
\hline Ulmus glabra Huds. & 1 & & & & & & & 1. & & & & & & & & & & & & & . & & & & & & 1 & 1 & 1 & Ns & & $\mathrm{mf}$ & Quer-Fage \\
\hline Ulmus minor Mill. & & & & & & -1 & & -1. & & $-1-1$ & & & 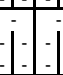 & & $i$ & & & & & & $j$ & & 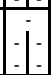 & \begin{tabular}{|l|l|} 
& 1 \\
2 & 3 \\
\end{tabular} & & $\begin{array}{l}1 \\
3\end{array}$ & 4 & 7 & 7 & $\mathrm{Ha}$ & & $\mathrm{mf}$ & $\begin{array}{l}\text { Quer-Fage, Sali purp, } \\
\text { Rham-Prun, Robin }\end{array}$ \\
\hline Ulmus laevis Pall. & & & & & $\therefore$ & $\therefore$ & & 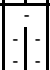 & & 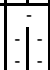 & & & 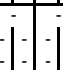 & & $i$ & & 4 & & & $\therefore$ & i & & $i$ & \begin{tabular}{|l|l|} 
& $i$ \\
\end{tabular} & & & 2 & 3 & 4 & Ns & & $\mathrm{mf}$ & Quer-Fage \\
\hline Urtica dioica L. & & & & & & & & i. & & & & & & & + & & & & & & & & -1 & \begin{tabular}{|l|l|}
3 & 3 \\
2 & 8 \\
\end{tabular} & & $\begin{array}{l}4 \\
15 \\
\end{array}$ & 10 & 16 & 27 & $\mathrm{Ha}$ & & $\mathrm{h}$ & Gali-Urti, Robini, Artemi \\
\hline Valeriana officinalis $\mathrm{L}$. & & & & & & $1-1$ & & & & 1. & & & & & & & -1 & & & & -1 & & & 2 & & 2 & 3 & 3 & 3 & Ns & & $\mathrm{g}$ & Moli-Arrh \\
\hline $\begin{array}{l}\text { Verbascum austriacum } \\
\text { Schott ex Roem. \& Schult. }\end{array}$ & -1. & & & & & & & .1 & & & & & 2 & & . & &. & & & 1 & & & -1. & \begin{tabular}{|l|l|}
1 & 1 \\
3 & 2 \\
3 & 2 \\
\end{tabular} & $\begin{array}{l}4 \\
4\end{array} \mid$ & $\begin{array}{l}2 \\
2\end{array}$ & 3 & 11 & 13 & Ns & & $\mathrm{h}$ & Gali veri \\
\hline $\begin{array}{l}\text { Verbascum lychnitis } \\
\text { L. }\end{array}$ & \begin{tabular}{rl|} 
& - \\
\end{tabular} & & & & \begin{tabular}{|l|l|}
2 & 1 \\
2 & 1 \\
\end{tabular} & 2 & & \begin{tabular}{|l|l|}
3 & 2 \\
2 & 2 \\
\end{tabular} & & 2 & & & 2] & & & & $\begin{array}{c}- \\
1\end{array}$ & & & 1 & & & i & \begin{tabular}{|c|c|}
9 & 8 \\
17 & 12 \\
\end{tabular} & & $\begin{array}{c}9 \\
15 \\
\end{array}$ & 17 & 54 & 75 & $\mathrm{Ha}$ & & $\mathrm{h}$ & \begin{tabular}{|l|}
$\begin{array}{l}\text { Gali veri, Fest-Brom, Trif- } \\
\text { Gera, Fest vagi, Artemi }\end{array}$ \\
\end{tabular} \\
\hline $\begin{array}{l}\text { Verbascum } \\
\text { phlomoides L. }\end{array}$ & $i$ & & & & -1 & -1 & & -1 & & -1 & :il & & 1 & & $\mid \begin{array}{ll}1 & 1 \\
2 & 1 \\
2 & 1\end{array}$ & 1 & 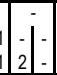 & & $\mid \begin{array}{ll}1 \\
2\end{array}$ & $\begin{array}{lll}1 & - & - \\
1 & 2 \\
\end{array}$ & 1 & & $-i \begin{array}{r}-i \\
2\end{array}$ & \begin{tabular}{|l|l|}
5 & 6 \\
10 & 8 \\
\end{tabular} & $\mid \begin{array}{c}6 \\
15\end{array}$ & $\begin{array}{c}6 \\
10 \\
\end{array}$ & 11 & 32 & 40 & $\mathrm{Ha}$ & & $\mathrm{h}$ & Artemi \\
\hline $\begin{array}{l}\text { Verbascum } \\
\text { phoeniceum } \mathrm{L} .\end{array}$ & \begin{tabular}{|l|l|}
$\begin{array}{l}2 \\
3\end{array}$ & 2 \\
2 & 2 \\
\end{tabular} & & & & & $\left|\begin{array}{l}3 \\
1\end{array}\right|$ & $\begin{array}{l}2 \\
2 \\
\end{array}$ & \begin{tabular}{|l|l|}
3 & 2 \\
2 & 2 \\
\end{tabular} & & \begin{tabular}{|c|c|} 
& 2 \\
3 & 2 \\
2 & 1 \\
\end{tabular} & $\mid \begin{array}{lll}1 & 1 \\
1 & 1 & 1\end{array}$ & & \begin{tabular}{l|l}
2 \\
2 \\
2
\end{tabular} & 1 & 1 & \begin{tabular}{l|l|l}
2 & 2 \\
2 & 1
\end{tabular} & \begin{tabular}{|c|c|c|} 
& 2 \\
2 & 2 \\
1 & 2 & 2 \\
\end{tabular} & & & & & 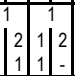 & \begin{tabular}{|l|l|}
\multicolumn{2}{|c|}{} \\
2 & 2 \\
1 & 2 \\
\end{tabular} & \begin{tabular}{|l|l|}
15 & \\
19 & 19 \\
15 & 13 \\
\end{tabular} & & \begin{tabular}{l|}
6 \\
32 \\
17
\end{tabular} & 20 & 81 & 138 & Ns & & $\mathrm{h}$ & Fest-Brom, Fest vagi \\
\hline $\begin{array}{l}\text { Verbascum × ignescens } \\
\text { Tausch }\end{array}$ & $\therefore$ & 1 & & & $\therefore$ & -1 & & $\therefore$ & & 1 & $\because 1$ & & 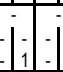 & & 工 & & -1 & & & i. & & & -1 & \begin{tabular}{l|l|}
2 & 2 \\
& 2 \\
\end{tabular} & \begin{tabular}{l|l}
2 \\
\end{tabular} & $\begin{array}{l}2 \\
2 \\
\end{array}$ & 4 & 6 & 6 & Ns & & $\mathrm{h}$ & Fest-Brom, Fest vagi \\
\hline Veronica arvensis $L$. & -1 & & & & & & & 1 & & & & & 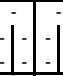 & & i]. & i] & & & & i. & & & - & \begin{tabular}{|l|l|} 
& 1 \\
4 & 3 \\
\end{tabular} & \begin{tabular}{|l|}
3 \\
5
\end{tabular} & $\begin{array}{l}1 \\
4 \\
\end{array}$ & 8 & 10 & 13 & Ar & \begin{tabular}{|l|}
$\begin{array}{l}\text { Mediterr-Iranian- } \\
\text { Turanian }\end{array}$ \\
\end{tabular} & th & $\begin{array}{l}\begin{array}{l}\text { Stel medi, [Fest-Brom], } \\
\text { [Poly-Arte] }\end{array} \\
\end{array}$ \\
\hline Veronica austriaca L. & $\therefore$ & & & & & : & & 1 & & 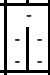 & & $\therefore \begin{array}{rl}-1 & 2 \\
-1 & 2 \\
\end{array}$ & $\begin{array}{l}2 \\
2 \\
2 \\
2 \\
1\end{array}$ & & & & & & & & & & 1 & \begin{tabular}{|l|l|}
1 & 1 \\
3 & 1 \\
\end{tabular} & $\begin{array}{l}2 \\
4 \\
\end{array}$ & $\begin{array}{l}2 \\
1 \\
\end{array}$ & 3 & 7 & 11 & Ns & & c & Fest-Brom \\
\hline $\begin{array}{l}\text { Veronica capsellicarpa } \\
\text { Dubovik }\end{array}$ & 1 & 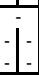 & & -1 & -1 & -1 & -1 & 1 & - & -1 & $\because 1$ & -1.1 & - & i: & -1. & -1 & 1 & & - & 1 & $\begin{array}{l}1 \\
2\end{array}$ & 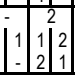 & \begin{tabular}{|l|l|} 
& 1 \\
2 & - \\
\end{tabular} & \begin{tabular}{|l|l|}
3 & 3 \\
3 & 1 \\
\end{tabular} & $\left|\begin{array}{l}4 \\
6\end{array}\right|$ & $\begin{array}{l}4 \\
1 \\
\end{array}$ & 3 & 11 & 17 & Ns & & $\mathrm{h}$ & Fest-Brom \\
\hline \begin{tabular}{l|} 
Veronica chamaedrys \\
L.
\end{tabular} & $\therefore$ & 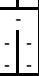 & & $2] 1$ & -1. & -1 & -1 & .1. & -1 & $: 1:$ & -1 & $\begin{array}{lll}- & -1 \\
\end{array}$ & $1 \mid \begin{array}{l}1 \\
1 \\
1\end{array}$ & & 1. & 1 & . & & $: 2$ & $\mid \begin{array}{l}-1 \\
2\end{array}$ & & $\begin{array}{lll}-1 & : & -1 \\
\end{array}$ & i & \begin{tabular}{|l|l|}
- & 3 \\
4 & 5 \\
\end{tabular} & \begin{tabular}{|l|} 
\\
\end{tabular} & $\begin{array}{l}4 \\
6 \\
\end{array}$ & 7 & 12 & 16 & $\mathrm{Ha}$ & & c & Moli-Arrh \\
\hline $\begin{array}{l}\text { Veronica gryniana } \\
\text { Klokov. }\end{array}$ & $i$ & & & & -1 & -1 & 1 & -1 & & -1 & $i$ & \begin{tabular}{rr|r|}
0 & 1 \\
\end{tabular} & $1:-1$ & $1-1$. & -1 & -1 & -1 & & . & 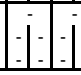 & & $1:-1$. & $\because$ & \begin{tabular}{|l|l|} 
& 1 \\
\end{tabular} & 1. & 1 & 1 & 2 & 2 & Ns & & $\mathrm{h}$ & Fest-Brom \\
\hline
\end{tabular}




\begin{tabular}{|c|c|c|c|c|c|c|c|c|c|c|c|c|c|c|c|c|c|c|c|c|c|c|c|c|c|c|c|c|c|c|c|c|c|c|}
\hline & 1 & 2 & 3 & 4 & 5 & 6 & 7 & 8 & 9 & 10 & 11 & 12 & 13 & 14 & 15 & 16 & $17 \mid$ & 18 & 19 & 20 & \begin{tabular}{|l|l|l}
21 & 22 \\
\end{tabular} & \begin{tabular}{l|l|l|l|l|l|}
22 & 23 \\
\end{tabular} & \begin{tabular}{|l|l|l}
3 & 24 \\
\end{tabular} & 25 & 26 & & 27 & 28 & 29 & 30 & 31 & 32 & 33 & 34 \\
\hline $\begin{array}{l}\text { Veronica hederifolia } \\
\text { L. }\end{array}$ & .. & & & & & & & & & & & & $: 1:$ & & & & & & :D & & & (1.10 & & $:$ & & & & 1 & 1 & 1 & $A p$ & & $\mathrm{t}$ & Stel medi \\
\hline Veronica incana L. & 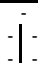 & & & & & & $i$ & & & & & & \begin{tabular}{|l|l|}
2 \\
1 & 2 \\
& \\
\end{tabular} & & & & & & i. & & 10.1 & 1. & & $i=$ & $22_{1}^{2} 2$ & & $\left|\begin{array}{l}5 \\
2\end{array}\right|$ & 2 & 8 & 16 & Ns & & $\mathrm{h}$ & Stel medi, Sedo-Scle \\
\hline Veronica polita Fr. & 1 & & & & & & & & & & & & & & & & & & & & &..$_{1}$ & & 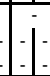 & & & & 1 & 1 & 1 & $A r$ & \begin{tabular}{|l|} 
Mediterr-Iranian- \\
Turanian
\end{tabular} & $\mathrm{t}$ & Stel medi \\
\hline Veronica praecox All. & $1:$ & & & & & & & & - & & & & & & & & & & -1 & & & 1 & & $i$ & $: 11$ & & & 1 & 1 & 1 & $\mathrm{Ha}$ & & th & $\begin{array}{l}\text { Sedo-Scle, Fest vagi, } \\
\text { Fest-Brom, Stel medi }\end{array}$ \\
\hline Veronica prostrata L. &. & & & & & & & & 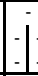 & & & & & - & & & & & & & & -1 & & 2 & \begin{tabular}{|l|l|l}
2 & 1 \\
2 & 3 \\
\end{tabular} & & \begin{tabular}{|l|}
1 \\
5 \\
\end{tabular} & 4 & 8 & 14 & Ns & & c & Fest-Brom \\
\hline $\begin{array}{l}\text { Veronica spicata L. ssp. } \\
\text { barrelieri (Schott) Murb. }\end{array}$ & $\begin{array}{ll}2 & 2 \\
2 & 2 \\
2 & 2\end{array}$ & & & & & & & & 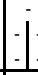 & & & & $2: 1$ & . & & & & & \begin{tabular}{|r}
-1 \\
1
\end{tabular} & & & & & $i$ & & & $\begin{array}{l}8 \\
2 \\
\end{array}$ & 6 & 13 & 21 & Ns & & $\mathrm{h}$ & Fest-Brom, Gali veri \\
\hline Veronica spuria L. & 1. & & & & & & & & 1 & & & & &. & & & & & $\because$ & & & -2 & & $i$ & \begin{tabular}{|l|l|l|}
2 & 2 & 1 \\
2 & 2 \\
\end{tabular} & & $\begin{array}{l}2 \\
4 \\
\end{array}$ & 3 & 9 & 18 & Ns & & $\mathrm{h}$ & Fest-Brom, Trif-Gera \\
\hline Veronica teucrium $\mathrm{L}$. & $1 \%$ & & & & & & & 1.1 & - & & & & & 1. & & & & & $\left.\mid \begin{array}{ll}-1 \\
1\end{array}\right]$ & & & 1.7 & & $i$ & \begin{tabular}{l|l}
1 & 5 \\
3 & 1
\end{tabular} & & \begin{tabular}{|l}
5 \\
1
\end{tabular} & 5 & 10 & 11 & Ns & & ch & Fest-Brom, Moli-Arrh \\
\hline Veronica triphyllos L. & -1. & & & & & & & & & & & & & 5 & & & & & $\mid-1$ & & & & & $i$ & & & & 1 & 1 & 2 & Ar & \begin{tabular}{|l} 
sub-Mediterr-sub \\
Atlantic- \\
European \\
\end{tabular} & $\mathrm{t}$ & Stel medi \\
\hline Veronica verna $L$. &. & & & & & & & & - & & & & & & & & & & \begin{tabular}{|c|c|c|} 
& 2 & \\
2 & 3 \\
2 & 3 & \\
\end{tabular} & & & \begin{tabular}{|l|l}
2 \\
2 \\
2
\end{tabular} & & 22 & \begin{tabular}{|l|l|}
9 & 9 \\
9 & 9 \\
5 & 3 \\
\end{tabular} & $\begin{array}{l}18 \\
10\end{array}$ & $\begin{array}{c}17 \\
6\end{array}$ & 14 & 35 & 68 & $\mathrm{Ha}$ & & $\mathrm{t}$ & $\begin{array}{l}\text { Fest-Brom, Fest vagi, } \\
\text { Poly-Arte }\end{array}$ \\
\hline $\begin{array}{l}\text { Vicia angustifolia } \\
\text { Reichard }\end{array}$ & i & & & & & & & & & & & & & & & & & & & & & $1] 1$ & & -1 & \begin{tabular}{|l|l|}
2 & 7 \\
6 & 7 \\
\end{tabular} & & $\begin{array}{l}9 \\
9\end{array}$ & 11 & 23 & 27 & Ke? & Mediterr? & $\mathrm{t}$ & Stel medi \\
\hline Vicia varia Host & $1:$ & & & & & & & 1.1 & 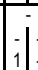 & & & & & & & & & -1 & $1-1$ & & & 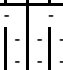 & & $i$ & 1. & & & 1 & 1 & 1 & $\mathrm{Ha}$ & & $\mathrm{t}$ & Stel medi \\
\hline Vicia lathyroides $L$. & $i$ & & & & & & & & & & & & & $:$ & & & & & i. & & -1 & 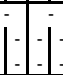 & & $i$ & \begin{tabular}{l|l}
- & 1 \\
- & 1 \\
\end{tabular} & & & 2 & 2 & 2 & Ns & & th & Fest-Brom, Fest vagi \\
\hline Vicia sepium L. & $i$ & & & & & & i. & i & 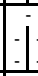 & & & & & 1 & & & 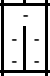 & - & $i$ & & $i$ & $i$ & 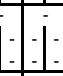 & $-i$ & \begin{tabular}{|l|l}
$i$ & $i$ \\
\end{tabular} & & $i$ & 2 & 2 & 2 & $\mathrm{Ha}$ & & $\mathrm{h}$ & Moli-Arrh \\
\hline Vicia tenuifolia Roth & 1 & & & & & & & 1 & -1 & & & & $\begin{array}{rl}-1 \\
2 & 1 \\
\end{array}$ & - & & & & -1 & -1 & & & $i$ & . & $i$ & \begin{tabular}{l|l}
$i$ & 1 \\
\end{tabular} & & i & 1 & 2 & 3 & $\mathrm{Ha}$ & & gh & Trif-Gera \\
\hline $\begin{array}{l}\text { Vicia tetrasperma (L.) } \\
\text { Schreb. }\end{array}$ & $1:$ & $: 1:-1$ & & & & & $1-1$ & 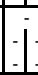 & 1 & & & & $: 1:$ & i & & \begin{tabular}{|l|l|} 
& 2 \\
2 & 2 \\
3 & 2 \\
\end{tabular} & $1-$ & 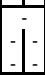 & : & & $\begin{array}{l}1 \\
1\end{array}$ & 1. & L & -1 & \begin{tabular}{|l|l|l|} 
& 2 & \\
3 & 2 \\
4 & 2 \\
\end{tabular} & $\begin{array}{l}5 \\
6\end{array}$ & $\begin{array}{l}3 \\
4 \\
\end{array}$ & 6 & 13 & 21 & Ar & Mediterr & $\mathrm{t}$ & Stel medi, Gali veri \\
\hline Vicia villosa Roth & -1 & & & & & & 2 & i & & & & & & . & & & & 8 & 1.1 & & & $\mid .1$. & & $i$ & & & $\begin{array}{l}1 \\
2\end{array}$ & 3 & 4 & 5 & Ar & Mediterr & th & Stel medi \\
\hline $\begin{array}{l}\text { Vinca herbacea } \\
\text { Waldst. et Kit. }\end{array}$ & $1:$ & $\because \frac{1}{1}$ & -1 & & -1 & & 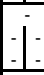 & . & :- & -1 & . & & $\left|\begin{array}{l}1 \\
2\end{array}\right|-$ & - & & 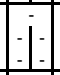 & -1 & $\because$ & -1 & -1 & : & 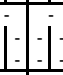 & $\therefore$ & $1:$ & \begin{tabular}{|l|l|}
2 & 2 \\
2 & 1 \\
\end{tabular} & & \begin{tabular}{|l|}
2 \\
1 \\
\end{tabular} & 4 & 8 & 14 & \begin{tabular}{|c|}
$N s$ \\
$\left({ }^{* * * *)}\right.$ \\
\end{tabular} & & $\mathrm{g}$ & Fest-Brom, Trif-Gera \\
\hline $\begin{array}{l}\text { Vincetoxicum } \\
\text { hirundinaria Medik. }\end{array}$ & $1:$ & $\begin{array}{r}-1 \\
1\end{array} \mid \begin{array}{l}1 \\
\end{array}$ & -1 & & & & 1 & . & -1 & & & -1 & i: & $: 1:$ & & -1 & il 2 & -1 & $:-1$ & \begin{tabular}{ll|l|}
1 & 2 \\
2 & 2 \\
\end{tabular} & $\begin{array}{lll} & -1 \\
2 & \end{array}$ & $\mid \begin{array}{lll}1 & -1 \\
2 & -1 & \\
\end{array}$ & 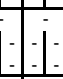 & $1:$ & \begin{tabular}{|l|l|}
1 & 2 \\
4 & 4 \\
\end{tabular} & & \begin{tabular}{|l|}
3 \\
7 \\
\end{tabular} & 5 & 11 & 16 & Ns & & $\mathrm{g}$ & Trii-Gera, Fest-Brom \\
\hline $\begin{array}{l}\text { Viola ambigua Waldst. } \\
\text { \& Kit. }\end{array}$ & $i_{1}^{1}$ & $\because:-1$ &. & & & -1 & -1 & 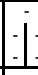 & -1 & -1 & & $1-1$ & $: 1$ &. & & -1 & -1 & -1 & -1 & - & . & 1 & : & $-1:$ & \begin{tabular}{l|l} 
& 1 \\
\end{tabular} & & 1 & 1 & 2 & 2 & Ns & & $\mathrm{h}$ & Fest-Brom \\
\hline Viola arvensis Murray & $1:$ & & & & & & & & & & & & & & & $\left.\mid \begin{array}{l}-1 \\
2\end{array}\right]$ & -1 & & -1 & & 2) & 1. & 1. & \begin{tabular}{l|l}
1 & 1 \\
1 & 1 \\
1 & 2 \\
\end{tabular} & \begin{tabular}{|l|l|l|}
5 & 6 \\
4 & 6 \\
9 & 9 \\
\end{tabular} & & \begin{tabular}{|c|}
9 \\
13
\end{tabular} & 13 & 33 & 48 & Ar & anthropog? & th & Stel medi \\
\hline Viola collina Besser & 1 & \begin{tabular}{|l|l|}
1 & 1 \\
1 & -1 \\
\end{tabular} & D & & & 1. & - & -1 & -1 & -1 & & - 1 & 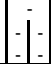 & $\mid-1$ & 1 & . & -1 & -1. & -1 & 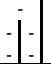 & $: 1$ & t. & $\therefore$ & $i$ & \begin{tabular}{|l|l|}
1 & 1 \\
1 & 1 \\
\end{tabular} & & 1 & 1 & 3 & 3 & Ns & & $\mathrm{h}$ & Fest-Brom, Quer pube \\
\hline Viola hirta L. & -1 & -1. & 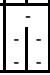 & & & & & & 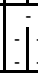 & & & t & -1 & 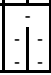 & & & -1 & -1 & -1 & $i$ & 12 & $12: 1$. & 1. & -1 & \begin{tabular}{l|l}
4 & 4 \\
\end{tabular} & & \begin{tabular}{|l|}
2 \\
7 \\
\end{tabular} & 5 & 12 & 18 & Ns & & $\mathrm{g}$ & Trif-Gera, Fest-Brom \\
\hline
\end{tabular}




\begin{tabular}{|c|c|c|c|c|c|c|c|c|c|c|c|c|c|c|c|c|c|c|c|c|c|c|c|c|c|c|c|c|c|c|c|c|c|c|}
\hline & \begin{tabular}{|l|l}
1 \\
\end{tabular} & \begin{tabular}{|l|}
2 \\
\end{tabular} & 3 & \begin{tabular}{|l|}
4 \\
\end{tabular} & 5 & 6 & \begin{tabular}{|l|l|}
7 & \\
\end{tabular} & 8 & 9 & 10 & 11 & 12 & 13 & 14 & 15 & 16 & 17 & 18 & 19 & 20 & 21 & 22 & 23 & 24 & 25 & 26 & 27 & 28 & 29 & 30 & 31 & 32 & 33 & 34 \\
\hline $\begin{array}{l}\text { Viola kitaibeliana } \\
\text { Schult. }\end{array}$ & $1-1$ & $\left|\begin{array}{l}2 \\
2\end{array}\right|$. & -1. & & 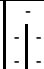 & & & & & 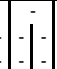 & & & & & & & & & $\because$ & & & & -1. & $\because 1$ & 1 & \begin{tabular}{l|l|}
2 & 2 \\
3 & -
\end{tabular} & \begin{tabular}{|l|l|}
3 & 2 \\
4 & - \\
\end{tabular} & 5 & 8 & 10 & $\mathrm{Ha}$ & & $\mathrm{t}$ & Fest-Brom, Poly-Arte \\
\hline Viola matutina Klokov & $\therefore$ & $\begin{array}{l}1 \\
1 \\
1\end{array}$ & 1 & 2 & $1:$ & & & -1 & & $: 1$ & -1 & & i: & it:- & -1 & $4-1$ & . & & & \begin{tabular}{|l|l|l|}
-2 & 2 \\
2
\end{tabular} &. & & $\begin{array}{|ll|}1 & 2 \\
2 & 2 \\
\end{array}$ & $\begin{array}{ll}1 & 1 \\
-1 & 1 \\
\end{array}$ & 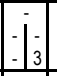 & \begin{tabular}{l|l|}
4 & 5 \\
7 & 8 \\
\end{tabular} & \begin{tabular}{|c|c|}
4 & 8 \\
12 & 16 \\
\end{tabular} & 10 & 28 & 44 & $\mathrm{Ha}$ & & $\mathrm{t}$ & Robin, Sedo-Scle \\
\hline Viola pumila Chaix. & $i$ & $i$ & 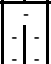 & -1 & $i$ & .9 & $\because i$ & 1 & i & $i$ & -1 & $i$ & $-22^{2}$ & i: & $i$ & $i$ & 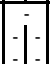 & -1 & i. & $i$ & $i$ & 1.1 .1 & $i$ & 10 & $i$ & $:-11$ & \begin{tabular}{|l|l|}
- & 2 \\
- & -
\end{tabular} & 1 & 1 & 2 & Ns & & $\mathrm{h}$ & Moli-Arrh \\
\hline $\begin{array}{l}\text { Xanthium albinum } \\
\text { (Widder) H.Scholz }\end{array}$ & 1 & & & & & & & & & $i$ & & & & & & & & & 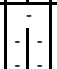 & & & & 1.1. & 1. & $\because$ & & & 1 & 1 & 1 & Ke & North American & $\mathrm{t}$ & Stel medi, [Bident] \\
\hline $\begin{array}{l}\text { Xanthium strumarium } \\
\text { L. }\end{array}$ & $\therefore-1$ & -1 & 1 & $-1:$ & 15 & $-1:$ & . & -1 & 1 & -1 & -1 & -1 & -1 & $1:-$ & -1 & -1 & -1 & -1 & 1. & & $\therefore$ & & $1:$ & $i_{i}$ & $1:$ & \begin{tabular}{l|l|}
1 & 1 \\
\end{tabular} & & 1 & 2 & 2 & Ar & Iranian-Turanian & $\mathrm{t}$ & Stel medi \\
\hline $\begin{array}{l}\text { Xanthoxalis dillenii } \\
\text { (Jacq.) Holub }\end{array}$ & 1 & 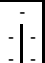 & -1 & 1 & $1:$ & $\because$ & 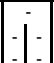 & -1 & t & $i$ & $\because$ & 10 & $\theta$ & $: 1=$ & it & -1 & -1 & 1. & $-1=$ & & $i$ & 1.1 & 1. & 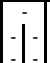 & $\because:-$ & 11 & $j \mid$ & 2 & 2 & 3 & Ke & American & th & Stel medi \\
\hline $\begin{array}{l}\text { Xeranthemum } \\
\text { annuum L. }\end{array}$ & 1 & -1 & -1 & 1 & .1. & 1 & -1 & -1 & -1 & -1 & $\because$ & -1 & -1 & $\because$ & .1 & 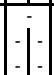 & -1 & $\mid-1$ & 1 & & 1 & & -1. & -1 & -1 & 1 & \begin{tabular}{l|l|} 
& $\vdots$ \\
2 & -1
\end{tabular} & 1 & 1 & 2 & $\mathrm{Ha}$ & & $\mathrm{t}$ & Fest-Brom, Poly-Arte \\
\hline Zea mays L. & $\therefore$ & 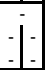 & 1 & $\because-1$. & i: & $\therefore$ & 1. & 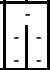 & -1 & i & $\therefore$ & -1 & $\because$ & $\therefore$ & 1 & 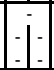 & 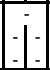 & i. & $\therefore$ & 1 & 1 & -1 & $\therefore$ & 1 & $\because:$ & \begin{tabular}{l|l|}
$i$ & $i$ \\
\end{tabular} & \begin{tabular}{l|l|} 
& $i$ \\
\end{tabular} & 2 & 2 & 2 & $E g$ & South American & $\mathrm{t}$ & Stel medi \\
\hline The number of $\mathrm{T}$ species & 32 & 43 & 30 & 20 & 45 & 52 & 53 & 28 & 16 & 34 & 23 & 38 & 51 & 43 & 20 & 30 & 24 & 19 & 46 & 36 & 15 & 38 & 45 & 61 & 33 & & & & & & & & & \\
\hline The number of Ss species & 17 & 61 & 47 & 37 & 49 & 41 (4) & 39 & 38 & 24 & 47 & 31 & 60 & 70 & 36 & 23 & 41 & 34 & 39 & 63 & 46 & 38 & 48 & 41 & 57 & 52 & & & & & & & & & \\
\hline The number of Sn species & 60 & 43 & 61 & 48 & 56 & 55 & 52 & 50 & 51 & 49 & 44 & 74 & 81 & 62 & \begin{tabular}{|l|}
44 \\
\end{tabular} & 42 & 58 & 50 & 68 & 53 & 45 & 54 & \begin{tabular}{|l|l|}
63 \\
\end{tabular} & 67 & 47 & & & & & & & & & \\
\hline The number of Bs species & 69 & 72 & 60 & 71 & 55 & 55 & 51 & 55 & 47 & 67 & 55 & 72 & 87 & 57 & 46 & 64 & 73 & 54 & 68 & 55 & 75 & 63 & 54 & 67 & 69 & & & & & & & & & \\
\hline The number of Bn species & 90 & 77 & 85 & 66 & 61 & 43 & \begin{tabular}{|l|}
66 \\
\end{tabular} & 57 & 69 & 74 & 60 & 72 & 86 & 67 & $67 \mid$ & 58 & 81 & 68 & 79 & 64 & 72 & 55 & 78 & \begin{tabular}{|l|}
69 \\
\end{tabular} & 57 & & & & & & & & & \\
\hline The total number of species & 117 & 120 & 112 & 118 & 99 & 85 & 90 & 88 & 94 & 104 & 94 & 117 & 159 & 108 & 90 & 92 & 109 & 95 & 121 & 103 & 112 & 114 & 124 & 115 & \begin{tabular}{|l|l|}
107 \\
\end{tabular} & & & & & & & & & \\
\hline Other kurgans from this & zone & & & & & & & & & & & & & & & & & & & & & & & & & & & & & & & & & \\
\hline \begin{tabular}{|l} 
Dianthus andrzejows- \\
skianus (Zapał.) Kulcz.
\end{tabular} & -1 & & 1 & & $1-1$. & & & & & 1 & & & & & & & & & & & & & & & & & & & & & Ns & & $\mathrm{h}$ & Fest-Brom \\
\hline
\end{tabular}

